\author{
Universidade de São Paulo \\ Instituto de Física \\ Departamento de Física Matemática
}

\title{
Extensão da Aproximação de Campo Médio para a Evolução de Sistemas Férmion-Bóson
}

Érica Regina Takano Natti

Tese de Doutoramento

submetida ao Instituto de Física

da Universidade de São Paulo

Banca Examinadora: Prof. Dr. Antônio F. R. de Toledo Piza (USP)

Prof Dra. Kyoko Furuya (UNICAMP)

Prof. Dr. Marcelo Otávio Caminha Gomes (USP)

Profa Dra. Maria Teresa C. dos S. Thomaz (UFF)

Prof. Dr. Salomon S. Mizrahi (UFSCar)

Orientador : Prof. Dr. Antônio Fernando Ribeiro de Toledo Piza

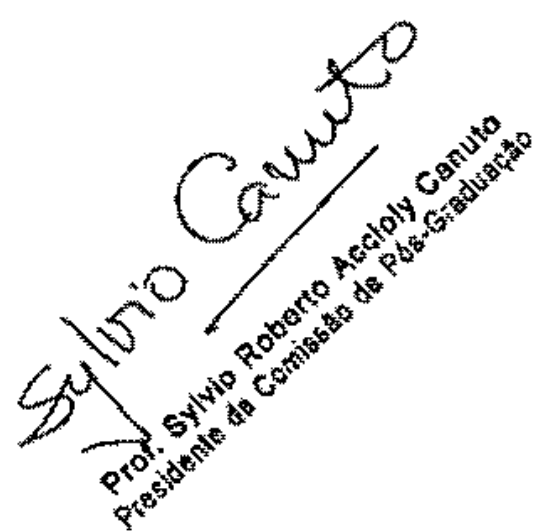

São Paulo

1998

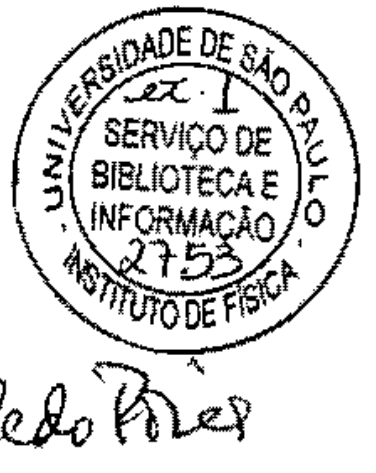

SBI-AFUSP

Apde Thedofices 


\subsection{4 \\ N283e

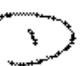 \\ $2 x \cdot 1$}

FICHA CATALOGRÁFICA

Preparada pelo Serviço de Biblioteca e Informação do Instituto de Física da Universidade de Säo Paulo

Natti, Érica Regina Takano

Extensão da Aproximação de Campo Médio para a Evoluçăo de Sistemas Ferrmion-Bóson. São Paulo, 1997.

Tese (Doutoramento) Universidade de São Paulo.

Instituto de Fisica - Departamento de Física Matemática

Área de Concentraçăo: Física Nuclear

Orientador: Prof. Dr. Antonio F. R. de Toledo Piza

Unitermos: 1. Teoria de Muitos Corpos; 2. Tratamento Cinetico; 3. Sistemas Fémion-Bóson; 4. Campo Médio; 5. Técrica de Projeçăo. 
Dedico este trabaho ao Panlo, aos metus pats, José e Teruka $e$ às minhas irmầs, Clá e Lu. 


\section{Agradecimentos}

Ao Prof. Piza, pela orientação, incentivo paciência durante todos estes anos.

Ao Lin, pelas conversas e inúmeras discussós que contribuiram para a conclusão deste trabalho.

Ao Paulo e à minha familia, pelo carinho e apoio em todos os momentos.

Aos meus amigos Ana, Lúcia, Johnny, Magu e Antôno, pelos bons momentos que passamos juntos.

Aos colegas do departamento de físicamatemática, em especial ao Luiz; à Ligia e à Adriana pela colaboraçăo e pela amizade.

A Kelma e Amélia, pela ajuda étimo convivio.

A todos os colegas da USP e da UEL, que de alguma forma contribuiram para este trabalho.

Ao CNPq, pelo apoio financeiro. 
Assim como falham as palavras quando querem exprimir qualquer pensamento,

Assim falham os pensamentos quando querem exprimir qualquer realidade.

Mas, como a realidade pensada nào é a dita mas a pensada,

Assim a mesma dita realidade existe, näo o ser pensada.

Assim tudo o que existe. simplesmente existe.

Fernando Pessoa 


\section{Resumo}

Neste trabalho estudamos a extensão da aproximação de campo médio, dada uma condição inicial, para a evolução temporal de um sistema composto de férmions e bósons que interagem. Para isto usamos uma técnica de projeção dependente do tempo através da qual obtemos equaçōes de movimento do tipo cinético para o conjunto de variáveis dinâmicas de um corpo. Na primeira parte do trabalho aplicamos a técnica para um sistema descrito pelo modelo de Jaynes-Cummings, o qual descreve a interação da matéria, representada por um sistema de dois níveis, com a radiação, representada por um modo normal do campo quantizado. Obtemos a dinâmica de campo médio e a seguir usando a técnica de projeção, calculamos correções à esta descrição de campo médio. Além de ser um modelo exatamente solúvel, o que nos permite comparar nossos resultados com a solução exata, o modelo de Jaynes-Cummings corresponde ao plasma escalar relativístico em zero dimensões espaciais. Na segunda parte deste trabalho estudamos o modelo do plasma escalar relativístico. Esta teoria quântica de campos descreve a interação de campos bosônicos escalares e fermiônicos de spin-1/2 através de uma interação do tipo Yukawa. Para o sistema do plasma escalar relativístico obtemos as equações que descrevem a dinâmica de campo médio e a partir das soluções estacionárias, renormalizamos a teoria. Finalmente, estudamos o regime de pequenas oscilações em torno do equilíbrio, obtendo soluções analíticas para a evolução de nossas variáveis. Analisamos também as condiçōes para existência de estados ligados neste regime. 


\section{Abstract}

In this work we study the extension of the mean-field approximation, given an initial condition, to the time evolution of a fermion-boson system. We use a time-dependent projection where we obtain kinetic-type equations for the set of one-body variables. First, we study the Jaynes-Cummings model which describes the interaction of the matter represented by the two-level system with the radiation represented by the normal mode of the quantized radiation field. We obtain the mean-field dynamics of the system and using the projection technique, we evaluate corrections to this mean-field description. Relevance of the Jaynes-Cummings model stems from the fact that, besides being soluble which possibilities compare our results with the exact solution, it can be seen as corresponding to the relativistic scalar plasma in zero spatial dimensions. Next, we study the relativistic scalar plasma. This quantum field theory describes a system of spin-1/2 fermions interacting through the exchange of scalar particles via a Yukawa-type interaction. In the study of the relativistic scalar plasma, we obtain the mean-field dynamics and from the static solution, we renormalize the theory. Finally, we study the small oscillations regime obtaining analytical solution for one-body variables. We have also examined the condition for the existence of bound-state in this case. 


\section{Conteúdo}

1 Introdução

2 Dinămica Efetiva de Subsistemas Quânticos $\quad 7$

3 Tratamento Cinético para um Sistema Férmion-Bóson: $O$ Modelo de Jaynes-Cummings 14

3.1 Variáveis Gaussianas e sua Dinẫnica Efetivá . . . . . . . . . 14

3.2 Técnica de Projeçăo Dependente do Tempo . . . . . . . . . . . 19

3.3 Aproximação para a Dinâmica de Colisồ $\ldots \ldots \ldots \ldots \ldots \ldots . \ldots 22$

3.4 Resultados e Discussão . . . . . . . . . . . . . . . . 31

4 Equações Cinéticas na Teoria Quântica de Campos 33

4.1 Caracterizaçăo das Variáveis de Interesse $\ldots \ldots \ldots \ldots \ldots \ldots \ldots$

4.2 Dinâmica das Variáveis de Interesse $\ldots \ldots \ldots \ldots \ldots \ldots \ldots, \ldots 3$

4,3 Técnica de Projeçà̃o Dependente do Ternpo ... . . . . . . 46

4.4 Aproximaçăo de Campo Médio . . . . . . . . . . . . . 48

5 Dinâmica Efetiva para o Plasma Escalar Relativístico na Aproximação de Campo Médio $\quad 50$

5.1 Dinâmica de Campo Médio para o Plasma Escalar . . . . . . . . 50

5.2 Renormalizaçăo . . . . . . . . . . . . . 57

6 Linearização da Dinâmica de Campo Médio 67

6.1 Linearização das Equações Cinéticas . . . . . . . . . . . 67

6.2 Soluçio das Equaçốes Cinéticas de Campo Médio Linearizadas . . . . . 72

6.3 Estados Ligados de Quasi-Fémions . . . . . . . . . . . 75

7 Conclusōes e Perspectivas $\quad 79$ 
$\begin{array}{ll}\text { A Cálculo de traços } & \mathbf{8 1}\end{array}$

B Aproximação para $\mathcal{F}^{\prime}(t)$ na Representação de Heisenberg $\quad 85$

C Tabela de Integrais $\quad 89$ 


\section{Capítulo 1}

\section{Introdução}

O estudo de fenômenos de transporte em sistemas de muitos corpos é importante para várias áreas do conhecimento. Estes fenômenos estão associados a processos essencialmente cinéticos, os quais ocorrem em situaçöes descritas como "fora do equilíbrio". Com o objetivo de descrever tais sistemas, em geral, empregant-se técnicas de projeçäo para se derivar as respectivas equaçöes de movimento (equaçóes cinéticas). Embora estas técnicas de projeção sejam extremamente gerais, até o inicio dos anos setenta elas eram somente utilizadas em problemas onde as sistemas estavam em contato com reservatórios [1] ou ainda em problemas onde somente os elementos diagonais da matriz densidade em alguma representação eram desejados [2]. Nestes problemas, os graus de liberdade do reservatorio ou da parte não diagonal da rnatriz densidade eram considerados irrelevantes e formalmente eliminados através de um operador projeção.

Entretanto, há mujtos sistemas dinâmicos acoplados onde nenhum dos subsistemas pode ser considerado como uma parte irrelevante do sistema. Foi neste contexto que Willis e Picard [3] derivaram, utilizando a técnica do operador projeção, equaçōes cinéticas para sistemas dinamicamente acoplados, onde a informação irrelevante não era os graus de liberdade de um dos subsistemas acoplados, mas as correlaçóes entre os subsistemas produzidas pela interaçào. En particular, tal tratamento mostra-se altarnente adequado para sistemas nos quais os subsistemas interagem fortemente. Para estes sistemas a abordagem de Willis e Picard, a qual corresponde em ordem mais baixa a uma aproximaţão do tipo Hartree-Fock Dependente do Tempo (TDHF), è claramente mais apropriada. Outras abordagens, equivalentes a de Willis e Picard, foram também desenvolvidas neste periodo. Assim, a partir de meados dlos anos setenta, vários trabalhos para sistemas quânticos năo relativisticos foram realizados numa aproximaçäo do tjpo TDHF [4] e posteriornente TDHFB [5].

Entretanto, em uma aproximação do tipo TDHF, as partículas do sistema interağen 
somente via campo médio, enquanto as correlaçôes entre as partículas sẫo negligenciadas. Portanto, estes cálculos aplicavam-se por exemplo a sistemas em que as particulas, apesar de interagirem fortemente, formavam um sistema de baixa densidade [6]. Consequentemente, no estudo dos fenômenos de transporte de sistemas de muitos corpos em uma escala de ternpo longa, o qual pode termalizar ou nẫo, as flutuaçôes devido aos efeitos de correlaçăo não podem ser negligenciadas. Portanto, para melhor compreender processos gerais é necessårio estender os cálculos além da aproximaçäo TDHF de modo a incluir correlações de dois corpos. O formalismo necessário para este cálculo foi desenvolvído no final dos anos setenta e início dos anos oitenta e é comumentemente chamado de Aproximação do tipo Hartree-Fock Dependente do Tempo Estendida (ETDHF) $[6,7,8]$. Todos os cálculos ETDHF realizados neste periodo foram para sistemas quânticos não relativisticos.

Simultaneamente, o estudo de sistemas relativisticos de muitos corpos, portanto descritos por uma Teoria Quãntica de Campos, também desenvolvelu-se durante os anos setenta. Estes trabalhos pretendiam descrever a estrutura estacionária de sistemas hadrônicos relativisticos, os quais interagem fortemente, e portanto, o uso de técnicas nãoperturbativas tais como aproximaçōes do tipo Hartree-Fock [0], variacional Gaussiana [10], expansão 1/N [11], função de Wigner covariante [12] ..., eram necessárias.

Os cálculos relativísticos realizados nos anos setenta tinham intereste somente em descrever as propriedades estacionárias dos sistemas nucleares. Contudo, a partir do início dos anos oitenta, a descriçäo de sistemas (fenómenos) quânticos relativisticos fora do equilíbrio dada uma condiçâo inicial ganhou uma importância considerável. Com o advento to cenário do universo inflacionário [13], onde é necessário descrever a evolução temporal de um campo escalar, que se encontra fora do equilibrio térmico com o resto do universo, de um vácuo metaestável para o vácuo estável [14]; e ainda com a entrada em funcionamento dos grandes aceleradores de ions pesados com energias superiores a $0.1 \mathrm{Gev} / \mathrm{A}$, oncle pode-se estudar a matéria nuclear em condiçoes drasticamente fora do equilibrio $\{15,16,17\}$, tornou-se necessário desenvolver o formalismo da Teoria Quảntica de Campos (QF'T) fora do equilibrio.

Assim, com o objetivo de descrever sistemas quânticos relativisticos fora do equilibrio dada uma condição inicial, os quás estăo associados a fenômenos năo-perturbativos tais como quebras de simetrias, geraçäo de massa, transição de fase...., foram desenvolvidas técnicas nào-perturbativas para estudar tais situaçôes no contexto de uma Teoria Quântica de Campos. Em geral estes cálculos foram e săo ainda implementados através de um tratamento cinético auto-consistente baseado em uma aproximaçào de campo médio. ou seja, baseado numa aproximaçäo do tipo TDHF $[17.18,19]$ ou equivalententente baseado no mélodo variacional que utiliza como ansatz um funcional gaussiano para a matriz 
densidade $[14,20,21]$, ou ainda a funçัo de Wigner covariante $[15,22,23]$.

Analogamente ao que ocorreu no caso de sistemas näo relativisticos, a partir do final dos anos oitenta, as técnicas desenvolvidas antertomente, foram adaptadas para descrever a evolução de sistema quântîtos relativísticos fora do equilibrio, dada uma condição inicial, além da aproximaşẫo de campo médio [24, 25].

O objetivo final deste trabalho é descrever a extensão da aproximaçäo de campo médio, dada uma condiçäo inicial, para a evoluçầ de um sistema composto de bósons e férmions que interagem. Para isto utilizaremos uma tecnica desenvolvida anteriormente no contexto da mecânica quântica não relativística por Nemes e Toledo Piza [8]. Esta técuica permite formular a dinâmica de correlaços de um sistema a partir de uma expansão de campo médio, onde recupera-se cs resultados da aproximaçẩo gaussiana em mais baixa ordem, em outras palavras, esta técrica permite incluir e calcular correçóes, devido às correlações dinâmicas, à conhecida aproximação gaussiana. Além disto, a expansão conserva a energia, no caso de sistemas fochados, ordern a ordem [26]. Esta técnica foi recentemente aplicada, no contexto de uma Teoria Quâtica de Campos, a teoria bosönica autointeragente $\lambda \phi^{4}$ em $(1+1)$ dimensões [25]. Lin e Toledo Piza [25] obtiveram que a aproxinação gaussiana falha tanto qualitativamente como quantitativamente na descrição de certos observáveis. As correções colisionais, devido às interaçöes residuais de dois corpos (termos de colisão), corrigem parcialmente os resultados gaussianos. No caso fermionico, Natti e Toledo Piza [19], obtiveram a dinâmica efetiva de um sistema de férmions relativisticos descrito pelo Modelo Quiral de Gross Neveu [11] na aproximação de campo médio.

Motivados pelo sucesso obtido na descriçäo da evoluçào temporal de um sisterra bosônico uniforme autointeragente em $(1 \div 1)$ dimensoes descrito pela teoria $\lambda \phi^{4}$, inim cialmente deslocado do equilibrio, além da aproximaçăo Gaussiana, torna-se interessante estudar, através desta técnica, a evoluçăo temporal de um sistema fora do equilibrio composto de bósons e térmions que interagem. Inicialmente estudaremos um sistema năo relativistico tescrito pelo Modelo de Jaynes-Cummings [27], para em seguida aplicá-la a um sistema relativistico descrito pelo Modelo do Plasma Escalar [28].

Este trabalho o dividido da seguinte forma: No capitulo 2 obternos as equasoes dinamicas que descrevom a evoluço temporal de um sisterna composto de dois subsistemas que interagem. Estas equatoes aplicam-se tanto a sistemas näo relativisticos bem como para sistemats relativisticos. A partir desta equagoes de movimento implementamos uma técnica de projeção [8] e obtemos as equações do tipo cinético desejadas, as quais descrevem avolução de nosso sistema além da aproximaçào de campo médio.

No capitulo 3 aplicamos oformalismo desenvolvido no capítulo 2 a Modelo de aynes. Cummings [27]. Deste modo, descrevemos a evoluçào tempoval, alén da aproximaçäo de campo médio. de um sistema não relativístico composto de dois subsistemas que intera* 
gem, um sistema de dois niveis (subsistema fermiônico) e um modo do campo quantizado (subsistema bosonico). Entre outros resultados, mostramos que a modulação da amplitude das oscilações de Rabi resulta de efeitos de dois corpos (termos de colisăo) [29].

No capitulo 4 adaptamos a técnica de projeção dependente do tempo descrita no capitulo 2 ao estudo de sistema relativisticos. Apresentamos assim, um tratamento näo perturbativo do problema de condiçấ inicial na Teoria Quântica de Campos para um sistema de férmions interagindo com bósons. A partir destas equaçòes discutimos as aproximaçöes conservativas nāo perturbativas que tornam as equaçöes cinéticas obtidas trabalhaveis.

No capitulo 5 aplicamos o formalismo desenvolvido no Capítulo 4 a um sistema relativistico descrito pelo Modelo do Plasma Escalar [28]. Obtemos neste caso a dinâmica efetiva de campo médio (aproximaçâa do tipo TDHFB) de um sistema uniforme relativistico composto de dois subsistemas que ínteragem através de uma interação do tipo Yukawa: um subsistema composto de férmions de spin $1 / 2$ que interagem via troca de bósons escalares (subsisterna bosónico). Neste capítulo fazemos uma renormalizaçầo estática da teoria, ou seja, a partir das equações de equilibrio de nosso sisterna, controlamos as divergências, o que nos permite estudar o estado fundamental do sistema [30].

No capítulo 6 fazemos algumas aplicaçôes para a dinâmica efetiva do Plasma Escalar obtida no capítulo anterior na aproximaçào de campo médio. Primeiramente linearizamos em torno do estado fundamental as equaçōes dinâmicas de campo médio. Em seguida renormalizamos th equaçōes dinănicas linearizadas e obtemos a soluçăo analítica da evoluçào do sisterna neste regime. Finalmente estudamos as condiçôes para a existência de estados ligados no regime de pequenás oscilaçöes en torno do equilíbrio.

No capitulo 7 fazemos um resumo das prineipais conclusöes que o presente estudo permite e traçamos algumas perspectivas de desenvolvimento futuro. Finalmente, alguns detalhes raais técnicos sào apresentados em apêndices. 


\section{Capítulo 2}

\section{Dinâmica Efetiva de Subsistemas Quânticos}

Neste capítulo uma técnica de projeção dependente do tempo desenvolvida por Toledo Piza e Nemes [8] éplitada ao estudo da dinâmica de correlafoóes quânticas entre dois subsistemas. Através desta técnica obtemos equaçóes do tipo cinético que descrevem a evolução temporal do sistema além da aproximaçấ de campo métio.

$O$ uso de técnicas que utilizam operadores de projeção con o objetivo de tratar equaçôes cinéticas nắa é recente. Esta técrica extremamente geral foi muito usada até meados dos anos setenta em problemas onde os sistemas de interesse estavarn em contato com reservatórios, ou ainda, em problemas onde somente os elementos diagonais do operador matriz densidade eram importantes. Nestes problemas, os graus de liberdade do reservatóno ou da parte nāo diagonal do operador densidade eram considerados irrelevantes e formalmente eliminados através de um operador de projeçäo. Entretanto, para sistemas dinâmicos de muitos corpos acoplados conveniente ter urra descriçăo onde cacla parte do sistema (subsistema) é considenda relevante. Willis e Picard [3] derivaram equaçôes cinéticas para um sistema dinamicamente acoplado, onde os graus de liberdade a serem eliminados, năo eram un subsistema ou uma parte do próprio sistema (resetvatório). mas sim as correlações entre os subsistemas produzidas pela interação. Neste formalismo, através de um operador de projeçăo, elimina-se, en ordem mais baixa, as correlacoes entre os subsistemas (conhecidas como termos de colisäo), sendo portanto equivalente ama aproximaça to tipo Hartre-Fock Dependente do Tempo (TDHF).

Por outro lado, para melhor comprecnder os processos irreversíveis é necessário es.

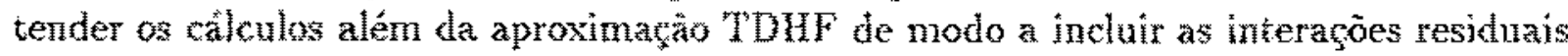
(colisoes de dois corpos). O formalismo necessário para este calculo fó desenvolvido no final dos anos setenta $e$ início dos anos oitenta é comumentemente chamado de Aprom 
ximaça do tipo Hartree-Fock Dependente do Tempo Estendida (ETDHF) [6, 7, 8]. O trabalho desenvolvido por Toledo Piza e Nemes enquadra-se neste cenátio e corresponde a uma aproximaça do tipo Hartree-Fock-Bogoliubov Dependente do Tempo Estendida (ETDHFB).

Passamos agora a descrever esta Técnica de Projecăo considerando um sistema com posto formado de dois subsistemas de diferente natureza: um subsistema fermiònico e um bosônico. Para estudar observaveis associadas a um dos subsistemas de um sistema composto suficiente dispor da densidade reduzida associada ao subsistema de interesse. Tommos como ponto de partida que o sistema descrito por uma densidade completa $F(t)$, cuja evoluçăo temporal é dada pela equação de Liouville von Neumann

$$
i \dot{F}(t)=[H, \mathcal{F}(t)]
$$

onde $H$ é a hamiltoniana total do sistema. Os operadores densidade reduzidos dos subsistemas fermiôntico e bosônico são respectivamente dểnidos por

$$
\begin{aligned}
& \rho(t)=T F F(t) \\
& R(t)=T r_{F} F(t),
\end{aligned}
$$

onde $T r_{3}$ e $T r_{\mathrm{F}}$ denotam traço sobre as densidades bosônicas fermiônicas, respectivamente. Tomando os traços parciais da equaçăo de Liouville von Neumann obtemos equaçós do tipo cinético para as densidades reduzidas,

$$
\begin{aligned}
& \dot{s}(t)=T F_{0}[H, F(t)] \\
& \dot{R}(t)=T_{F}\left[H_{,} \mathcal{F}(t)\right] .
\end{aligned}
$$

Dadas as densidades reduaidas, a densidade

$$
F_{0}(t)=\rho(t) R(t)
$$

referente ao sistema composto, é tal que $T r_{B} F_{0}=p$ e $T r_{r} F_{0}=R$. Como $F_{0}$ e construida inteiramente a partir de $p$ e $R$ sem informaçầ adicional, entầo as correlasōes entre os subsistemas estäo contidas numa densidade $\mathcal{F}^{\prime}(t)$, tambén referente ao sistema composto, tal que 


$$
\mathcal{F}(t)=\mathcal{F}_{0}(t)+\mathcal{F}^{\prime}(t)
$$

O problema com as equuaçôes cinéticas (2.4) e (2.5) é que elas não são fechadas no sentiddo de que a dinâmica é dada en termos do estado completo $\mathcal{F}(t)$. Para controlar essa dificuldade é preciso eserever essas equaçoes em ternos das próprias densidades reduzidas. Dessa forma temos equaçoes fechadas descrevendo a dinâmica dos subsistemas, independentemente de uma referência ac estado $\mathcal{F}(1)$. O problema se reduz entäo a exprimir $\mathcal{F}^{\prime}(t)$ em termos de $\mathcal{F}_{0}(t)$. Para isso Willis e Picard notam que $\mathcal{F}_{0}(t)$ pode ser escrito como

$$
\mathcal{F}_{0}(t)=\mathcal{P}(t) \mathcal{F}(t) \quad \text { com } \quad \mathcal{P}(t)=\mathcal{P}(t) \mathcal{P}(t)
$$

onde $\mathcal{P}(t)$ é um operador de projeção agindo sobre as densidades do sistema composto

$$
p(t)=p(t) T r_{F}+R(t) T r_{B} \cdot-p(t) R(t) T r_{B} T r_{F} .
$$

A densidade $F(t)$ adquire o status de vetor no espaço vetorial de densidades e $\mathcal{P}(t)$ é operador agindo neste espaço. Como as densidades săo operadores no espaço de vetores de estado, os operadores $P(t)$ sào chamados superoperadores eo espaço vetorial de densidades é chamado espaço de Liouville. O produto escalar de dois vetores nesse espaço é definido como

$$
(X, Y)=\operatorname{Tr}\left(X^{+} Y\right)
$$

$O$ projetor adjunto $P^{\dagger}(\xi)$ e o projetor complementar $Q(t)$ são respectivamente definidos por

$$
\begin{aligned}
\left(\mathcal{P}^{\dagger}(t) X, Y\right) & =(X, \mathcal{P}(t) Y) \\
Q(t) & =\mathcal{I}-\mathcal{P}(t)
\end{aligned}
$$

onde I é o operador identidade do espaço de Liouville.

$O$ operador adjunto $P^{\dagger}(t)$ pode ser obtido usando as definigces do produto escalar (2.10) e do operador adjunto (2.11), 


$$
p^{\dagger}=\operatorname{Tr}_{F}(\rho)+\operatorname{Tr}_{B}(R \cdot)-\operatorname{Tr}_{\mathrm{B}} T_{\mathrm{F}}(\rho R \cdot)
$$

Por construçấo $P(t)$ é um operador de projeção dependente do tempo. Portanto, em geral

$$
\frac{d}{d t}[\mathcal{P}(t) \mathcal{F}(t)]=\frac{d \mathcal{P}}{d t} \mathcal{F}(t)+\mathcal{P}(t) \frac{d \mathcal{F}}{d t}
$$

Pode-se no entanto verificar explicitamente que

$$
\frac{d P}{d t} F(t)=0
$$

de modo que, quando age sobre a densidade $F(t)$, o projetor $\mathcal{P}(t)$ é tal que

$$
\frac{d}{d t}[\mathcal{P}(t) \mathcal{F}(t)]=\frac{d \mathcal{F}_{0}}{d t}=\mathcal{P}(t) \frac{d \mathcal{F}}{d t}
$$

Desse modo, a projeçào $\mathcal{P}(t)$ da equação de Liouville-von Neumamn (2.1) resulta em

$$
\begin{aligned}
i \dot{F}_{0}(t) & =P(t)[H, \mathcal{F}(t)] \\
& =P(t) \mathcal{L} \mathcal{F}(t) \\
& =P(t) \mathcal{L}\left[\mathcal{F}_{0}(t)+\mathcal{F}^{\prime}(t)\right]
\end{aligned}
$$

onde foi definido o superoperador que gera deslocamentos da densidade completa,

$$
\mathcal{L} \cdot \equiv[H, \cdot]
$$

chamado Liouvilliano do sistema composto. Nas referências $[\$, 25,20]$ verifica-se que as condiçồ $(2.8)$ e (2.15) tornam a construção de $\mathcal{P}(t)$ única. Por outro lado, devernos ter o cuidado de observar rue a sua construçăo na representação de Schrödinger é dilerente da sua construção na representaçào de Heiseriberg, já que a equaçăo (2.15) é diferente nas duas representaçöes. Na representação de Heisenberg a densidade completa $F$ näo depende do tempo. Neste caso, os operadores de Heisenberg contën todas as complicaçoes da 
dinảmica de muitos corpos. Portanto, o problema crucial é exprimir a dinâmica dos ope radores de maneira que a sua evoluçấo seja dada em ternos das variávels relevantes. Nos capítulos 3 e 4 trabalharemos na representação de Heisenberg de modo que reobteremos as expressōes acima na representaça de Heisenberg.

Dado $\mathcal{P}(t)$ podemos obter uma equaçào para $F^{\prime \prime}(t)$ em termos de $\mathcal{F}_{0}$. Como projeçăo da equaçăo de Liouxille-von Neumann $(2,1)$, a eq. (2.17) contém apenas parte da dinâmica. do sistema composto. Para completála, basta projetar novamente a eq. (2.1) com o projetor complementar a $P$. Com isso, obtemos

$$
i F^{\prime}(t)=Q(t) C\left[\mathcal{F}_{0}(t)+\mathcal{F}^{\prime}(t)\right]
$$

Esta é a equação que permite exprimir em termos de Fo. Podemos resscrevêla como

$$
\left[\frac{d}{d}-\mathcal{Q}(t) \mathcal{L}\right] \mathcal{F}^{t}(t)=\mathcal{Q L F} \mathcal{F}_{0}(t)
$$

cuja soluçäo formal ê dada por

$$
F^{\prime}(t)=G(t, 0) F^{\prime}(0)-i \int_{0}^{t} d t^{\prime} g\left(t, t^{\prime}\right) Q\left(t^{\prime}\right) \mathcal{C} F_{0}\left(t^{\prime}\right)
$$

onde $g\left(t, t^{\prime}\right)$ è a funçâo de Green ordenada no tempo

$$
\mathcal{G}\left(t, t^{\prime}\right)=\mathrm{T} \exp \left[-i \int_{t^{t}}^{t} d \tau \mathcal{Q}(\tau) \mathcal{L}\right]
$$

Desta forma, $F$, e consequentemente $F$, podem ser expressas formalmente em termos de $\mathcal{F}_{0}(1)$ (para $t^{\prime}<$ ). Na realidade, a expressão (2.21) näo é inteiramente fechada em $\mathcal{F}_{0}$ devido ao termo que contém as correlaçóe iniciais $F(0)$. Observamos que o segundo termo da eq. (2.21) reconstrói o restante de $\mathcal{F}^{\prime}(t)$ através de uma integral sobre o passado de $F_{0}(t)$ e tal reconstrução obviamente invätuel para as correlacõos presentes no estado inicial em $t=0$. Por outro lado, é claro também que $\mathcal{F}^{\prime}(0)=0$ significa um estado inicial fatorizado. Deste modo. as equaçóe $(2,4)$ e $(2.5)$ que descrevem a dinâmica efetiva do sistema tornam-se fechadas.

Subatituindo a eq. (2.21) nas eqs. (2.4) e (2.5), utiluando-se a eq. (2.19), obtemos as seguintes equaçoes fechadas para as densidades reduzidas 


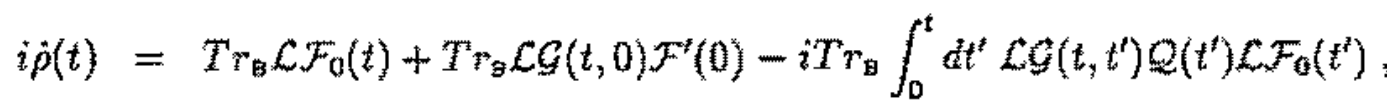

$$
\begin{aligned}
& i \dot{R}(t)=T_{F} \mathcal{L} \mathcal{F}_{0}(t)+T_{F} \mathcal{L} Q(t, 0) \mathcal{F}^{\prime}(0)-i T_{T_{F}} \int_{0}^{t} d t^{t} \mathcal{L} \mathcal{Q}\left(t, t^{t}\right) \mathcal{Q}\left(t^{t}\right) \mathcal{L} \mathcal{F}_{0}\left(t^{t}\right)
\end{aligned}
$$

Os primeiros termos do lado direito dessas duas equaçöes podem ser imediatamente reconhecidos como contribuiçöes de campo médio às dinâmicas efetivas uma vez que $\mathcal{F}_{0}(i)$ é a parte de $\mathcal{F}(i)$ que nảo contém correlaçôes entre os subsistemas. De fato, verificamos que

$$
\begin{aligned}
\operatorname{Tr}_{\mathrm{B}} \mathcal{L} \mathcal{F}_{0}(t) & =\operatorname{Tr}_{\mathrm{B}} L R(t) \rho(t)=\operatorname{Tr}_{\mathrm{B}}(H R \rho-R \rho H) \\
& =\left[\operatorname{Tr}_{\mathrm{B}} H R(t)\right] \rho(t)-\rho(t)\left[\operatorname{Tr}_{\mathrm{B}} H R(t)\right] \\
& \equiv l_{\mathrm{F}}(t) \rho(t)
\end{aligned}
$$

onde foram usadas a propriedade cíclica do traço e as definiçôes

$$
l_{F}(t) \cdot=\left[h_{F}^{(0)}(t),\right\rfloor \quad \text { com } h_{F}^{(0)}(t)=T_{r_{B}} H R(t),
$$

onde $h_{F}^{(0)}(t)$ corresponde à hamiltoniana efetiva de campo médio do subsistema de férmions. Analogamente, para o subsistema de bósons

$$
\operatorname{Tr} C F_{0}(t)=t_{8}(t) R(t) \text {. }
$$

As equaçöes $(2.23)$ e $(2.24)$, com $\mathcal{F}^{\prime}(0)=0$, săo na reatidade equaçỏes do tipo cinéticas acopladas para as densidades reduzidas $\rho(t)$ e R(t $t)$. Elas diferen das equaçōes cinéticas fenomenológicas tasuais pela presença de efeitos de memória (integrais sobre $t^{t}<t$ ) e é por isso conveniente chamá-las equaçö̀es pré-cinéticas.

As equaçồ pré-cinéticas são equaçós exatas que descrevem de forma fechada a dinâmica efetiva dos subsistemas. Notamos entretanto que a complicada dependência temporal da equação (2.21), devido aos efeitos de memória, impede cálculos exatos deste objeto. Um exquema de expansảo sistemática para os efeitos de memória foi discutido nas referencias $\{0,25,26]$. Como vimos, substituir

$$
F(t) \rightarrow \mathcal{F}_{0}(t)
$$


corresponde à aproximaça de campo médio. Nesses trabalhos uma expansäo näo pertubativa em torno do campo médio fol escrita para essas equaţões. As correçōes de correlações ao campo médio em mais baixa ordem correspondem a substituir a evoluçäo temporal dos operadores que ocorrem nas integrais de memória por uma evolução de campo médio dada por

$$
H_{0}=p^{i}(t) H
$$

Consistentemente com esta aproximação, $\mathcal{E}$ substituido em $(2.21)$ e $(2.22)$ por $C_{0}=$ $\left[H_{0}, \cdot\right]$. Desta forma, os efeitos de correlação săo tratados em até segunda ordem em $H$ nas integrais de colisầ resultantes, pois nas integrais de memória em (2.23) e (2.24) há dois Liouvillianos. Una importante caracteristica deste esquema (o qual vale para ordens superiores da expansäo [25]) é que o valor esperado da energía é conservado em todas as ordens, ou seja:

$$
\frac{\partial}{\partial t}(n)=0
$$

onde

$$
\langle H\rangle=T r H F_{0}(t)+T r H F^{\prime}(t)
$$

Nos capitulos seguintes aplicaremos as expressões gerais obtidas acima para tratar primeiramente um sistema fétmion-boson näo relativistico descrito pela hamiltoniana de Jaynes Cummings e posteriomente um sistema uniforme relativistico composto de férmions e bosons escalares interagindo atraves de uma interaçäo do tipo Yukawa. 


\section{Capítulo 3}

\section{Tratamento Cinético para um Sistema Férmion-Bóson: O Modelo de Jaynes-Cummings}

Neste capítulo a técnica de projeçăo dependente do tempo descrita anteriomente é aplicada zo estudo da dinâmica de correlaçôs quânticas de um sistema de férmionz interagindo com bósons, descrito pela Hamiltoniana de Jaynes-Cummings. Atraves desta tecnica obtemos equacoes do tipo cinético que destrevem a evolução temporal do sistema. A partir desta equaçoes discutimos as aproximaços näoperiurbativas nectsatrias para que as equaçes se tornem trabalháveis. A escolha do Modelo de Jaynes-Cummings baseia-se no fato de aue, alén de ser um modelo solúvel, corresponde ao modelo relativistico do plasma escalar em zero dimensões espaciais. $O$ estudo de modelos solúveis fornece um claro entendimento dos fenomenos físicos envolvidos, além de ser útil no controle das várias aproximaçöes necessárias para o tratamento de modelos mais realísticos. Este capítulo corresponde ao trabalho da referência [29].

\subsection{Variáveis Gaussianas e sua Dinâmica Efetiva}

O Modelo de Jaynes Cummings (JCM) [27] descreve a interaçũo de dois subsistemas de diferente naturesa: um sistema de dois niveis (sistema fermionico) e um modo do campo quantizado (sistema bosonico). A Hamiltoniana exatamente solúvel é dada por

$$
H=\frac{c}{2}\left(a_{1}^{\dagger} a_{1}-a_{-1}^{\dagger} a_{-1}\right)+\omega b^{\dagger} b+\lambda\left(a_{1}^{\dagger} a_{-1} b+a_{-1}^{\dagger} a_{1} b^{\dagger}\right)
$$


onde os operadores de aniquilação e criaçäo $\left[b_{\xi}, b^{t}\right]=1$ e os operadores fermionicos $a_{\lambda}$ e $a_{\lambda}^{\dagger}$ satisfazem as relaçóes de anticomutaçăo $\left\{a_{\lambda}, a_{\lambda^{\prime}}^{\dagger}\right\}=\delta_{\lambda_{1}^{\prime} \lambda}$, enquanto os demais comutadores anticomutadores se anulam. Este mo delo descreve a interaçầo da radiação com a matéria (näo relativisticamente). A radiação é representada por um modo normal do campo quantizado e a matéria por um sistema de dois niveis. A interação entre os dois subsistemas é tal que a emissäo ou absorçäo de um quantum de energia pelo campo provoca o abaixamento ou levantamento do nível de energia do sistema de dois niveis. $O$ parâmetro $\varepsilon$ e a frequência natural de transição do sistema de dois niveis, to é a frequência do modo nornal do campo quantizado e $\lambda \dot{\mathrm{e}}$ a constante de acoplamento entre os dois subsistemas.

A solubilidade do modelo vern do fato de que o termo de interação da hamiltoniana é escrito na chamada Rotating Wave Approximation [34], que consiste em ignorar os termos "anti-ressonantes" envolvendo $a_{1}^{*} a_{-1} b^{\dagger}$ e seu conjugado hermitiano.

A idéa geral do nosso tratamento fobter a cvolucta temporal das variatveis de um corpo, ou seja, valores esperados doz operadores e suas combinaçós bilineares ou quadráticas, as quais chamamos varuáveis gaussianas. 0 estado do sistema é dado em termos de uma ruatriz densidade $\mathcal{F}$ ra Representação de Heisenberg. $\mathcal{F}$ é hermitiana, independente do tempo e tem traço unitário. Uma das variáveis de interesse é o valor esperado do operador bosônico

$$
(b)=B(1)=\operatorname{Tr}_{g F} b F
$$

e seu complexo conjugado. Como contém densidades de bósons efémions, $T r_{\mathrm{as}}$ denota traço sobre ambas as densidades. Definimos o operador deslocado

$$
d(t)=b(t)-B(t)
$$

cujo valor esperado em $\mathcal{F}$ se anula

Os valores esperados das formas bilineares dos operadores bosonicos sào dados pela matriz densidade estendida de um bóson [31]

$$
R_{B}=\left[\begin{array}{cc}
R_{B} & \Pi_{3} \\
\Pi_{B}^{*} & 1+R_{B}
\end{array}\right]=\left[\begin{array}{cc}
\left\langle d^{\dagger}(t) d(t)\right\rangle & \langle d(t) d(t)\rangle \\
\left\langle d^{\dagger}(t) d^{\dagger}(t)\right\rangle & \left\langle d(t) d^{\dagger}(t)\right\rangle
\end{array}\right]
$$

onde a matriz densidade normal de um bóson $R_{3}$ é hermitintua e a matriz densidade do eruparehamento de um boson $\Pi_{\mathrm{z}}$ e simétrica.

Para o sistema fermionico a matriz densidade estendida de um férmion [3]] 


$$
\mathcal{R}_{\mathrm{F}}=\left[\begin{array}{cc}
R_{\mathrm{F}} & \Pi_{\mathrm{F}} \\
-\Pi_{\mathrm{F}}^{*} & 1-R_{\mathrm{F}}
\end{array}\right]=\left[\begin{array}{cc}
\left\langle a_{\lambda^{\prime}}^{\dagger}(t) a_{\lambda}(t)\right\rangle & \left\langle a_{\lambda^{\prime}}(t) a_{\lambda}(t)\right\rangle \\
\left\langle a_{\lambda^{\prime}}^{\dagger}(t) a_{\lambda}^{\dagger}(t)\right\rangle & \left\langle a_{\lambda^{\prime}}(t) a_{\lambda}^{\dagger}(t)\right\rangle
\end{array}\right],
$$

onde a matriz hermitiana $R_{F}$ e a matriz antissimétrica $\Pi_{F}$ são respectivamente as matrizes densidade nornal e de emparelhanento de um férmion.

Quando escritas na base de partículas, $\mathcal{R}_{\mathrm{B}}$ e $\mathcal{R}_{\mathrm{F}}$ são näo diagonais pois existem em geral densidades de emparelharuento. Mas $\mathcal{R}_{\ni}$ e $\mathcal{R}_{F}$ podem ser diagonalizadas através de uma transformaçăo de Bogoliubov, de modo que na base de quasi-particulas as densidades de emparelharnento säo nulas. Portanto, para tratar as correlaçôes de emparelhamento en nosso formalismo empregaremos a técnica de Hartree-Fock-Bogolinbov também conhecida como Time Dependent Hartree-Fock-Bogoliubov (TDHFB) [5]. Ela consiste em diagonalizar a matriz densidade estendida com o propósito de tratar as correlaçóes de emparelhamento em termos dos orbitais naturais. Fm seguida toma-se as derivadas temporais das variáveis gaussianas e utilizando-se a equação de Heisenberg obtém-se a dirâmica efetiva destas.

No caso dos bósons definimos os operadores de quasi-bósons como

$$
\begin{aligned}
& \beta(t)=x^{*}(t)\left[b(t)-B^{\prime}(t)\right]+y^{*}(t)\left[b^{\dagger}(t)-B^{*}(t)\right] \\
& B^{\dagger}(t)=x(t)\left[b^{\dagger}(t)-B^{*}(t)\right]+y(t)[b(t)-B(t)]
\end{aligned}
$$

tal que $(\beta \beta)=\left(\beta^{\dagger} \beta^{\dagger}\right)=0$. A preservação das relaçöes de comutaçăo requer ainda que os coeficientes da transformação $x$ e $y$ sejam tais que $|x|^{2}-\left|y^{2}\right|^{2}=1$. Assim, as densidades $\left\langle d^{\dagger} d\right\rangle,\left\langle d d^{\dagger}\right\rangle,\left\langle d^{\dagger} d^{\dagger}\right\rangle$ e $\langle d d\rangle$ podem ser paranetrizadas em termos de $x, y$ e $v=\left\langle\beta^{\dagger} \beta\right\rangle$.

Os coeficientes $x$ e $y$ da transformaçào de Bogoliubov são determinados pela equação secular que diagonaliza $R_{B}$, ou seja,

$$
G R_{\mathrm{B}} X_{\mathrm{B}}=\mathcal{X}_{\mathrm{B}} G Q_{\mathrm{B}}
$$

onde

$$
\mathcal{X}_{\mathrm{B}}=\left[\begin{array}{cc}
x(t) & y^{*}(t) \\
y(t) & x^{*}(t)
\end{array}\right] \quad \mathrm{G}=\left[\begin{array}{cc}
1 & 0 \\
0 & -1
\end{array}\right] \quad Q_{\mathrm{B}}=\left[\begin{array}{cc}
\nu(t) & 0 \\
0 & 1+\nu(t)
\end{array}\right]
$$


Sendo $Q_{a}$ a matriz densidade estendida de um bóson na base de quasi-partículas, o autovalor $\nu$ pode ser interpretado como o número de ocupação dos bósons deslocados para o orbital natural emparelhado descrito por $\mathcal{X}_{8}$. Uma vez que a transformação de Bogoliubov é canônica, podemos verificar que $\mathcal{X}_{B}$ satisfaz as relaçöos de ortogonalidade e completeza

$$
\mathcal{X}_{3}^{\dagger} G X_{\mathrm{B}}=X_{\mathrm{B}} G X_{\mathrm{B}}^{\dagger}=G .
$$

Analogamente, para os operadores fermiônicos definimos a trantsformação de Bogoliuboy

$$
\left[\begin{array}{c}
\alpha_{1}(t) \\
\alpha_{-1}(t) \\
\alpha_{1}^{\dagger}(t) \\
\alpha_{-1}^{\dagger}(t)
\end{array}\right]=\left[\begin{array}{cc}
X_{F}^{*} & 0 \\
0 & X_{F}
\end{array}\right]\left[\begin{array}{c}
a_{1}(t) \\
a_{-1}(t) \\
a_{1}^{\dagger}(t) \\
a_{-1}^{\dagger}(t)
\end{array}\right]
$$

onde

$$
X_{\mathrm{F}}=\left[\begin{array}{cc}
u(t) & -v(t) \\
v^{*}(t) & u^{n}(t)
\end{array}\right] .
$$

Por construçào temos que $\left\langle\alpha_{\lambda}(t) \alpha_{\lambda}(t)\right\rangle=\left\langle\alpha_{\lambda}^{\dagger}(t) \alpha_{\lambda}^{\dagger}(t)\right\rangle=0$, enquanto as densidades fermionicas $\left\langle a_{\lambda}^{\dagger}(t) a_{\lambda}(t)\right\rangle,\left(a_{\lambda}(t) a_{\lambda}^{\dagger}(t)\right\},\left\langle a_{\lambda^{\prime}}^{\dagger}(t) a_{\lambda}^{\dagger}(t)\right)$ e $\left\langle a_{\lambda^{\prime}}(t) a_{\lambda}(t)\right\rangle$ são parametrizadas em termos de $u, v$ e $p_{\lambda}=\left\{\alpha_{\lambda}^{\dagger} \alpha_{\lambda}\right\rangle$ onde $\lambda=-1,1$. Como no caso dos bósons, temos um problema de autovalor

$$
\mathcal{X}_{\mathrm{F}}^{\dagger} \boldsymbol{R}_{\mathrm{F}} \mathcal{X}_{\mathrm{F}}=Q_{\mathrm{F}}
$$

onde $\mathcal{X}_{F}$ é a tranformaçăo definida em $(3.9)$ e $(3.10)$

$$
X_{F}=\left[\begin{array}{cc}
X_{\xi}^{*} & 0 \\
0 & x_{F}^{*}
\end{array}\right] \text { e } X_{F}^{*}=\left[\begin{array}{cc}
X_{F}^{T} & 0 \\
0 & X_{F}^{*}
\end{array}\right]
$$

satisfazendo as equaçòes de unitariedade

$$
\mathcal{X}_{F}^{\dagger} \mathcal{X}_{F}=\mathcal{X}_{F} \mathcal{X}_{F}^{\dagger}=I_{4}
$$


A matriz $Q_{8}$ é a matriz densidade estendida de um férmion na base de quasi-particulas

$$
Q_{\mathrm{F}}=\left[\begin{array}{cc}
p_{\lambda}(t) & 0 \\
0 & 1-p_{\lambda}(t)
\end{array}\right]=\left[\begin{array}{cc}
\left\langle\alpha_{\lambda^{\prime}}^{\dagger}(t) \alpha_{\lambda}(t)\right\rangle & 0 \\
0 & \left\langle\alpha_{\lambda^{\prime}}(t) \alpha_{\lambda}^{\dagger}(t)\right\rangle
\end{array}\right] .
$$

onde $p_{\lambda}$ é a matriz densidade de um férmion na base de quasi-partículas. Nesta base $p_{*}$ é diagonal com autovalores $p_{1}$ e $p_{-1}$ que correspondem aos números de ocupaçäo dos orbitais naturais.

O próximo passo é obter a evolução temporal das variáveis gaussianas. Começamos com o subsistema bosônica. Para $B(t)$ encontra-se imediatamente

$$
i \dot{B}=T r_{B F}[b, H] \mathcal{F}=x T r_{B F}[B, H] \mathcal{F}-y^{* T r_{B F}}\left[P^{\dagger}, H\right] \mathcal{F}
$$

onde $H$ é a hamiltoniana dada por (3.1).

Para tratar as demais densidades inicialmente reescrevemos a equaçăo de autovalor (3.6), usando (3.8), como

$$
\mathcal{X}_{\mathrm{B}}^{\dagger} \mathcal{R}_{\mathrm{B}} \mathcal{X}_{\mathrm{B}}=Q_{\mathrm{B}}
$$

Derivando em relaçào à tempo temos

$$
\begin{aligned}
\mathcal{X}_{\mathrm{B}}^{\dagger} \dot{R}_{\mathrm{B}} \mathcal{X}_{\mathrm{z}} & =\dot{Q}_{\mathrm{B}}-\dot{\mathcal{X}}_{\mathrm{B}}^{\dagger} \mathcal{R}_{\mathrm{B}} \mathcal{X}_{\mathrm{B}}-\dot{X}_{\mathrm{B}}^{\dagger} \mathcal{R}_{\mathrm{B}} \dot{\mathcal{X}}_{\mathrm{B}} \\
& =\dot{Q}_{\mathrm{B}}-\dot{X}_{\mathrm{B}}^{\dagger} G \mathcal{X}_{\mathrm{B}} G Q_{\mathrm{B}}-Q_{\mathrm{B}} G \mathcal{X}_{\mathrm{B}}^{\dagger} G \dot{X}_{\mathrm{B}}
\end{aligned}
$$

O lado esquerdo desta equação pode ser calculado usando a equaçẫo de Heisenberg

$$
i \mathcal{X}_{\mathrm{B}}^{\mathrm{t}} \dot{\mathcal{R}}_{\mathrm{a}} \mathcal{X}_{\mathrm{B}}=\left(\begin{array}{cc}
\operatorname{Tr}_{\mathrm{BP}}\left[\beta^{\dagger} \beta, H\right] \mathcal{F} & \operatorname{Tr}_{\mathrm{BF}}[\beta \beta, H] \mathcal{F} \\
\operatorname{Tr}_{\mathrm{BF}}\left[\beta^{\dagger} \beta^{\dagger}, H\right] \mathcal{F} & \operatorname{Tr}_{\mathrm{BF}}\left[\beta \beta^{\dagger}, H\right] \mathcal{F}
\end{array}\right) .
$$

O lado direito da equação (3.17) pode, por outro lado, ser reestrito usando (3.7). Igualando o resultado à eq. (3.18), temos equaçōes que descrevem a evoluçâo temporal dos coeficientes a e y da transformação de Bogoliubov e da ocupaçào de quasi-bósons $\nu$, 


$$
\begin{aligned}
\dot{\nu} & =\operatorname{Tr}_{\mathrm{BF}}\left[\beta^{\dagger} \beta, H\right] \mathcal{F} \\
i(1+2 y)\left(\dot{x}^{*} y^{*}-x^{*} y^{*}\right) & =\operatorname{Tr}_{\mathrm{Bx}}[\beta \beta, H] \mathcal{F} .
\end{aligned}
$$

Ou seja, a partir desse sistema de equaços diferenciais acopladas temos a evoluçâno temporal das variáveis gaussianas bosonticas.

Temos agora que obter equações análoggas para as variaveis gaussinanas fermiônicas. Derivando em relação ao tempo a equação de autovalor (3.11) e usando a equação de unitariedade (3.13) obtemos

$$
\mathcal{X}_{\mathrm{F}} \dot{\mathcal{R}}_{\mathrm{F}} \mathcal{X}_{\mathrm{F}}=\dot{Q}_{\mathrm{F}}-\dot{\mathcal{X}}_{\mathrm{F}}^{\dagger} \mathcal{X}_{\mathrm{F}} Q_{\mathrm{F}}-Q_{\mathrm{F}} \mathcal{X}_{\mathrm{F}}^{\dagger} \dot{\mathcal{X}}_{\mathrm{F}}
$$

Substituindo (3.12) e (3.14) e usando a equaçăo de unitariedade (3.13) podemos calcular o lado direito da eq. (3.21). Para calcular o lado esquerdo usamos a equação de movimento de Heisenberg. Obtemos

$$
\begin{aligned}
\dot{p}_{\lambda} & =\operatorname{Tr}_{\mathrm{BF}}\left[\alpha_{\lambda}^{\dagger} \alpha_{\lambda}, H\right] \mathcal{F}, \quad \lambda=-1,1 \\
i\left(p_{1}-p_{-1}\right)\left(\dot{u}^{*} v^{*}-\dot{v}^{*} u^{*}\right) & =\operatorname{Tr}_{\mathrm{BF}}\left[\alpha_{-1}^{\dagger} \alpha_{1}, H\right] \mathcal{F}
\end{aligned}
$$

qque descrevem a dinâmica das variảveis fermiônicas.

\subsection{Técnica de Projeção Dependente do Tempo}

As eqs. (3.15), (3.19), (3.20), (3.22) e (3.22) formam uni sistema de equaçöes diferenciais acopladlas que descrevem a evoluçâo temporal de nossas variáveis bosônicas e fermiônicas. Estas equaçồes nâo são contudo fechadas em nossas variáveis de um corpo pois, dada a matriz densidade completa $\mathcal{F}$, elas dependem da hamiltoniana do sistema que por sua vez contêm inleraşôes de dois corpos [termo de interaçào entre os subsistemas - veja eq. (3.1)]; neste caso, as equa cöes que descrevem a dinâmica de $R$ envolverăo também densidades de dois corpos. 
Una maneira de controlar estas dificuldades é através da técnica de projeção dependente do tempo desenvolvida por Toledo Piza e Nemes [8]. Como já foi dito no capitulo anterior, esta técnica consiste em decompor a matriz densidade completa de muitos corpos $F$ em duas partes,

$$
\begin{aligned}
\mathcal{F} & =\mathcal{F}_{0}(t)+\mathcal{F}^{\prime}(t) \\
& \equiv \mathcal{F}_{0}^{\mathrm{a}} \mathcal{F}_{0}^{\mathrm{g}}+\mathcal{F}^{\prime}(t)
\end{aligned}
$$

onde a forma fatorizada de $\mathcal{F}_{0}(t)$ corresponde ao que chamarnos de dupla aproximaçäo de campo médio descrevendo os aspectos de urn corpo da densidade completa. F. As densidades $\mathcal{F}_{0}^{B}$ e $\mathcal{F}_{0}^{F}$ são densidades gaussianas escritas na forma de uma exponencial de una expressão hermitiana bilinear nos operadores de criaçăo/aniquilação bosônicos $\mathrm{e}$ fermiönicos, respectivamente. Quando explicitamente normalizadas a traço unitário, elas săo dadas em termos dos operadores transformados introduzidos em (3.5) e (3.9) como $[8,32]$

$$
\begin{aligned}
& \mathcal{F}_{0}^{F}=\prod_{\lambda}\left[p_{\lambda} \alpha_{\lambda}^{\dagger} \alpha_{\lambda}+\left(1-p_{3}\right) \alpha_{\lambda} \alpha_{\lambda}^{\dagger}\right] \\
& \mathcal{F}_{0}^{*}=\frac{1}{1+\nu}\left(\frac{y}{1+\nu}\right)^{\beta^{\prime} \beta} .
\end{aligned}
$$

No Apêndice $A$ encontram-se os cákculos dos traços utilizudos na aproximaçào de campo médio, nos quais verificamos que dada a densidade $\mathcal{F}_{0}=\mathcal{F}_{0} \mathcal{F}_{0}^{*}$, temos

$$
\begin{aligned}
& \operatorname{Tr}_{\mathrm{Bg}}\left(\alpha_{\mathrm{a}} \mathcal{F}_{0}\right)=T \mathrm{~T}_{\mathrm{QF}}\left(\alpha_{a}^{\dagger} \mathcal{F}_{0}\right)=0 \\
& \operatorname{Tr}_{B F}\left(\alpha_{a} \alpha_{b} \mathcal{F}_{0}\right)=T T_{B F}\left(\alpha_{\alpha}^{\dagger} \alpha_{b}^{\dagger} \mathcal{F}_{0}\right)=0 \\
& \operatorname{Tr}_{\mathrm{ar}}\left(\alpha_{a}^{\dagger} \alpha_{b} \mathcal{F}_{0}\right)=p_{a} \delta_{a b} \\
& T r_{B F}\left(\alpha_{a} \alpha_{b}^{t} F_{0}\right)=\left(1-p_{B}\right) \hat{\delta}_{\mathrm{g}} \\
& \operatorname{Tr}_{\mathrm{BF}}\left(\beta \mathcal{F}_{0}\right)=\operatorname{Tr}_{\mathrm{BF}}\left(\beta^{\dagger} \mathcal{F}_{0}\right)=0 \\
& T r_{Q F}\left(\beta 3 F_{0}\right)=T_{r_{B F}}\left(\beta^{\dagger} \beta^{\dagger} \mathcal{F}_{0}\right)=0 \\
& T^{T}\left(3^{\dagger} \beta \mathcal{F}_{0}\right)=v \\
& T r_{B F}\left(33^{\dagger} \mathcal{F}_{0}\right)=(1+v)
\end{aligned}
$$


A densidade $F_{0}$ descreve completamente os aspectos de um corpo da matrit densidade completa $F$. A densidade $F^{\prime}(1)$ e consequentemente a parte que descreve as correlacoes da densidade completa F. Devido a forma especial usada para Fo $F^{2}$, a densidade F" contém, en geral, correlaçōes de dois tipos: correlações inter-subsistemas (bóson-fémion)

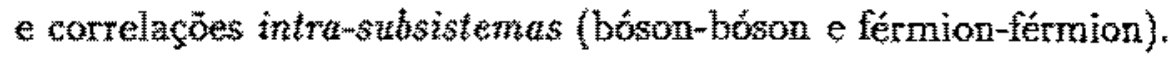

0 próximo passo consiste em usar o fato de que a decomposityo (3.24) pode ser implementada em termos de um operador de projeção dependente do tempo $P(t)=$ P(t)p(t) (c) Ret (3))

$$
P_{*}=F_{0} T r_{\mathrm{B}} \mathcal{P}_{\mathrm{B}}+\mathcal{F}_{0}^{\mathrm{a}} T r_{\mathrm{B}} \mathcal{P}_{\mathrm{F}}=-\mathcal{F}_{0}^{\mathrm{F}} \mathcal{F}_{0}^{\mathrm{B}} T r_{\mathrm{F}} T r_{\mathrm{B}}
$$

onde $P_{\text {n }}$ é operador de projeção dos bósons [33]

$$
\begin{aligned}
\mathcal{P}_{\mathrm{B}^{*}} & =\left[\left(1-\frac{\beta^{\dagger} \beta-\nu}{1+\nu}\right) \operatorname{Tr}_{\mathrm{B}}+\frac{\beta^{\dagger} \beta-\nu}{\nu(1+\nu)} \operatorname{Tr}_{\mathrm{B}}\left(\beta^{\dagger} \beta \cdot\right)+\frac{\beta}{\nu} \operatorname{Tr}_{\mathrm{B}}\left(\beta^{\dagger}\right)+\frac{1}{+}\right. \\
& \left.+\frac{\beta^{\dagger}}{1+\nu} \operatorname{Tr}_{\mathrm{B}}(\beta \cdot)+\frac{\beta \beta}{2 \nu^{2}} \operatorname{Tr}_{\mathrm{B}}\left(\beta^{\dagger} \beta^{\dagger}\right)+\frac{\beta^{\dagger} \beta^{\dagger}}{2(1+\nu)^{2}} \operatorname{Tr} r_{\mathrm{B}}(\beta \beta \cdot)\right] \mathcal{F}_{0}^{\mathrm{a}}
\end{aligned}
$$

$\mathcal{P}_{\mathrm{F}}$ é o projetor dos férmions [26]

$$
\mathcal{P}_{\mathrm{F}}=\left[\left(1-\sum_{\lambda=-1,1} \frac{\alpha_{\lambda}^{\dagger} \alpha_{\lambda}-p_{\lambda}}{1-p_{\lambda}}\right) T_{\mathrm{F}}+\sum_{\lambda, k=-1,1}\left(\frac{\left.\alpha_{\lambda}^{t} \alpha_{\lambda}-p_{\lambda} b_{\lambda \lambda}\right)}{p_{\lambda, \lambda}\left(1-p_{\lambda}\right)}\right) T_{F}\left(\alpha_{\lambda}^{t} \alpha_{\lambda}\right)\right] F_{0}^{F}
$$

e ponto indica objeto no qual o operador atua. Com ajuda de $p(t)$ a densidade gaussiana fatorizada pode ser obtida como

$$
F(t)=p(t) F
$$

enquanto a sua derivada temporal é dada pela expressà̃o

$$
\dot{F}_{0}(t)=\left[F_{0}(t), H\right]+P(t)[H, F]
$$

que é a representação de Heisenberg da exuaçào $\dot{P} F=0$ que foi usada pata definir $\mathcal{P}(t)$ na representaçăo de Schrödinger $[3,26]$.

A partir das eqs. (3.24) e (3.31) podemos obter uma equaço diferencial para $\mathcal{F}^{\prime}(t)$ entermos de $\mathcal{F}_{0}(t)$ 


$$
\left[i \frac{d}{d t}+\mathcal{P}(t) \mathcal{C}\right] \mathcal{F}^{\prime}(t)=\mathscr{Q L} \mathcal{F}_{0}(t)
$$

onde introduzimos o projetor complementar $\mathcal{Q}(t) \mathcal{F} \equiv[\mathcal{I}-\mathcal{P}(t)] \mathcal{F}=\mathcal{F}^{\prime}(t)$ e a notaçăó $\mathcal{L} \cdot$ para o Liouvilliano $[H, \cdot]$. Esta equação tem a solução formal

$$
\mathcal{F}^{\prime}(t)=\mathcal{G}(t, 0) \mathcal{F}^{\prime}(0)-i \int_{0}^{t} d t^{\prime} \mathcal{G}\left(t, t^{\prime}\right) \mathcal{Q}\left(t^{\prime}\right) \mathcal{L} \mathcal{F}_{0}\left(t^{\prime}\right)
$$

onde o primeiro termo corresponde às condiçóes iniciais (correlaçōes iniciais), e $\mathcal{Q}\left(t, t^{t}\right)$ é a funçà̃o de Green ordenada no tempo

$$
\mathcal{G}\left(t, t^{t}\right)=T \exp \left[i \int_{t^{t}}^{t} d r P(T) \mathcal{C}\right]
$$

Assim $\mathcal{F}^{\prime}(i)$, e consequentemente $\mathcal{F}$, podem ser formalmente expressas en termos de $F_{0}\left(t^{\prime}\right)$ para $t^{\prime}<t$ e das correlaçòes iniciais $\mathcal{F}^{\prime}(0)$. Isto finalmente nos permite expressar as equaçöes dinâmicas $(3.15),(3.19),(3,20),(3.22)$ e (3.22) como traços sobre funcionais de $\mathcal{F}_{0}\left(t^{\prime}\right)$ e das correlaçóes inciais $\mathcal{F}^{\prime}(0)$. Consequentemente, as equações dimârnicas que descrevem a evolução temporal de nosso sistema săo formalmente fecliadas em nossas variáveis de interesse.

\subsection{Aproximação para a Dinâmica de Colisões}

Como pode ser visto da equaçăo (3,33), a contribuiçăo de correlaçỏes às equaçôs dinâmicas envolvem além das correlaçoes iniciais, traços sobre funcionais de $F_{0}\left(t^{\prime}\right)$ com efeitos de memória dadas por expressões do tipo

$$
\begin{aligned}
& T_{T_{\mathrm{B}}}\left\{\mathcal{O}_{\mathrm{B}}, H\right] \int_{0}^{t} \mathcal{G}\left(t, t^{\prime}\right) Q\left(t^{\prime}\right) \mathcal{L} \mathcal{F}_{0}\left(t^{\prime}\right) d t^{t} \\
& T_{T_{\mathrm{BF}}}\left[\mathcal{O}_{\mathrm{F}}, H\right] \int_{0}^{t} \mathcal{G}\left(t^{\prime}, t^{\prime}\right) Q\left(t^{\prime}\right) \mathcal{L} \mathcal{F}_{0}\left(t^{\prime}\right) d t^{s}
\end{aligned}
$$

onde $\mathcal{O}_{\mathrm{B}}$ e $\mathcal{O}_{F}$ são densidades bosônicas e fermiônicas, respectivamente. A densidaté $\mathscr{L L F} \mathcal{F}_{\mathfrak{p}}=Q\left[H . \mathcal{F}_{0}\right]$ dada por 


$$
\begin{aligned}
& Q\left[H, \mathcal{F}_{0}\right]=\lambda\left\{\left(u^{*} v x-u z^{*} y\right)\left(\alpha_{1}^{\dagger} \alpha_{1} \frac{\beta}{\nu}-\alpha_{-1}^{1} \alpha_{-1} \frac{\beta}{\nu}\right)\right. \\
& +\left(u^{*} v y^{*}-u v^{*} x^{*}\right)\left(\alpha_{1}^{*} \alpha_{1} \frac{\beta^{\dagger}}{1+\nu}-\alpha_{-1}^{\dagger} \alpha_{-1} \frac{\beta^{t}}{1+\nu}\right) \\
& -\left(u^{*} v x-u v^{*} y\right)\left(p_{1}-p_{-1}\right) \frac{\beta}{v}-\left(u^{*} v y^{*}-u v^{*} x^{*}\right)\left(p_{1}-p_{-1}\right) \frac{\beta^{4}}{1+\nu} \\
& -\left(u^{* 2} z_{z}+v^{\pi 2} y\right)\left(\frac{p_{1}\left(1-p_{-1}\right)-\left(p_{-1}-p_{1}\right) \nu}{p_{-1}\left(1-p_{1}\right) t}\right) \alpha_{1}^{\dagger} \alpha_{-1} p \\
& -\left(u^{2} \hat{y}^{*}+v^{* 2} x^{*}\right)\left(\frac{p_{1}\left(1-p_{-1}\right)+\left(p_{-1}-p_{1}\right)(1+\nu)}{p_{-1}\left(1-p_{1}\right)(1+\nu)}\right) \alpha_{1}^{*} \alpha_{-1} \beta^{*} \\
& +\left(u^{2} y+v^{2} x\right)\left(\frac{p_{-1}\left(1-p_{1}\right)-\left(p_{1}-p_{-1}\right)}{p_{1}\left(1-p_{-1}\right) \nu}\right) \alpha_{-1}^{ \pm} \alpha_{1} \beta \\
& \left.+\left(u^{2} x^{*}+v^{2} y^{*}\right)\left(\frac{p_{-1}\left(1-p_{1}\right)+\left(p_{1}-p_{-1}\right)(1+v)}{p_{1}\left(1-p_{-1}\right)(1+\nu)}\right) a_{-1}^{t} \alpha_{1} \beta^{t}\right\} F_{0}
\end{aligned}
$$

representa as correlaçôes dinamicas (filtradas pelo projetor $Q \equiv I-P$ ) e como podemos ver, nào possui contribuiçôs de um corpo. Essas correlaçổs dinâmicas sâo entäo propagadas do instante $t$, no qual sâo produzidas, até o tempo $t$ por melo de $\mathcal{G}\left(t, t^{\prime}\right)$. Os traços

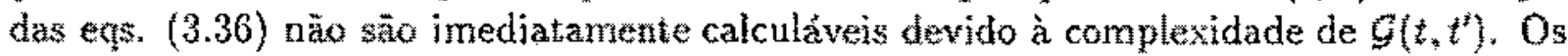
autores da ref. [26] mostraram uma maneira de aproximar o propagador $\mathcal{G}\left(t, t^{t}\right)$ na representaçăo de Schrodinger. Nesse trabaho eles expandem $G\left(t^{\prime \prime}\right)$ em séries de potencias de un propagador mais simples definido por

$$
\mathcal{G}_{0}\left(t, t^{*}\right)=T \exp \left\{i \int_{t^{2}}^{t} d+\mathcal{L}_{0}^{\mathrm{s}}(\tau)\right\}
$$

sendo a energia conservada em todas as ordens.

Seguindo Lin [33], implementamos na representação de Heisenberg uma versảo modificada da aproximaçăo de mais baixa ordem da rat, [20], que consiste em substítuir a hamiltoniana $H$ nas expressòes acima por uma hamiltoniana efetiva de campo medio $H_{0}$ dada por 


$$
\begin{aligned}
H_{0} & =p^{\dagger}(t) H+\beta^{\dagger} T r_{\mathrm{BF}}[\beta, H] \mathcal{F}^{t}(t)-\beta T r_{\mathrm{BF}}\left[\beta^{\dagger}, H\right] \mathcal{F}^{\prime}(t) \\
& +\frac{\beta^{\dagger} \beta^{\dagger}}{2(1+2 \nu)} \operatorname{Tr}_{\mathrm{GF}}[\beta \beta, H] \mathcal{F}^{t}(t)-\frac{\beta \beta}{2(1+2 \nu)} \operatorname{Tr}_{\mathrm{AF}}\left[\mathcal{F}^{\dagger} \beta^{\dagger}, H\right] \mathcal{F}^{\prime}(t) \\
& +\frac{\alpha_{1}^{\dagger} \alpha_{-1}}{\left(p-1-p_{1}\right)} \operatorname{Tr}_{\mathrm{BF}}\left[\alpha_{-1}^{\dagger} \alpha_{1}, H\right] \mathcal{F}^{\prime}(t)+\frac{\alpha_{-1}^{\dagger} \alpha_{1}}{\left(p_{1}-p_{-1}\right)} \operatorname{Tr}_{\mathrm{BF}}\left[\alpha_{1}^{\dagger} \alpha_{-1}, H\right] \mathcal{F}^{\prime}(t)
\end{aligned}
$$

O primeiro termo nesta expressão corresponde a aproximacăo en ordem mais baxa de acordo com a ref.[26]. Note que $P(t)$ não é uma projeção ortogonal, i.e. $P^{\dagger}(t) \neq P(t)$. $O s$ termos restantes incluidos agui representam contribuiçoes de correlaços ao campo médio eletivo. Calculando $P^{\dagger} H$ a partir dos projetores $(3.27),(3.28)$ e $(3.29)$, a hamiltoniana de campo médio $H_{0}$ pode ser reescrita como

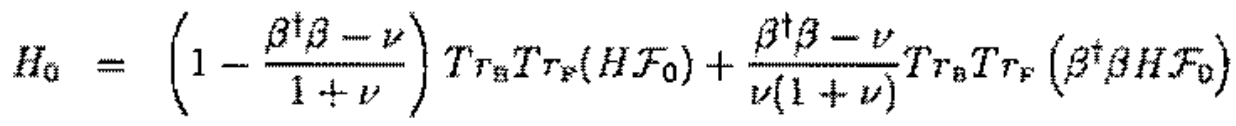

$$
\begin{aligned}
& -\not T_{B} T_{F}\left(\left[\beta^{\dagger}, H\right] \mathcal{F}\right)+\beta^{\dagger} T T_{B} T([\beta, H] \mathcal{F})+ \\
& -\frac{\beta \beta}{2(1+\nu)} \operatorname{Tr}{ }^{T} \gamma_{F}\left(\left[\beta^{\dagger} \beta^{\dagger}, H\right] F\right)+\frac{\beta^{\dagger} \beta^{\dagger}}{2(1+23)} \operatorname{Tr}_{B} T_{r_{F}}([\beta \beta, H] F)
\end{aligned}
$$

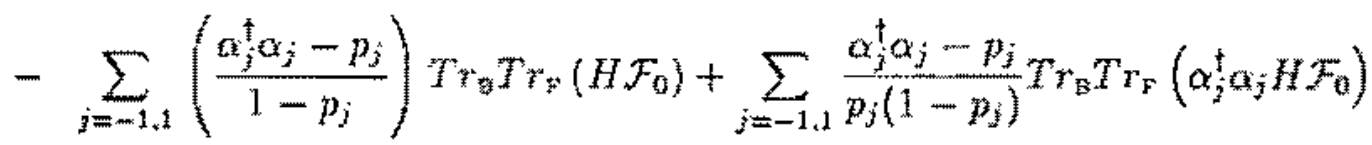

$$
\begin{aligned}
& +\sum_{j=-1, \mathfrak{1}(j \neq l)} \frac{\alpha_{j}^{\dagger} \alpha_{1}}{\left(p_{1}-p_{j}\right)} \operatorname{Tr}_{\mathrm{B}} \operatorname{Tr}_{\mathrm{F}}\left(\left(\alpha_{1}^{\dagger} \alpha_{j}, H\right] \mathcal{F}\right)-\operatorname{Tr}_{\mathrm{B}} \operatorname{Tr}_{\mathrm{F}}\left(H \mathcal{F}_{0}\right)
\end{aligned}
$$

Consistentemente com esta aproximaçäo, a funçäo de (reen $(3.34)$ em ordem mats baka na representaçăo de Heisenberg é dada pelo operador identidade (Veja Apêndice $B$ ]

$$
\mathcal{G}\left(t, t^{n}\right)=I
$$

de modo çue a densidade de correlaçäo nesta aproximaçầ fi dada por 


$$
F^{\prime}(t)=\mathcal{G}^{(0)}(t, 0) \mathcal{F}^{4}(0)-i \int_{0}^{t} d t^{t} \mathcal{Q}\left(t^{3}\right) \mathcal{L F}\left(t^{\prime}\right)
$$

Por simplicidade, nos restringiremos agora a estados inicialmente não correlacionados, i.e., $\mathcal{F}^{\prime}(0)=0$. As equaçöes dinâmicas das variáveis gaussianas com termos de correlação são então dadas por

$$
\begin{aligned}
& i \frac{d}{d t}\left[T_{\mathrm{BS}}\left(\mathcal{O}_{\mathrm{F}}(t) \mathcal{F}\right)\right]=\operatorname{Tr}_{\mathrm{BF}}\left[\mathcal{O}_{\mathrm{F}}(t), H\right] \mathcal{F}_{0}(t)-i \operatorname{Tr}_{\mathrm{gF}}\left[\mathcal{O}_{F}(t), H\right] \int_{0}^{i} d t^{\prime} Q\left(t^{\prime}\right) \mathcal{L} \mathcal{F}_{0}\left(t^{\prime}\right) \\
& \text { aprox. de campo mêdio termo de correlaţōes } \\
& i \frac{d}{d t}\left[\operatorname{Tr}_{\mathrm{BP}}\left(\mathcal{O}_{\mathrm{B}}(t) \mathcal{F}\right)\right]=\operatorname{Tr}_{\mathrm{BF}}\left[\mathcal{O}_{\mathrm{B}}(t), H\right] \mathcal{F}_{0}(t)-i \operatorname{Tr}_{\mathrm{BF}}\left[\mathcal{O}_{\mathrm{B}}(t), H\right] \int_{0}^{t} d t^{\prime} \mathcal{Q}\left(t^{\prime}\right) \mathcal{L} \mathcal{F}_{0}\left(t^{\prime}\right) \\
& \text { aprox. de campo médio termo de correlaçöes }
\end{aligned}
$$

onde $\mathcal{O}_{\mathrm{F}}$ e $\mathcal{O}_{\mathrm{B}}$ correspondem às variáveis gaussianas fermiônicas e bosônicas, respectivamente.

Os termos envolvendo $\mathcal{F}_{0}(t)$ podem ser calculados a partir da harniltoniana $H$ na base de quasin partículas e do operador de projeção dados por (3.27), (3.28) e (3.29). Contudo, os traços nos últínos termos das eqs. (3.41) não podem ser tomados diretamente, pois os operadores de quasi-particulas na integral e no primeiro comutador estão em tempos diferentes. Para resolver este problema consideremos a evolução temporal dos operadores $\beta(t), \alpha_{1}(t)=\alpha_{-1}(t)$, com o objetivo de verificar como estes operadores relacionam-se em tempos diferentes. A evolução temporal do operador $\beta(t)$ é dada pela equaçăo de Heisenberg,

$$
i \dot{\beta}=[\beta, H]+\frac{\partial \beta}{\partial t}
$$

onde o último termo leva em conta a dependència explícita de $\beta(t)$ com as amplitudes de deslocamento es efeitos de emparelhamento,

$$
\begin{aligned}
& i \dot{\beta}=[\beta, H]+i \frac{\partial \beta}{\partial x^{*}} \dot{x}^{*}+i \frac{\partial \beta}{\partial y^{*}} \dot{y}^{*}+i \frac{\partial \beta}{\partial B} \dot{B}+i \frac{\partial \beta}{\partial B^{*}} \dot{B}^{*}
\end{aligned}
$$

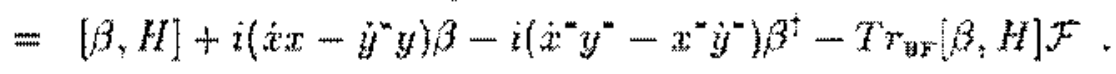


Analogamente, evolução temporal dos operadores fermiônicos $\alpha_{1}(t)$ e $\alpha_{-}$(l $(t)$ e dada por

$$
\begin{aligned}
\dot{i} \dot{\alpha}_{1} & =\left[\alpha_{1} H\right]+i \frac{\partial \alpha_{1}}{\partial u^{*}} \dot{u}^{*}+i \frac{\partial \alpha_{1}}{\partial v^{*}} \dot{v}^{*} \\
& =\left[\alpha_{1} H\right]+i\left(\dot{u}^{*} u+\dot{v}^{*} v\right) \alpha_{1}+i\left(\dot{u}^{*} v^{*}-u^{*} \dot{v}^{*}\right) \alpha_{-1}
\end{aligned}
$$

e

$$
\begin{aligned}
i \dot{\alpha}_{-1} & =\left[\alpha_{-1}, H\right]+i \frac{\partial \alpha_{-1}}{\partial u} \dot{v}+i \frac{\partial \alpha_{-1}}{\partial u} \dot{u} \\
& =\left[\alpha_{-1}, H\right]+i\left(\dot{u} u^{*}+\dot{v} v^{*}\right) \alpha_{-1}-i(\dot{u} v-\dot{u} \hat{v}) \alpha_{1} .
\end{aligned}
$$

Como loi dito anteriomente, vamos substituir a hamil toniana $H$ nas expressones acima por uma hamiltoniana efetiva de campo médio $H_{0}$ dada pela eci. (3.39),

$$
\begin{aligned}
& i \dot{\beta}=\left[\beta, H_{0}\right]+i\left(\dot{x}^{*},-\dot{y}^{*} y\right) \beta-i\left(\hat{x}^{*} y^{*}-x^{*} \dot{y}^{*}\right) \beta^{\dagger}-\mathrm{T}_{\mathrm{aF}}[\beta, H] F \\
& i \dot{\alpha}_{1}=\left[\alpha_{1}, H_{0}\right]+i\left(u^{*} u+\dot{w}^{*} v\right) \alpha_{1}+i\left(\dot{u}^{*} u^{*}-u^{*} \xi_{l}^{*}\right) \alpha_{-1} \\
& i \dot{\alpha}_{-1}=\left[\alpha_{i}, H_{0}\right]+i\left(u^{*} u+\dot{v}^{*} v\right) \alpha_{1}-i(\dot{u} v-u \dot{v}) \alpha_{-1} .
\end{aligned}
$$

Calculando os comutadores e reagrupando os termos, obtemos

$$
\begin{aligned}
& i \frac{d \beta}{d t}=i\left(\hat{a}^{2} x^{2}-\dot{y}^{m} y\right) \beta+\omega\left(|x|^{2}+|y|^{2}\right) \beta \equiv F_{\beta}(t) \beta \\
& i \frac{d \alpha_{1}}{d t}=\left[\frac{k}{2}\left(|n|^{2}-|v|^{2}\right)-\lambda\left(u^{\prime \prime} u B+u v^{*} B^{m}\right)\right] \alpha_{1}{ }^{*} \cdot\left(\dot{u}^{*} u+\dot{v}^{*} v\right) \alpha_{1} \equiv F_{\alpha_{1}}(t) \alpha_{1}
\end{aligned}
$$




$$
\begin{aligned}
i \frac{d \alpha_{-1}}{d t} & =-\left[\frac{\epsilon}{2}\left(|u|^{2}-|v|^{2}\right)-\lambda\left(u^{*} v B+u v^{*} B^{*}\right)\right] \alpha_{-1}+i\left(u^{*} u^{*}+\dot{v}^{*}\right) \alpha_{-1} \\
& =-\left[\frac{E}{2}\left(|u|^{2}-|v|^{2}\right)-\lambda\left(u^{*} v B+u v^{*} B^{*}\right)\right] \alpha_{-1}-i\left(u^{*} u+\dot{v}^{*} v\right) \alpha_{-1} \equiv F_{\alpha_{-1}}(i) \alpha_{-1}
\end{aligned}
$$

onde na thima passagem usamos o fato de que $u$ e $v$ satisfazem a relaçăo de unitariedade $|u|^{2}+|v|^{2}=1$

A partir de (3.49), (3.50) e (3.51) podemos verificar que os operadores $\beta(t)$ e $\alpha \lambda$ (t) en tempos diferentes estão relacionados por uma fase nessa aproximação

$$
i \frac{d \beta}{d t} \equiv F_{\beta}(t) \beta \Longrightarrow \beta(t)=e^{i \varphi_{\beta}\left\{t_{i} t^{7}\right\}} \beta\left(t^{\prime}\right)
$$

onde a lase $\varphi\left(t_{1} t^{\prime}\right)$ é dada por

$$
\varphi_{\beta}\left(t, t^{\prime}\right)=-\int_{t^{\prime}}^{t} d \tau F_{0}(\tau)
$$

e

$$
\begin{gathered}
i \frac{d \alpha_{1}}{d t} \equiv F_{\alpha_{1}}(t) \alpha_{1} \Longrightarrow \alpha_{1}(t)=e^{\left(\xi_{\mathrm{a}}\left(t_{t} t^{z}\right)\right.} \alpha_{1}\left(t^{\prime}\right) \\
\frac{d \alpha_{-1}}{d t} \equiv F_{\alpha_{-1}}(t) \alpha_{-1} \Longrightarrow \alpha_{-1}(t)=e^{-i \hat{\alpha}_{\alpha}\left(t, t^{\prime}\right)} \alpha_{-1}(t)
\end{gathered}
$$

onde a fase $\xi_{0}\left(t, t^{t}\right) \dot{e}$

$$
\xi_{o}\left(t, t^{t}\right)=-\int_{t^{*}}^{t} d r F^{\prime}(t)
$$

Sabendo que os operadores de quasi-particulas $\beta(t)$ e $\alpha$ (t) em tempos diferentes estäo relacionados por uma fase, podemos substituir as densidades fermiônicas botonicas em (3.41) e usando $(3.32-3.34)$ calculat o traço no instante $t^{\prime}$, Para uma dada densidade, $\mathcal{O}_{s}=a_{1}(t) \alpha_{1}(l)$ por exemplo, temos pata o termo de colisão 
$-i T T_{B H}\left[\mathcal{O}_{F}, H\right] \int_{0}^{t} d t^{t} Q\left(t^{\prime}\right) \mathcal{L} \mathcal{F}_{0}\left(t^{t}\right)$

$$
\begin{aligned}
& =-i T \tau_{\mathrm{BF}} \int_{0}^{t} d t^{t}\left[\alpha_{1}^{\dagger}(t) \alpha_{1}(t), H\right] \varrho\left(t^{t}\right) C F_{0}\left(t^{t}\right)
\end{aligned}
$$

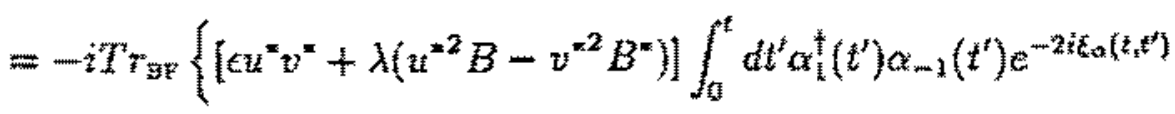

$$
\begin{aligned}
& -\left[\epsilon t v+\lambda\left(u^{2} B^{*}-v^{2} B\right)\right] \int_{0}^{t} d t^{t} \alpha_{-1}^{\dagger}\left(t^{\prime}\right) \alpha_{1}\left(t^{\prime}\right) e^{2 z_{\mathrm{a}}\left(t, t^{t}\right)} \\
& +\lambda\left[\left(u^{* 2} x+v^{* 2} y\right) \int_{0}^{t} d t^{\prime} \alpha_{1}^{\dagger}\left(t^{\prime}\right) \alpha_{-1}\left(t^{\prime}\right) \beta\left(t^{\prime}\right) e^{-2 i \varepsilon_{\alpha}\left(t, t^{\prime}\right)} e^{i \varphi_{\beta}\left(t, t^{\prime}\right)}\right. \\
& -\left(u^{* 2} y^{*}+v^{* 2} x^{*}\right) \int_{0}^{t} d t^{\prime} \alpha_{1}^{\dagger}\left(t^{\prime}\right) \alpha_{-1}\left(t^{\prime}\right) \beta^{\dagger}\left(t^{\prime}\right) e^{-2 i \xi_{\alpha}\left(t, t^{\prime}\right)} e^{-i w_{\alpha}\left(t, t^{\prime}\right)} \\
& +\left(u^{2} y+v^{2} x\right) \int_{0}^{t} d t^{\prime} \alpha_{-1}^{t}\left(t^{\prime}\right) \alpha_{1}\left(t^{\prime}\right) \beta\left(t^{\prime}\right) e^{2 i \varepsilon_{\alpha}\left[t_{1} t^{\prime}\right)} e^{i \psi_{\theta}\left(t_{1} t^{\prime}\right)}
\end{aligned}
$$

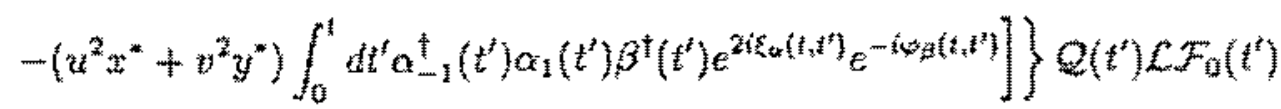

Substituindo a densidade $Q\left(t^{\prime}\right) \mathcal{L} \mathcal{F}_{0}\left(t^{\prime}\right)$ podemos calcular o traço obtendo para a integral de colisão deste exemplo a expressão

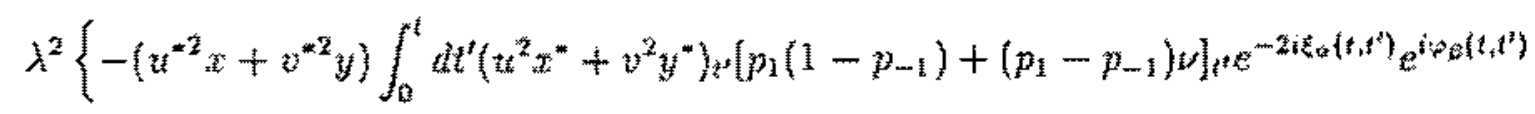

$$
\begin{aligned}
& +C . C \text {. } \\
& +\left(u^{-2} y^{*}+v^{-2} x^{*}\right) \int_{0}^{t} d t^{\prime}\left(z^{2} y+v^{2} x\right)_{t^{\prime}}\left[p_{-1}\left(1-p_{1}\right)+\left(p_{-1}-p_{1}\right) \nu\right]_{1} e^{-3 i \varepsilon_{0}\left(t, t^{\prime}\right)} e^{-i \varphi_{g}\left(t t^{\prime}\right)} \\
& + \text { C.C. }\}
\end{aligned}
$$

Finalmente. substituindo as demais densidades em (3.41) e calculando os traços, obtemos as equaçóes ọue descrevem a presente aproximaçào para a dinâmica de colisòes 


$$
\begin{aligned}
& \left.\dot{y}=f^{2}\left(w^{*} v y^{*}-w^{*} x^{*}\right) I_{1}+\left(z^{* 2} y^{*}+v^{m 2} x^{*}\right) I_{2}+\left(u^{2} x^{*}+v^{2} y^{*}\right) I_{3}\right\}+ \\
& +\mathrm{C} . \mathrm{O} \\
& \frac{d}{d t}\left(p_{1}-p_{m-1}\right)=2 \lambda^{2}\left\{-\left(u^{* 2} z+v^{* 2} y\right) I_{3}^{*}+\left(u^{x^{2}} y+v^{* 2} x^{*}\right) I_{2}\right\} \\
& +\mathrm{C} . \mathrm{C} \\
& i\left(p_{1}-p_{-1}\right)(\dot{u} v-u \hat{v})=\left(p_{1}-p_{-1}\right)\left\lfloor c u v+\lambda\left(u^{2} B^{*}-v^{2} B\right)\right]+ \\
& -2 i \lambda^{2}\left(u^{*} v x-u v^{*} y\right) I_{3}^{*}-2 i \lambda^{2}\left(u v^{*} x^{*}-u^{*} v y^{*}\right) I_{2}+ \\
& +i \lambda^{2}\left(u^{2} y+v^{2} x\right) I_{1}^{*}+i \lambda^{2}\left(u^{2} x^{*}+v^{2} y^{*}\right) I_{1} \\
& i(1+2 v)\left(\dot{x}^{*} y^{*}-x^{\prime \prime} \dot{y}^{\prime \prime}\right)=-(1+2 \nu) 2 \omega x^{*} y^{*}-2 i \lambda^{2}\left(u^{*} v y^{*}-u v^{*} x^{*}\right) l_{1}^{*}+ \\
& +2 i \lambda^{2}\left(u^{*} y^{*}+v^{-2} x^{*}\right) I_{3}^{*}+2 i \lambda^{2}\left(u^{2} x^{*}+v^{2} y^{*}\right) I_{2}^{*}
\end{aligned}
$$

onde

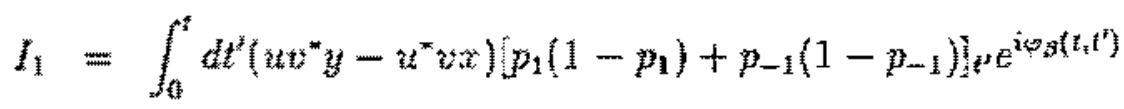

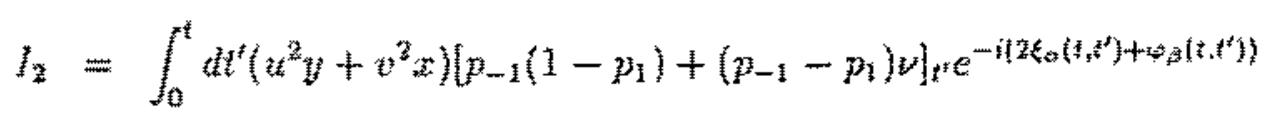

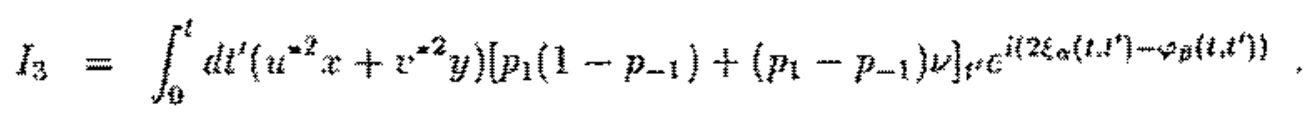

Observe que nào é possivel resolver o sistema de equaçoes tiferenciais (13.55 - 3.58) sem antes resolvermos as integrais $I_{1}, I_{2} \in I_{3}$. Usanto a equaça de fase (3.52), potenos escrever a integral $I_{1}$ como 


$$
I_{1}=e^{-I_{t}} I_{c}^{(1)}(t)
$$

onde

$$
\begin{aligned}
I_{\beta}(t) & =\int_{0}^{t} d t^{\prime} F_{\beta}\left(t^{\prime}\right) \\
I_{\varepsilon}^{(1)}(t) & =\int_{a}^{t} d t^{*}\left(u v^{*} y-u^{*} v x\right)\left[p_{1}\left(1-p_{1}\right)+p_{-1}\left(1-p_{-1}\right)\right]_{3} e^{i \varphi_{\beta}\left(t^{*}, 0\right)} \\
F_{\xi} & =i\left(\dot{x}^{*} x-\dot{y}^{*} u\right)+\omega\left(|x|^{2}+|y|^{2}\right)
\end{aligned}
$$

Para resolver a equaçäo $(3.62)$, escrevemos equacoes diferenciais para as integrais $I_{\beta}$ e $I_{e}^{(1)}$

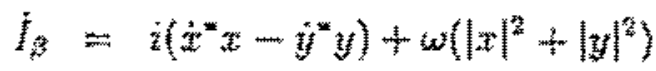

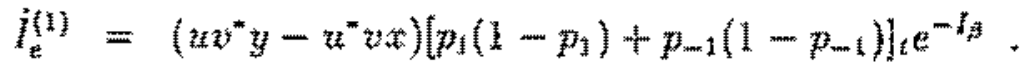

de modo que substituindo $I_{1}$ dada por $(3.62)$ nas equaçoes $(3.55-3.58)$, temos equações diferenciais para $I_{9}$ e $I_{e}^{(1)}$ dadas por $(3.66)$ e $(3.67)$ as quais podem ser integradas juntamente com as equaçòes $(3.55-3.58)$.

Usando a outra equaçäo de fase, obtemos a partir de $I_{2}$ e $I_{3}$

$$
\begin{aligned}
& f_{e}^{(2)}=\left(u^{2} y+v^{2} x\right)\left\lfloor p_{-1}\left(1-p_{1}\right)+\left(p_{-1} \cdots p_{1}\right) \nu\right]_{t} e^{-i\left(2 l_{a}+l_{\theta}\right)} \\
& f_{e}^{(3)}=\left(u^{-2} x+v^{-2} y\right)\left[p_{1}\left(1-p_{-1}\right)+\left(p_{1}-p_{-1}\right) u\right]_{l} e^{i\left(2 l_{a}-l_{A}\right)} \\
& \dot{l}_{\alpha}=i\left(\dot{u}^{*} u+\dot{v}^{*} v\right)+\frac{c}{2}\left(\left|u_{t}\right|^{2}-\left|v_{i}\right|^{2}\right)-\lambda\left(u_{*}^{*} v_{\mathrm{r}} B_{i}+u_{i} v_{i}^{*} B_{i}^{*}\right) .
\end{aligned}
$$

Usamos para os coeficientes da transformaçăo de Bogoliubov (3.10) a seguinte parametrização consistente com as condiçoes de unitariedade $(3.13)$ 


$$
\begin{aligned}
& u=\cos \theta \\
& y=e^{-i \tilde{a}} \sin \theta .
\end{aligned}
$$

Para os cofficientes da transformação de Bogoliubov dos bósons (3.5) a pararnetrização

$$
\begin{aligned}
& x=\cosh \kappa+i \frac{\eta}{2} \\
& y=\sinh x-i \frac{n}{2}
\end{aligned}
$$

consistente com as condiçôes de ortogonalidade e completera (3.8).

Assim podemos integrar as equaçoss $(3.66-3.70)$, juntamente com as equaçoes $(3.55$ - 3.58). Estas equaçōes descrevem a evolução temporal das variaveis gaussianas na aproximação com termos de colisäo.

\subsection{Resultados e Discussão}

Mostraremos agona os resultados obtidos para duas observáveis melevantes associadas ao subsistema fermionico (sistema de dois niveis) do Modelo de Jaynes-Cummings: a inversão atômica, $\left(\sigma_{3}(t)\right)$, e a inversấo atômica "intrínseca", denotada por $\left(\sigma_{p a t}(t)\right\rangle$, onde $\sigma_{p+1}$ a a projeçäo do operador $\vec{a}$ ao longo do eixo de polarizaçăo do spin, i.e,

$$
\sigma_{\mathrm{pol}}=\frac{\vec{a} \cdot|\vec{\sigma}|}{|(\vec{\sigma})|}
$$

e

$$
\sigma_{3}, \sigma_{ \pm}=\frac{\sigma_{1} \pm i \sigma_{2}}{2}
$$

são matrizes de Pauli. A inversão atômica intrínseca nos ká o grau de polarização intrinsecta do spin como funçào do tempo e depende da dinâmica de correlaçòes entre os subsistemas. En temos dos parämetros de Bogoliubov, os valores médios destas observáveis sảo dadas por 


$$
\begin{gathered}
\left\langle\sigma_{3}\right\rangle=\left(p_{1}-p_{-1}\right) \cos 2 \theta \\
\left\langle\sigma_{\mathrm{pol}}\right\rangle=\left(p_{1}-p_{-1}\right) .
\end{gathered}
$$

Um exemplo dos resultados obtidos nas aproximaçóes de campo médio e colisional para a evolução destes objetos é mostrada nas figuras abaixo. Elas foram obtidas integrandose numericamente as equą̧ôes $(3.55-3.58)$ e comparando-se com as soluçòes exatas do modelo.

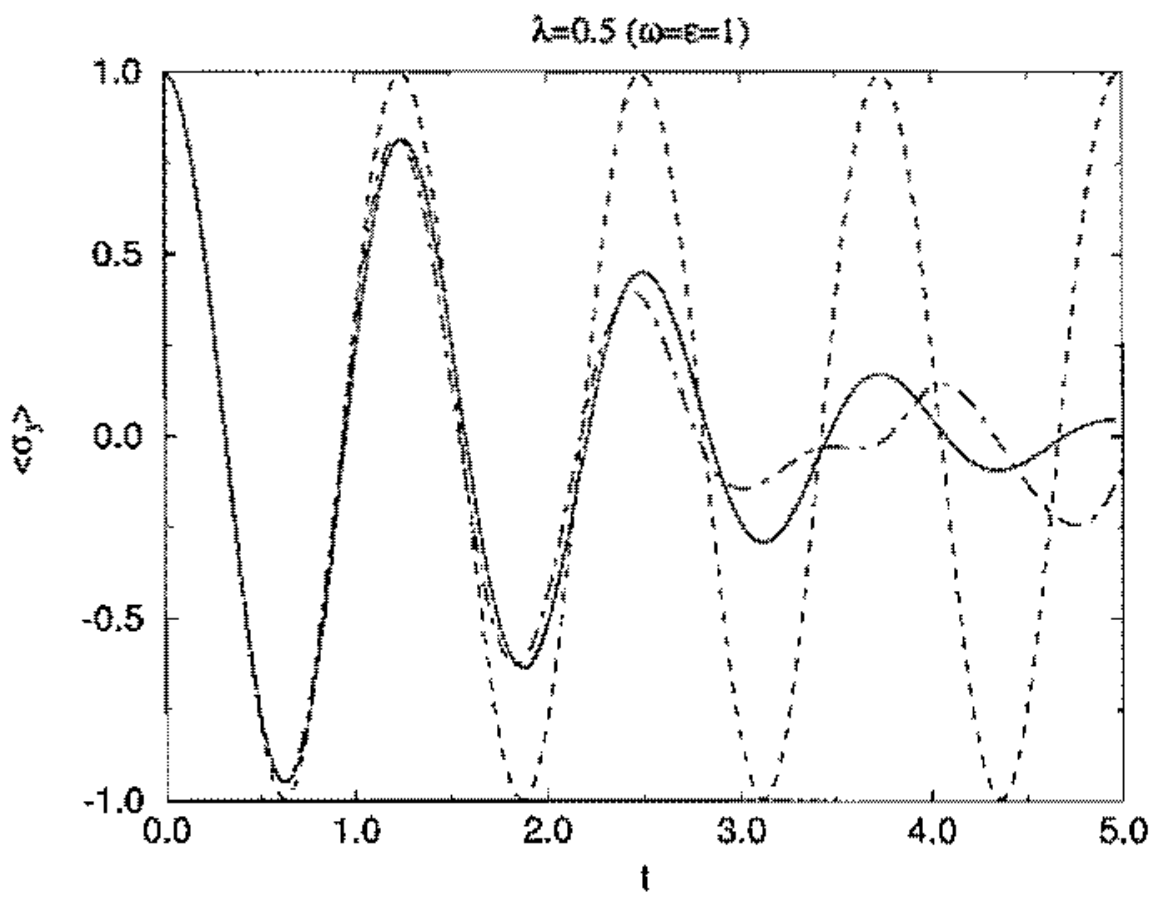

Figura 3.1: Evolução temporal da inversão atômica com $\lambda=0.5$. Condiçồes iniciais: $y=0, \hat{n}(0)=0, \eta(0)=0,|\mathcal{B}(0)|=5$ (estado coerente); $p_{1}=1, p_{-1}=0, \theta(0)=0$ e $\delta(0)=0.05$. Linha cheia: solução exatał Linha tracejada-pontilhada: aproximação colisional; Linha tracejada: aproximaçăo de campo médio 
A Figura 3.1 mostra a evoluçăo teroporal da inversẫo atồnica $\left\langle\sigma_{3}\right\rangle$ quando o átomo é inicialmente preparado no estado excitado $\left(p_{1}(0)=1, p_{-1}(0)=0, \partial(0)=0\right.$ e $\left.\delta(0)=0.05\right)$ e o campo esta inicialmente no estado coerente construido como o vácuo dos operadores de aniquilação deslocados $(\kappa(0)=0, \eta(0)=0$ and $\nu(0)=0)$. O número médio de fótons $B^{2}(0)$ é 25 neste exemplo. Nós usamos unidades tais que $\epsilon=\omega=1$ e $\lambda=0.5$. A evolução da polarizaçăo intrínseca para as mesmas condiçōes iniciais é mostrada na Figura 3.2.

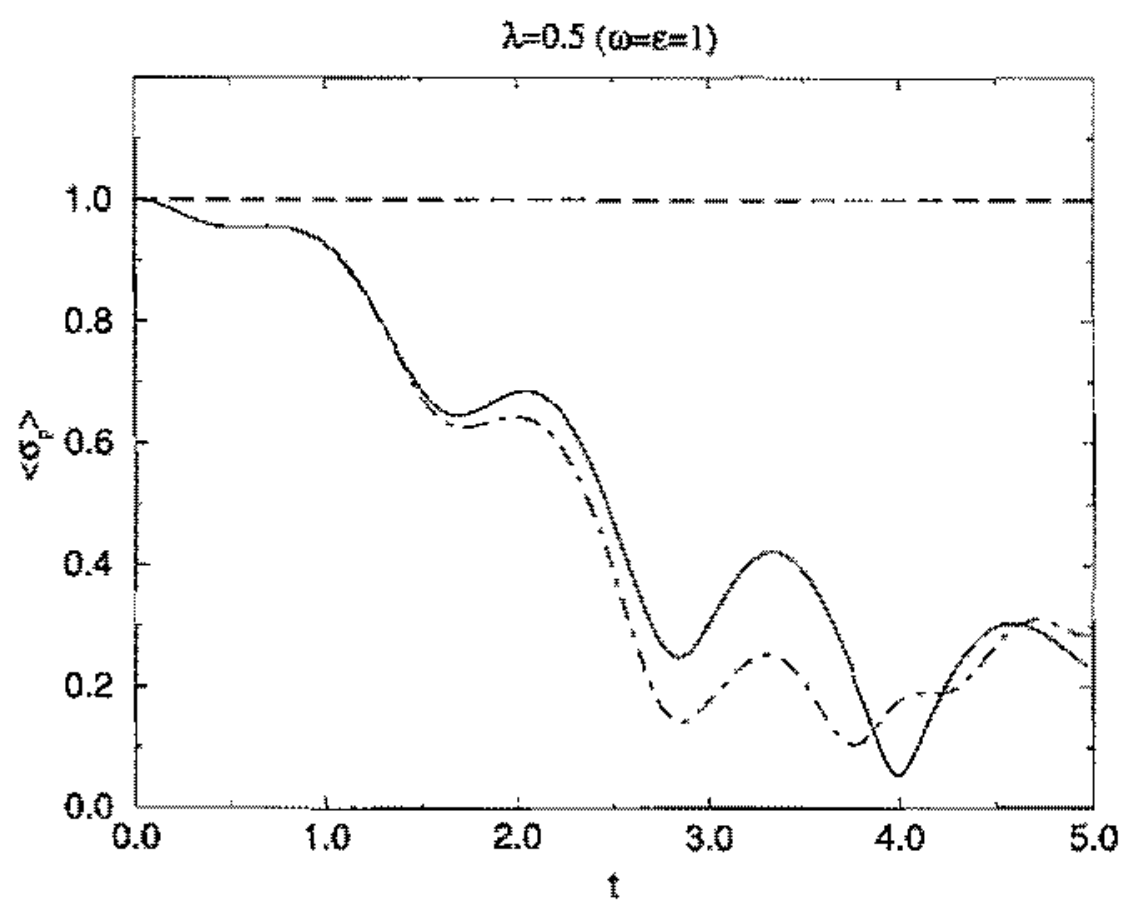

Figura 3.2: Evolução temporal da polarização intrinseca. As condiçôs iniciais são as mesmas solução exata; Linha tracejada-pontilhada: aproximação colisional Linha tracejada: aproximação de campo médio 
A aproximação de campo médio corresponde a ignorar as integrais $I_{1}, I_{2}$ and $I_{3}$, as quais são zero em todos os instantes nesta aproximação. Desta forma, as probabilidades de ocupação $v_{2} p_{1}$ e $p_{-1}$ tormam-se independentes do tempo, de molo que não existem correlaçóes entre os subsisternas. Como consequência, näo há despolarização e a inversảo atômica oscila com amplitude constante. Na aproximaçăo colisional, uma modulacão da amplitude das oscilações de Rabi é obtida a partir da despolarização resultante da inclusão dos termos de colisão, [eqs. (3.55) $(3.61)$ ], que descrevem as correlaçöes entre os subsistemas.

Concluimos que a inclusão d’̧ integrais de colisão nas equaçôs dinâmicas sâo não somente fundamentais para gerar o comportamento qualitativo associado com as correlaçôs entre os subsiatemas que resultam no amortecimento das oscilaçôs de Rabi, como tambén descreve cuantitativamente bern este comportamento para tempos comparảveis ao tempo de despolarizaçäo. 


\section{Capítulo 4}

\section{Equações Cinéticas na Teoria Quântica de Campos}

Neste capitulo adaptamos a técnica de projeção dependente do tempo descrita anteriorm mente ao estudo de sistemas relativisticos. Apresentamos assim, um tratamento năo perturbatío do problema de condição incial na Teoria Quântica de Campos para um sistema de férmions interagindo com bósons. A partir destas equaçōes discutimos as aproximaçôes conservativas năo pertubativas que tornam as equaçôes cinéticas obtidas traballhảveis.

\subsection{Caracterização das Variáveis de Interesse}

A idéia básica do nosso tratamento consiste no estudo da dinâmica efetiva do conjunto de variáveis de um corpo (densidades normal e de enparelhamento de um corpo) de um sistema de férmions interagindo com bósons escalares via uma interaçào do tipo Yukava. A lagrangiana que descreve tal sistema, contecido como plasma escalar, é dada por

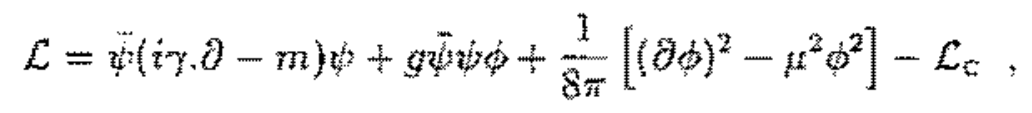

onde $\mathcal{L}_{c}$ compreende os contratermos necessấrios para renormalizar a teoria

$$
4 \pi c_{C}=\frac{A}{1 !} \phi+\frac{b_{1}^{2}}{2 !} \phi^{2}+\frac{C}{3 !^{3}}+\frac{D}{4 !} \phi^{4}-\frac{Z}{2}(0 \phi)^{2}
$$


Na eq. (4.1), $\phi(x)$ é un campo escalar com condiçós periódicas de contorno numa caixa de volume $V$, i.e.,

$$
\phi(x, t)=\sum_{0} \frac{1}{\left(2 V_{p}\right)^{1 / 2}}\left[b_{p}(t) e^{i p \cdot x}+b_{p}^{\dagger}(t) e^{-i p-x}\right]
$$

onde $b_{p}^{\dagger}(t)$ e $b_{p}(t)$ são operadores de criação e aniquilação de bósons, que satisfazem relaçoes de comutação a tempos iguais

$$
\begin{aligned}
& {\left[b_{p}(t), b_{p^{f}}^{\dagger}\left(t^{\prime}\right)\right]_{k=f^{*}}=\ddot{b}_{\mathrm{p}_{t} p^{*}}}
\end{aligned}
$$

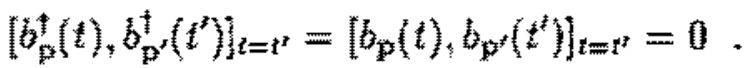

Na $e q .(4.3) \times$ denota a coordenada espacial e usamos a notação

$$
\left(p_{0}\right)^{2}=(\mathrm{p})^{2}+\Omega^{2} \text { e } p^{2}=p_{0} t-\mathrm{p} \cdot \mathrm{x}
$$

onde $\Omega$ é um parânetro de expansâo que será fixado mais tarde.

Por outro lado, na eq(4.1) $\eta_{(x)}(x)$ e $(x)$ sño campos de Dirac de spin-1/2 com condiços de contorno periódicas muma caxa de volmme $V_{\text {: }}$ definidos em termos do parânetro de expansāo

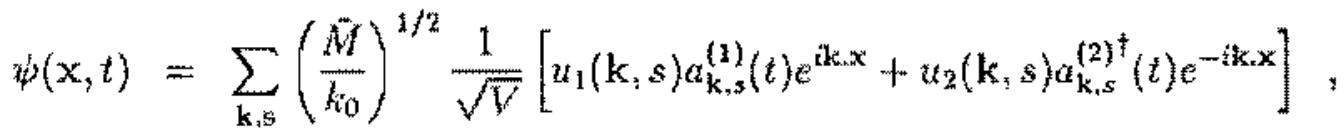

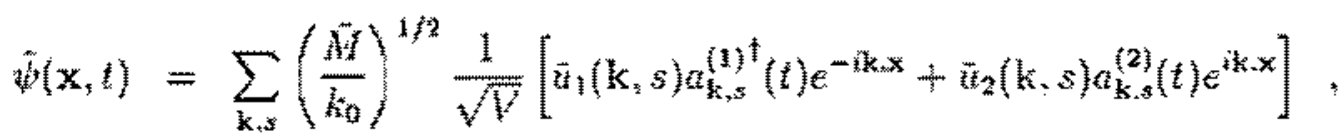

onde $a_{k, s}^{(n)^{t}}(t)$ e $a_{\mathrm{k}, s}^{(1)}(t)\left[a_{\mathrm{k}, s}^{(2)^{t}}(t)\right.$ e $\left.a_{\mathrm{k}, s}^{(2)}(t)\right]$ são operadores de criação e aniquilaçào de férmions associados com as soluçóes de energia positiva [negativa] $u_{1}\left(k_{*} s\right)\left[u_{2}\left(k_{1} s\right)\right]$ da equaçào de Dirac. Os operadores de criação e aniquilaçào de férmions satisfazem as relaços de anticomutaçăo a tempos fguais 


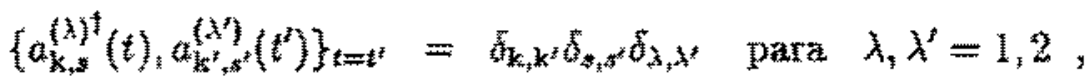

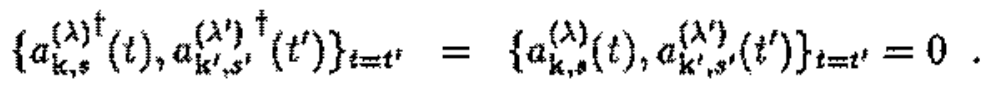

Analogamente ao caso do campo escalar, usamos na eq. (4.5) a notação

$$
\left(k_{0}\right)^{2}=(\mathrm{k})^{2}+\bar{M}^{2} \text { e } k x=k+k x
$$

O próximo passo é caracterizar as observáveis cuja evolução temporal desejanos estudar. O estado do sistema em geral dado em termos de um operador densidade de mutos corpos $\mathcal{F}$ na representação de Heisenberg. $\mathcal{F}$ um operador independente do tempo, hermitiano e com traço unitário. Particularizamos nosso estudo ao caso de um sistema espacialmente uniforme. Tal sistema exibe invariância translacional (homogêneo) e rotacional (isotrópico).

Uma maneira de introduzir nẩo uniformidades em nosso sistema seria quebrar a invam riancia translacional do mesmo, transformando-o num sistema nào homogêneo. Isto pode ser realizado via uma tranformação do tipo Bogoliubov nào homogênea para os operam dores de campo [35]. Outra mancira de introduzir năo uniformidades em nosso sisterna de muitos corpos seria atraves dos spinores de Dirac $u_{1}\left(k_{1} s\right)$ e $u_{2}\left(k_{3} s\right)$. Dependendo da representação que escolhemos para as matrizes $\gamma^{*}$; podemos quebrar a invariância por reflexăo (isotropia) de nosso sistema; neste caso, as equaçoes cinéticas que descrevem a evoluçào temporal de nosso sistema tornam-se nâo invariantes sob a transformaça $\mathrm{k} \rightarrow-\mathrm{k}$, o que obviamente năo traduz um sistema uniforme(veja apêndice $\mathrm{A}$ da ref. [19]). A simplificaçào introduzida no tratamento de sistemas uniformes provem das invariancias (simetrias) destes. Isto, de fato, faz com que a densidade a um corpo $\left\langle x|p| x^{\prime}\right\rangle$ de sistemas uniformes seja função apenas da quantidade invariante por translação e rotaçăo $\left|x-x^{*}\right|$. Neste caso os orbitais naturais sä̀ sempre ondas plantas.

Voltemos agora a caracterizar as variveis de um corpo de nosso sistema. Uma das variáveis de interesse é o valor esperado do campo bosônico

$$
\langle\phi(\mathbf{x}, t)\rangle=\sum_{\mathbf{p}} \frac{1}{\left(2 \hat{V} p_{0}\right)^{1 / 2}}\left[B_{\mathbf{p}}(l) e^{i \mathbf{p} \cdot \mathbf{x}}+B_{\mathrm{p}}^{*}(t) e^{-i p \cdot x}\right]
$$

$\operatorname{com}$

$$
B_{\mathrm{p}}(t)=T_{\mathrm{rg}} b_{\mathrm{p}}(t) \mathcal{F}
$$


onde $T r_{\text {top }}$ denota um traço sobre as variăveis bosonicas e fermionicas. Traços parciais sobre as variáveis bosơnicas ou fermiônicus serão escritos como $T_{r_{3}}$ e $T T_{7}$, respectivamente. No caso de sistemas uniformes a dependencia espacial do valor esperado do operador de campo deve ser eliminada. A partir de (4.7) vemos que isto é realizado impondo que

$$
B_{\mathrm{p}}(t) \stackrel{\text { uniforme }}{\longrightarrow} B_{\mathrm{p}}(t) d_{\mathrm{p}, 0}
$$

Assín, no caso uniforme o valor esperado do campo bosônico á dado por

$$
\langle(t)\rangle=\frac{1}{\sqrt{2 V \Omega}}\left(B(t)+B^{*}(t)\right)
$$

com

$$
B(t)=T_{\mathrm{p}} b_{0}(t) \mathcal{F}
$$

Analogamente, o valor esperado do openador momento canonico é escrito como

$$
(I I())=\sqrt{\frac{\Omega}{2 V}}\left(B^{*}-B\right)
$$

Consideremos agora a matriz densidade estendida de um corpo para um sistemaniforme de muitos bósonts [3]]

$$
R^{*}=\left[\begin{array}{cc}
\Lambda_{\mathrm{p}} & \Xi_{\mathrm{p}} \\
\Xi_{-\mathrm{p}} & 1+\Lambda_{-\mathrm{p}}
\end{array}\right]=\left[\begin{array}{cc}
\left\langle d_{\mathrm{p}}(t) d_{\mathrm{p}}(t)\right\rangle & \left\langle d_{\mathrm{p}}(t) d_{-\mathrm{p}}(t)\right\rangle \\
\left\langle d_{\mathrm{p}}^{\dagger}(t) d_{-\mathrm{p}}^{\dagger}(t)\right\rangle & \left\langle d_{\mathrm{p}}(t) d_{\mathrm{p}}(t)\right\rangle
\end{array}\right]
$$

onde a matria densidade nomal de um bóson $\Lambda_{p}$ é hermitiana e a matriz densidade de emparelhamento de um bóson $\equiv_{\mathrm{p}}$ é simétrica definimos os operadores teslocados

$$
d_{\mathrm{p}}(t)=b_{\mathrm{p}}(t)-B_{\mathrm{p}} \delta_{\mathrm{p}, \mathrm{p}}(t)
$$

os quais tem valor esperado nulo por construçăo. Observemos que no caso de sistemas näo uniformes, as densidades nomal e de emparelhamento de un bóson seriam 


$$
\begin{aligned}
& \Lambda_{\mathrm{p}, \mathrm{p}^{\prime}}=\operatorname{Tr}_{B F}\left[d_{\mathrm{p}}^{\dagger}(t) d_{\mathrm{p}^{\prime}}(t) \mathcal{F}\right] \\
& \Xi_{\mathrm{p}, \mathrm{p}^{\prime}}=\operatorname{Tr}_{B F}\left[d_{\mathrm{p}}(t) d_{\mathrm{p}^{\prime}}(t) \mathcal{F}\right] .
\end{aligned}
$$

enquanto no caso de sistemas uniformes as densidades $\Lambda_{p}$ e $\Xi_{p}$ dependem apenas do módulo do vetor momento, ou seja, $\mathrm{p}=|\mathrm{p}|$.

Caracterizemos agora as densidades fermiônicas de um corpo de sistema uniformes. No caso dos férmions a matriz densidade estendida de um corpo é

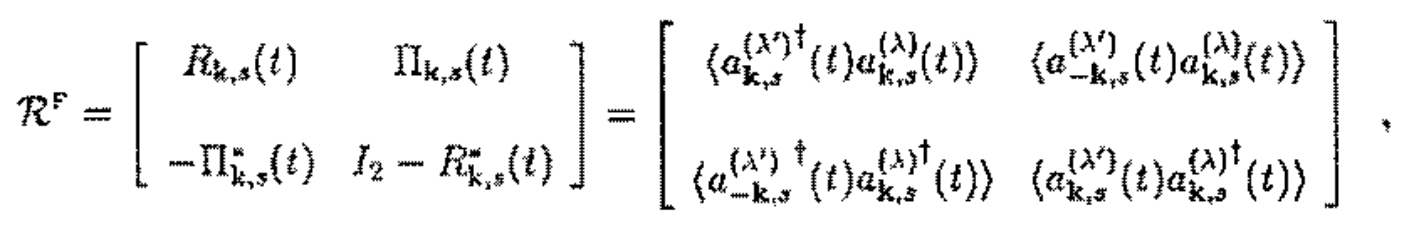

onde a matriz hermitiana $R_{k, a}$ ea matriz antissimétrica $\mathrm{I}_{k, s}$ são as densidades normal e de emparelhamento de um férmion, respectivamente. Novamente, para sistemas uniformes, estas densidade dependem apenas do módulo do momento, $\mathrm{k}=|\mathrm{k}|$.

Estes objetos dadós em $(4.9),(4.11),(4.12)$ e (4.14) para sistemas uniformes de muitos corpos contêm todas as informaçōes sobre as observáveis gaussianas e são o ponto de partida para nosso tratamento cinético.

Como foi dito no capitulo anterior, as matrizes densidades de un corpo $\mathcal{R}^{\mathrm{B}}$ e $\mathcal{R}^{\mathrm{q}}$ quando escritas na base de partículas são não diagonais, poís contém densidades de emparelhamento. Portanto, o primeiro passo consiste em diagonalizar $\mathcal{R}^{\mathbb{B}}$ e $\mathcal{R}^{F}$ através de uma transformaçăo de Bogoljubov. No caso dos bósons definimos operadores de quasibósons como

$$
\begin{aligned}
& \beta_{\mathrm{p}}(t)=x_{\mathrm{p}}^{*}(t)\left[b_{\mathrm{p}}(t)-B_{\mathrm{p}}(t) \delta_{\mathrm{p}, 0}\right]+y_{\mathrm{p}}^{*}(t)\left[b_{-\mathrm{p}}^{\dagger}(t)-B_{-\mathrm{p}}^{*}(t) \delta_{-\mathrm{p}, 0}\right] \\
& \beta_{\mathrm{p}}^{\dagger}(t)=x_{\mathrm{p}}(t)\left[b_{\mathrm{p}}^{\dagger}(t)-B^{m}(t) \delta_{\mathrm{p}, 0}\right]+y_{\mathrm{p}}(t)\left[b_{-\mathrm{p}}(t)-B_{-\mathrm{p}}(t) \delta_{-\mathrm{p}, 0}\right],
\end{aligned}
$$

onde $\beta_{\mathrm{p}}(t)$ e $\beta_{\mathrm{p}}^{\dagger}(t)$ satisfazem as mesma relaçoes de comutaçăo que $b_{\mathrm{p}}(t), b_{\mathrm{p}}^{\dagger}(t)$ em tempos iguais. 
Escrevendo na forma matricial temos

$$
\left[\begin{array}{c}
\beta_{p}(t) \\
\beta_{-\mathrm{p}}^{t}(t)
\end{array}\right]=\mathcal{X}_{\mathrm{p}}^{*}\left[\begin{array}{l}
d_{\mathrm{p}}(t) \\
d_{-\mathrm{p}}^{*}(t)
\end{array}\right]
$$

onde $X_{p}$ é dado por

$$
X_{\mathrm{p}}=\left[\begin{array}{ll}
x_{\mathrm{p}}(t) & y_{\mathrm{p}}^{*}(t) \\
z_{\mathrm{p}}(t) & x_{\mathrm{p}}^{*}(t)
\end{array}\right]
$$

Os coeficientes $x_{p}$ e $y_{p}$ da transformaçäo de Boyoliubov são determinados através do problema de autovalores que diagonaliza $\mathcal{R}^{\mathrm{B}}$

$$
G \mathcal{R}^{\mathrm{g}} \mathcal{X}_{\mathrm{p}}=\mathcal{X}_{\mathrm{p}} G N_{\mathrm{p}}
$$

onde

$$
G=\left[\begin{array}{cc}
1 & 0 \\
0 & -1
\end{array}\right], N_{\mathrm{p}}=\left[\begin{array}{cc}
\nu_{\mathrm{p}} & 0 \\
0 & 1+u_{\mathrm{p}}
\end{array}\right]
$$

e a matriz $\mathcal{X}_{\mathrm{p}}$ é dada na eq. (4.17). Os autovalores $v_{p}$ podem ser interpretados como o número de ocupação dos bósons deslocados para os orbitais naturais emparelhados descritos por $\mathcal{X}_{\mathrm{p}}$, ou em outras palavras, sào as ocupaçós de quasi-bósons deslocados. Enfim, urna vez que a transformagăo de Bogoliubov échonica pode-se verificar que $X_{p}$ satislaz as relaçòes de completeza e ortogonalidade

$$
X_{p}^{\dagger} G X_{p}=X_{p} G X_{p}^{\dagger}=G \Rightarrow\left|x_{p}\right|^{2}-\left|y_{p}\right|^{2}=1
$$


Analogamente, para os operadores fermiontoos definimos a transformaçăo

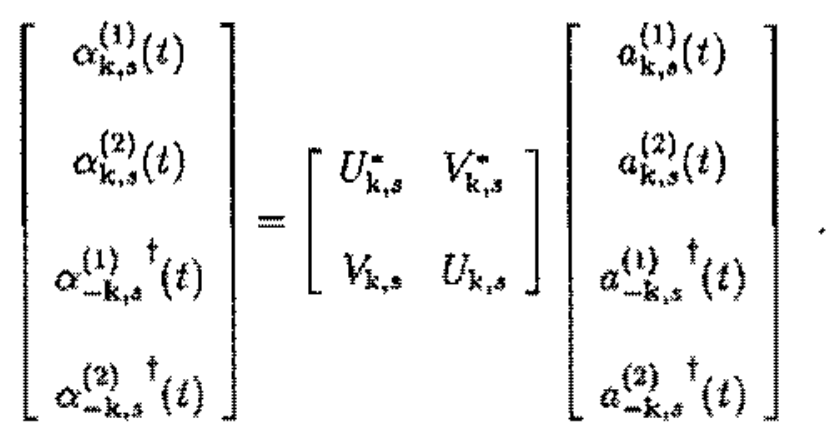

Como no caso dos bósons, temos um problema de autovalores que diagonaliza $\mathcal{R}^{F}$

$$
\mathcal{X}_{\mathrm{k}, \mathrm{s}}^{\mathrm{t}} \mathcal{R}^{\mathrm{F}} \mathcal{X}_{\mathrm{k}, \mathrm{s}}=N_{\mathrm{k}, \mathrm{s}}^{\mathrm{t}}
$$

onde $\mathcal{X}_{k,}$ é a transformaçäo definida ern $(4,21)$, ou seja,

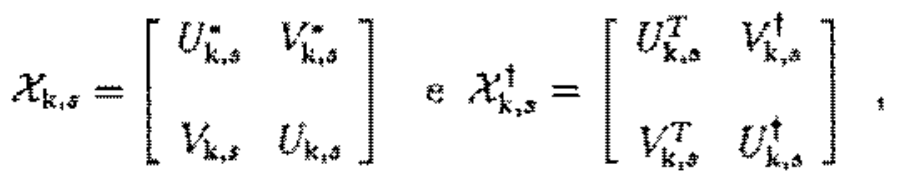

satisfazendo as equaçōes de unitariedade

$$
\mathcal{X}_{\mathrm{k}, s}^{\ddagger} \mathcal{X}_{\mathrm{k}, \mathrm{s}}=\mathcal{X}_{\mathrm{k}, a} \mathcal{X}_{\mathrm{k}, \mathrm{s}}^{\ddagger}=\mathrm{I}_{4}
$$

e onde as matrizes $U_{\mathrm{k}, \mathrm{s}}$ e $V_{\mathrm{k}, \mathrm{s}}$ são dadas por

$$
U_{\mathrm{k}, s}=\left[\begin{array}{cc}
U_{11} & 0 \\
0 & U_{2 z}
\end{array}\right] \text { e } V_{\mathrm{k}_{2},}=\left[\begin{array}{cc}
0 & V_{12} \\
V_{21} & 0
\end{array}\right] \text {. }
$$

Finalmente, a matriz $N_{k, s}$ é a matriz densidade estendja diagonalizada de um férmion na base de quastufếrmions 


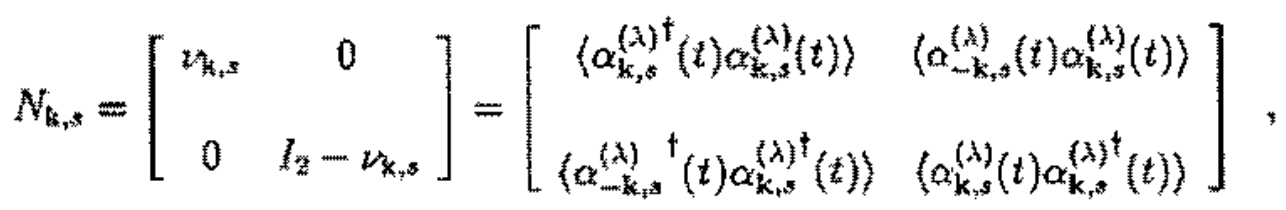

onde $\nu_{\mathrm{k}, 3}$ e matriz dentidade normal de um fémion na base de quasi-partículas. Nesta base $\nu_{k, s}$ é diagonal com autovalores $v_{k_{k}}^{(1)}$ e $v_{k, s}^{(2)}$ que correspondem aos números de ocupação dos respectivos estatos (orbitais naturais).

Devemos notaz que devido ao fato de estarmos tratando sistemas uniformes, as ocupaçoes e os coeficientes de Bogoliubov dependem somente do módulo do momento, ou seja,

$$
\begin{aligned}
& t_{\mathrm{p}}=\nu_{\mathrm{p}}, x_{\mathrm{p}}=t_{\mathrm{p}}, y_{\mathrm{p}}=y_{\mathrm{p}} \\
& \nu_{k, s}^{(\lambda)}=y_{k, s)}^{(\lambda)}, U_{i j}(k, s)=U_{i j}(k, s), V_{i j}\left(k_{2} s\right)=V_{i j}(k, s) .
\end{aligned}
$$

Antes de obter as equaçōes dinâmicas de nosso sistema co conveniente analisarmos um pouco melhor as transtormaçoes de Bogoliubox para os operadores de campo bostrico e fermiónico, definidas em (4.16) e (4.21). Primeiramente, observemos que através de (4.16) e (4.21) poderiamos ter introduzido inomogeneidades em nosso sistema (como já foi dito anteriomente), caso partíssemos com os operadors de campo $t_{p}(t), d_{\mathrm{p}}^{\dagger}(t), a_{\mathrm{k}, \mathrm{s}}^{t}(t)$ e $a_{\mathrm{k},{ }^{\dagger}}^{\dagger}$ e deixássemos que as matrizes de Bogoliubor $X_{\mathrm{p}}$ e $\mathcal{X}_{\mathrm{k}_{\mathrm{q}} \mathrm{s}}$ dependessem de todos os momentos $\mathrm{k}^{\prime}$ e $\mathrm{p}^{\prime}$. Deste modo, um operador de campo na base de quasimparticulas seria uma somatória en $\mathrm{k}^{\prime}$ (inclusive para $\mathrm{k}=\mathrm{k}^{\prime}$ ) da combinaçào linear de todos os outros operadores da base de particulas. Neste caso as densitades de um torpo do tipo

$$
\begin{aligned}
& A_{\mathrm{p}^{*} \mathrm{p}}=\mathrm{Tr}_{\mathrm{p}}\left[\beta_{\mathrm{p}^{*} \beta_{\mathrm{p}}}^{\dagger} \mathcal{F}\right]
\end{aligned}
$$

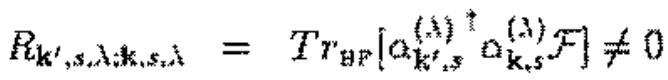

seriam não nulas para $\mathrm{p}^{\prime} \neq \mathrm{p}$ e $\mathbf{k}^{\prime} \not 1 \mathrm{k}$, pois a transformacão de Bogoliubov geral introduta correlaçoes entre os vários momentos, enquanto os campos $(x), f(x)$ e $\phi(x)$. escritos na 
base de quasi-partículas de Bogoliubov, seriam näo uniformes (näo seriam invariantes por translaçâ). Observe que no caso de uma transformafão de Bogoliubov uniforme do tipo (4.16) e (4.21) temos para as densidades de um corpo

$$
\begin{aligned}
& \Lambda_{\mathrm{p}^{\prime}, \mathrm{p}}=T_{T_{\mathrm{BF}}}\left[\beta_{\mathrm{p}^{\prime}}^{\dagger} \beta_{\mathrm{p}}{ }^{2}\right]=\nu_{\mathrm{p}} \delta_{\mathrm{p}^{\prime}, \mathrm{p}}
\end{aligned}
$$

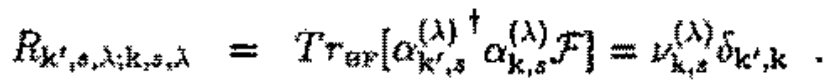

Desta obșervaçăo fica claro que o estudo de sistemas não uniformes extremamente mais complicado, do ponto de vista computacional, quando comparado ao estudo de sistemas uniformes. Logo, nossa abordagem restringir-se-á ao estudo de sistema uniformes.

Outra observaçào que devemos fazer com relação a transformação (4.21) refere-se ao fato de que estaremos contiderando estados de helicidade definida. Em ontras palavras, nầo estamos misturando o número quântico de spin. Na referência [9] (veja artigo de Celenza e Shakin) a quebra desta simetria neste modelo é discutida em detalhes.

Finalmente, observemos que a transformaçäo definida em $(4.21),(4.23)$ e $(4.25)$ é um caso particular da transformação uniforme geral de Bogolubov, pois conserva a simetria de carga de nosso modelo (4.1). Para verificar isto, escrevamos explicitamente a densidade sem carga $a_{k, s}^{(1)} a_{k, s}^{(1)}$ na base de quasiwférmions,

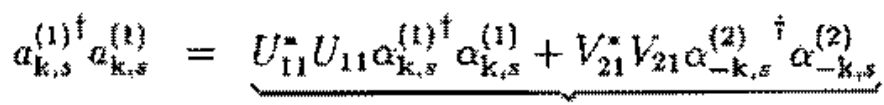

$$
\begin{aligned}
& \text { densidades normais sem carga } \\
& +\underbrace{U_{1 \mathrm{~g}} \nu_{12} \alpha_{-k, s}^{(2)} \alpha_{k, s}^{(1)}+U_{11}^{*} V_{12}^{*} \alpha_{k, s}^{(1)} \alpha_{-k, 3}^{(2)}}
\end{aligned}
$$

de onde observamos imediatamente que a simetria $U(1)_{\text {sass }}$ nào quebrada. Este lipo particular de transformaça de Bogoliubov também é conhecida como transformaçăo de Nambu (veja artigo de Nambu e Jona-Lasinio em (9)).

\subsection{Dinâmica das Variáveis de Interesse}

O estudo da dinâmica das variáveis de interesse se restringe ao estudo das observảveis $\langle\phi(t)\rangle,\langle\Pi(t))$ e dos coefictentes da transformaçă de Bogoliubov dados pelas matrizes $X_{\rho}$. $U_{k, s} \in V_{k, s}$. 
Começamos com o subsistema bosônico. A equação dinâmica para o valor esperado do campo escalar $\langle\hat{\varphi(t)})$ resulta diretamente da equaçăo de Heisenberg

$$
i\langle\dot{\phi}(t)\rangle=T_{r_{\mathrm{BF}}}[\phi(t), H] \mathcal{F},
$$

onde $H$ é a hamiltoniana do sistema e $(\phi(t))$ édado pela eq. (4.9). Escrevendo a equaçäo dinẩica em termos das amplitudes $B(t)$, pois a parte real de $B(t)$ é proporcional à $(\phi(t)\}$ e a parte imaginária de $B(t)$ é proporcional à $\langle(\Pi(t))$, temos

$$
i \dot{B}_{\mathrm{p}} \delta_{\mathrm{p}, 0}=x_{\mathrm{p}} \operatorname{Tr}_{\mathrm{aF}}\left[\beta_{\mathrm{p}} \hat{\delta}_{\mathrm{p}, 0}, H\right] \mathcal{F}-y_{\mathrm{p}}^{*} T r_{\mathrm{BF}}\left[\beta_{-\mathrm{p}}^{\dagger} \delta_{-\mathrm{p}, 0}, H\right] \mathcal{F} .
$$

Para estudar a dinâmica das demais variáveis bosônicas, é necessário obter a evolução temporal da matriz densidade estendida $\mathcal{R}^{\sharp}$. Primeiramente reescrevemos a equação de autovalores (4.28), usando $(4.20)$, como

$$
\mathcal{X}_{\mathrm{p}}^{\dagger} R^{\mathrm{B}} \mathcal{X}_{\mathrm{p}}=N_{p}
$$

Derivando corn relação ao tempo temos

$$
\begin{aligned}
\mathcal{X}_{\mathrm{p}}^{+} \dot{\mathcal{R}}^{\mathrm{B}} \mathcal{X}_{\mathrm{p}} & =\dot{N}_{\mathrm{p}}-\dot{\mathcal{X}}_{\mathrm{p}}^{\dagger} R^{\mathrm{a}} \mathcal{X}_{\mathrm{p}}-\mathcal{X}_{\mathrm{p}}^{\dagger} R^{\mathrm{a}} \dot{X}_{\mathrm{p}} \\
& =\dot{N}_{\mathrm{p}}-\dot{\mathcal{X}}_{\mathrm{p}}^{\dagger} G \mathcal{X}_{\mathrm{p}} G N_{\mathrm{p}}-N_{\mathrm{p}} G \mathcal{X}_{\mathrm{p}}^{\dagger} G \dot{X}_{\mathrm{p}}
\end{aligned}
$$

Como estamos trabalhando na representação de Heisenberg, a matriz densidade completa $\mathcal{F} \dot{e}$ independente do tempo, enquanto o operador matriz densidade estendida de

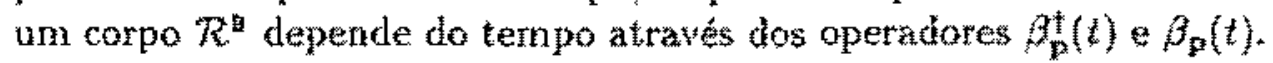

$O$ lado esquerdo da eq.(4.30) pode ser calculado derivando-se com relaçăo ao tempo as matrizes densidades normal e de emparelhamento e utilizando-se a equaç̧̃o de Heisenberg.

$$
i X_{\mathrm{p}}^{\dagger} \dot{\mathcal{R}}^{\mathrm{B}} \chi_{\mathrm{p}}^{\dagger}=\left(\begin{array}{cc}
\operatorname{Tr}_{\mathrm{BF}}\left[\beta_{\mathrm{p}}^{\dagger} \beta_{\mathrm{p}}, H\right] \mathcal{F} & \operatorname{Tr}_{\mathrm{BF}}\left[\beta_{\mathrm{p}} \beta_{-\mathrm{p}}, H\right] \mathcal{F} \\
\operatorname{Tr}_{\mathrm{BF}}\left[\beta_{\mathrm{p}}^{\dagger} \beta_{\mathrm{p}}^{\dagger}, H\right] \mathcal{F} & \operatorname{Tr}_{\mathrm{BF}}\left[\beta_{\mathrm{p}} \beta_{\mathrm{p}}^{\dagger}, H\right] \mathcal{F}
\end{array}\right) .
$$

$O$ lado direito pode ser obtido substituindo-se (4.17) e (4.19) e usando as relaşóes de completeza e ortogonalidade (4.20). Igualando o resultado à eq.(4.31), obtemos as equaçóes que descrevem a evolução temporal das variáveis bosônicas 


$$
\begin{aligned}
& \dot{i}_{\mathrm{p}}=\operatorname{Tr}_{\mathrm{BF}}\left[\beta_{\mathrm{p}}^{\dagger} \beta_{\mathrm{p}}, \ldots\right] F \\
& \left(1+2 \nu_{p}\right)\left(\dot{x}_{p} y_{p}-x_{p} \dot{y}_{p}\right)=T r\left[\rho_{p}^{\dagger} \beta_{-p}^{\dagger} H\right] \mathcal{F}
\end{aligned}
$$

onde $x_{\mathrm{p}}$ e $y_{\mathrm{p}}$ são os coefictentes da transformação de Bogoliubov e $\nu_{\mathrm{p}}$ a a ocupação de quasi-bósons definida em (4.19),

$$
z_{\mathrm{p}}=\left\langle\beta_{\mathrm{p}}^{\dagger} \beta_{\mathrm{p}}\right\rangle
$$

Temos agora que obter cquaçōes análogas para as variaveis fermiônicas. Derivando con relaçäo to tempo a eq, (4.22) e usando as equaçöes de unitariedade $(4.24)$, obtemos

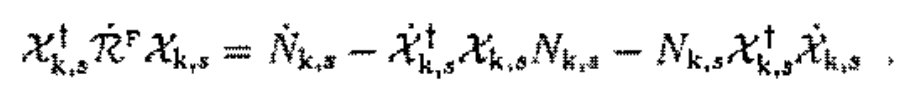

Substituindo $(4.23),(4.25)$ e (4.26) podemos calcular o lado direito da ef( $(4.33)$, obtendo a seguinte equaçào matricial

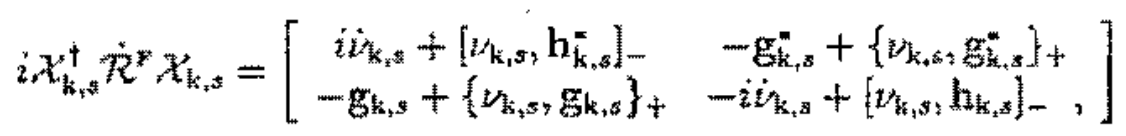

onde $\{\}+$, denota um anticomutador e [, , un comtatador e as matrizes $h_{k, s}$ e $g_{k, s}$ são dadas em termos das matrizes $U_{k, z} e V_{k, s}$

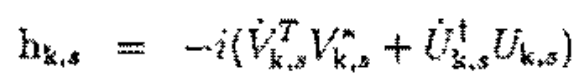

$$
\begin{aligned}
& g_{k, s}=-i\left(\hat{v} \hat{k}_{t}^{t} U_{k, s}^{*}+\dot{l}_{k, s}^{\dagger} V_{k, s}\right) .
\end{aligned}
$$

Como no caso dos bósons, a matriz densidade estendida de um férmion $R^{\mathrm{F}}$ depende do tempo através dos operadores $\alpha_{k, s}^{(\lambda)^{t}}(t)$ e $\alpha_{k, 5}^{t, k}(t)$. Para calcular o lado esquerto da eq. $(4.33)$, utilizamos a ecuaçäo de Heisenberg; além da invarianncia ciclica do traço. Obtemos assim a equaçōes gue descrevem a linâmica das vanùveis fermiônicas 


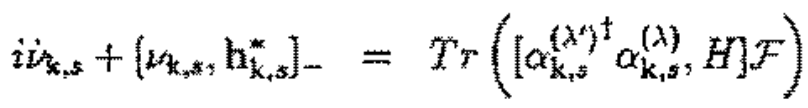

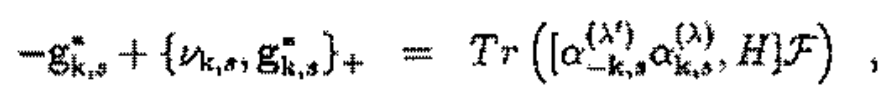

onde as matrizes $h_{k, s}$ e $g_{k, s}$ stäo dadas em $(4.35)$ e $z_{k, s}$ é a matriz de ocupaçäo de quasiférmions de Nambu definida em (4.26),

$$
n_{k, s}=\left\langle\alpha_{k_{1}, s}^{(\lambda)} \alpha_{k, s}^{(\lambda)}\right\rangle=\left[\begin{array}{cc}
v_{k, s}^{(i)} & 0 \\
0 & w_{k, s}^{(2)}
\end{array}\right] .
$$

Finalmente devernos observar que as variâvels de interesse, quando escritas na base de quasti-partículas, contém os coeficientes da transformaçào de Bogoliubov, os quais juntamente com as ocupaçôes $\nu_{\mathrm{p}}$ e $\nu_{\mathrm{k}, \mathrm{s}}^{(\lambda)}$ constituem as varít veas cuja evoluçâo desejamos estudar.

Como no caso do Nodelo de Jaynes-Cummings, as equs. (4.28), (4.32) e (4.36) não sẫo fechadas em nossas variáveis, pois dada a matria densidade completa de muitos corpos as equaçôs dependem da hamiltoniana do sistema que contern interacöes de dois corpos (termos de interação entre os subsistemas) de modo que as equaçós que descrevem a dinatmica de $R^{B}$ e $R^{F}$ também envolverào densidades de dois corpos.

\subsection{Técnica de Projeção Dependente do Tempo}

Corm o objetivo de tratar as equaçỏ de movimento obtidas na seção anteriot começamos dividindo a densidade completa F como no capitulo anterior

$$
\begin{aligned}
\mathcal{F} & =\mathcal{F}_{0}(t)+\mathcal{F}^{2}(t) \\
& =\mathcal{F}_{0} \mathcal{F}_{0}+\mathcal{F}^{\prime}(t)
\end{aligned}
$$

onde a forma fatoriada de $F_{0}(t)$ corresponde à tapla aproximaço de campo médio. As densidade dos subsistemas bosonico e fermionico $\mathcal{F}_{0}^{\mathrm{B}}$ e $\mathcal{F}_{0}^{F}$ sta densidade gautstanas escritas na loma de uma exponencial de uma expresão hermitiana bilinear nas partes

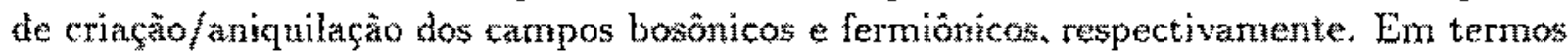
dos operadores transformados introduzidos em (4.15) e (4.21) são dadas por [02] 


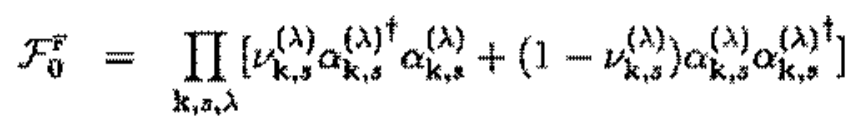

$$
\begin{aligned}
& \mathcal{F}_{0}^{\mathrm{a}}=\prod_{\mathrm{p}} \frac{1}{1+\nu_{\mathrm{p}}}\left(\frac{\nu_{\mathrm{p}}}{1+\nu_{\mathrm{p}}}\right)^{\beta_{p} a_{\mathrm{p}}}
\end{aligned}
$$

onde $T r_{\text {g }} \mathcal{F}_{0}^{g}(t)=T r_{F} \mathcal{F}_{0}(t)=1$. Como no capitulo unterior, os traços dos operadores $\alpha_{k_{s} s}^{(\lambda)}, \alpha_{k, s}^{(\lambda)}, \beta_{p}^{\dagger}$ e $\beta_{p}$ com a densidade $\mathcal{F}_{0}=\mathcal{F}_{0}^{g} \mathcal{F}_{0}^{7}$ são dados por

$$
\begin{aligned}
& T F_{\operatorname{man}}\left(\alpha_{c} F_{0}\right)=T T_{\mathrm{ar}}\left(\alpha_{\mathrm{a}}^{t} \mathcal{F}_{0}\right)=0 \\
& \operatorname{Tr}_{B y}\left(\alpha_{\alpha} \alpha_{b} F_{0}\right)=\operatorname{Tr}_{B F}\left(\alpha_{s}^{t} \alpha_{b}^{t} F_{0}\right)=0 \\
& \operatorname{Tr}_{\mathrm{eF}}\left(\alpha_{a}^{\dagger} \alpha_{b} \mathcal{F}_{0}\right)=v_{a} b_{a b} \\
& T_{T_{\mathrm{BF}}}\left(\alpha_{\alpha} \alpha_{b}^{*} \tau_{0}\right)=\left(1-v_{a}\right) \delta_{\mathrm{at}} \\
& \operatorname{Tr}_{\mathrm{B}}\left(B \mathcal{F}_{0}\right)=\operatorname{Tr}_{\mathrm{BF}}\left(\mathrm{OP}_{\mathrm{a}} F_{0}\right)=0
\end{aligned}
$$

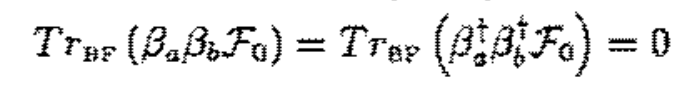

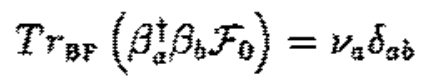

$$
\begin{aligned}
& \mathrm{Tr}_{\mathrm{BF}}\left(\beta_{\mathrm{B}} \beta_{b}^{t} F_{0}\right)=\left(1+\nu_{B}\right) \hat{b}_{a b} \\
& T v_{\mathrm{EF}}\left(\beta_{\mathrm{a}}^{\dagger} \rho_{b}^{t} \beta_{s} \beta_{d} \mathcal{F}_{0}\right)=\nu_{\mathrm{a}} \nu_{b}\left(\delta_{\mathrm{a}} \hat{\delta}_{b d}+\hat{\delta}_{\mathrm{at}} \delta_{\mathrm{b}}\right) \text {. }
\end{aligned}
$$

A densidade $F(t)$, que tem traço nulo, descreve a parte de correlaçóes da densidade completa Devido a forma $(4.37)$ usada para a densidade $\mathcal{F}_{0}(t), \mathcal{F}$ em geral contém correlacoes de dois tipos: correlaçôes intê-subsistemas (bóson-fórmion) e intra-\$ubsistemas (bóson-bóson e férmion-férmion).

O próxino passo consiste em usar o fato de que a decomposiçäo (4.37) pode ser implementada em termos de um operador de projeção dependente do tempo $P(t)=$ $P(1) P(t)$ [29]. Corn a ajuda de $\mathcal{P}(t)$ a densidale gaussiana fatorizada pode ser obtida como

$$
F_{0}(t)=P(t) \mathcal{F}
$$


enquanto a sua derivada em relação ao tempo é dada por

$$
i \dot{\mathcal{F}}_{0}(t)=\left[\mathcal{F}_{0}(t), H\right]+\mathcal{P}(t)[H, \mathcal{F}]
$$

que é a representação de Heisenberg da equação $\dot{\mathcal{P}} \mathcal{F}=0$ usada para definir $\mathcal{P}(t)$ na representação de Schrödinger $[3,26]$.

A partir das eqs. (4.37) e (4.41) podemos obter uma equação diferencial para $\mathcal{F}^{\prime}(t)$ em termos de $\mathcal{F}_{0}(t)$

$$
\left[i \frac{d}{d t}+\mathcal{P}(t) \mathcal{L}\right] \mathcal{F}^{\prime}(t)=\mathcal{Q} \mathcal{L} \mathcal{F}_{0}(t)
$$

onde introduzimos o projetor complementar $\mathcal{Q}(t) \mathcal{F} \equiv(\mathcal{I}-\mathcal{P}(t)) \mathcal{F}=\mathcal{F}^{\prime}(t)$ e a notação $\mathcal{L} *$ para o Liouvilliano $[H, *]$. Esta equação tem a solução formal

$$
\mathcal{F}^{\prime}(t)=\mathcal{G}(t, 0) \mathcal{F}^{\prime}(0)-i \int_{0}^{t} d t^{\prime} \mathcal{G}\left(t, t^{\prime}\right) \mathcal{Q}\left(t^{\prime}\right) \mathcal{L} \mathcal{F}_{0}\left(t^{\prime}\right)
$$

onde o primeiro termo leva em conta as correlações iniciais e $\mathcal{G}\left(t, t^{\prime}\right)$ é a função de Green ordenada temporalmente

$$
\mathcal{G}\left(t, t^{\prime}\right)=\mathrm{T} \exp \left[i \int_{t^{\prime}}^{t} d \tau \mathcal{P}(\tau) \mathcal{L}\right]
$$

Assim, a densidade $\mathcal{F}^{\prime}(t)$, e consequentemente $\mathcal{F}$, podem ser formalmente expressas em termos de $\mathcal{F}_{0}\left(t^{\prime}\right)$ para $t^{\prime}<t$ e das correlaçỏes iniciais $\mathcal{F}^{\prime}(0)$. Finalmente, isto nos permite expressar as equações dinâmicas (4.28). (4.32) e (4.36) em termos de traços sobre funcionais de $\mathcal{F}_{0}\left(t^{\prime}\right)$ e das correlaçòes iniciais $\mathcal{F}^{\prime}(0)$.

\subsection{Aproximação de Campo Médio}

O nosso objetivo é obter a dinâmica efetiva das variáveis de interesse para o sistema uniforme do plasma escalar. A aproximaçào de campo médio consiste em tomar $\mathcal{F}^{\prime}(t)=0$, ou seja,

$$
\mathcal{F} \longrightarrow \mathcal{F}_{0}(t)
$$


Logo, nesta aproximaçẫo consideramos que não há correlaçôes dinâmicas no sistema, exceto as correlaçôes de emparelhamento introduzidas através da transformaģăo de Bogoliubov.

A matriz densidade de muitos corpos $F_{0}$ mais geral que descreve completamente os aspectos de um corpo é dada por

$$
\mathcal{F}_{0}=\mathcal{F}_{0}^{F} \mathcal{F}_{0}^{B}
$$

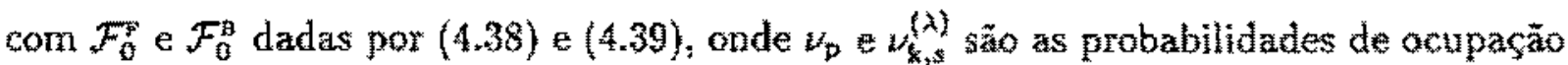
dos respectivos orbitais naturais, ou seja, as densidades de um corpo na base de quasiparticulas. Substituindo (4.45) nas eqs. $(4.28),(4.32)$ e $(4.36)$ obtemos as equaços que descrevem a dinamica efetiva das densidades de um corpo do plasma escalar na aproximaçäo de campo medio

$$
\begin{aligned}
& i \dot{B}_{\mathrm{p}} \delta_{\mathrm{p}, 0}=x_{\mathrm{p}} \operatorname{Tr}_{\mathrm{BF}}\left[\beta_{\mathrm{p}} \delta_{\mathrm{p}, 0}, H\right] \mathcal{F}_{0}-y_{\mathrm{p}}^{*} \operatorname{Tr}_{\mathrm{aF}}\left[\beta_{-\mathrm{p}}^{\dagger} \delta_{-\mathrm{p}, 0}, H\right] \mathcal{F}_{\mathrm{v}} \\
& \dot{\nu}_{\mathrm{p}}=\operatorname{Tr}_{\mathrm{BF}}\left[\beta_{\mathrm{p}}^{\dagger} \beta_{\mathrm{p}, H} H\right] \psi_{0} \\
& i\left(1+2 u_{p}\right)\left(\dot{x}_{p} \xi_{p}-x_{p} z_{p}\right)=\operatorname{Tr}\left[\beta_{p}^{\dagger} \beta_{-p}^{\dagger} H\right] \mathcal{F}_{0}
\end{aligned}
$$

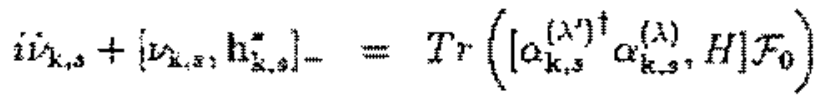

$$
\begin{aligned}
& -\mathrm{g}_{k, s}^{*}+\left\{\nu_{\mathrm{k}_{s}, s}, \mathrm{~g}_{\mathrm{k}, s}^{*}\right\}_{+}=\operatorname{Tr}\left(\left[\alpha_{-\mathrm{k}_{, s}}^{\left(\lambda^{\prime}\right)} \alpha_{\mathrm{k}, s}^{(\lambda)}, H\right] \mathcal{F}_{0}\right)
\end{aligned}
$$

onde as matrizes $h_{k, 5}$ e $g_{k_{1} s}$ são dadus em $(4.35)$ e $\mathcal{F}_{0}$ en $(4.3 \%$. 4.39$)$.

No próximo capitulo obteremos as equaçoss cineticas que descrevem a evoluça a tenporal do plasma escalar na aproximaçà de campo médio e a partir ta tas equaçoes estáticas renormalizaremos a teoria. 


\section{Capítulo 5}

\section{Dinâmica Efetiva para o Plasma Escalar Relativístico na Aproximação de Campo Médio}

Como no Modelo de Jaynes-Cummings, o qual corresponde ao plasma escalar em $(0+1)$ dimensōes, obteremos neste capitulo equações do tipo cinético que descreverão a evolução temporal do plasma escalar relativístico. A partir do estudo das soluçōes estáticas renormalizaremos a teoria. Embora nảo represente um sistema físico real, o plasma escalar é extremamente útil para a implementação de aproximaçōes necessárias no estudo de modelos mais realísticos.

\subsection{Dinâmica de Campo Médio para o Plasma Esca- lar}

A densidade de hamiltoniana para o plasma escalar é dada por

$$
\mathcal{H}=-\bar{\psi}(i \vec{\gamma} \cdot \vec{\partial}-m) \psi-g \bar{\psi} \phi \psi+\frac{1}{8 \pi}\left((4 \pi)^{2} \Pi^{2}+|\partial \dot{\phi}|^{2}+\mu^{2} \phi^{2}\right)
$$

A partir da densidade de hamiltoniana podemos obter a hamiltoniana do sistema bastando integrar (5.1) em todo espaço

$$
H=\int d \times \mathcal{H}
$$

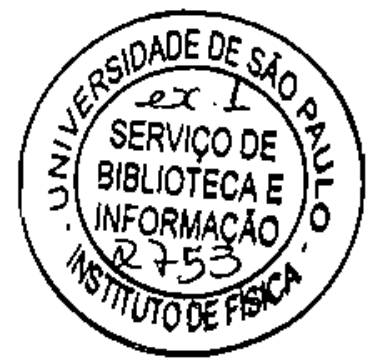


Para isto, consideramos o campo escalar e o campo de Dirac na representação de Heisenberg dados por (4.3) e (4.5). Como já foi dito anteriormente, as soluções de energia positiva e negativa $u_{1}(\mathbf{k}, s)$ e $u_{2}(\mathbf{k}, s)$ da equação de Dirac podem quebrar a invariância por reflexão de nosso sistema. No apêndice A da referência [19] foi feito um estudo que permite diferenciar as várias representaçōes das matrizes $\gamma^{\mu}$ de Dirac em $(1+1)$ dimensões invariantes por reflexão. Vamos considerar a representação de Pauli-Dirac para as matrizes $\gamma^{\mu}$ em $(3+1)$ dimensões, ou seja,

$$
\gamma^{0}=\left(\begin{array}{cc}
I & 0 \\
0 & -I
\end{array}\right), \gamma^{i}=\left(\begin{array}{cc}
0 & \sigma^{i} \\
-\sigma^{i} & 0
\end{array}\right) .
$$

Nesta representação as soluçōes da equação de Dirac são

$$
u_{1}(\mathbf{k}, s)=\left(\frac{k_{0}+\bar{M}}{2 \bar{M}}\right)^{1 / 2}\left(\begin{array}{c}
\chi_{s} \\
\frac{\sigma \cdot \mathrm{k}_{\chi_{s}}}{\left(k_{0}+\bar{M}\right)}
\end{array}\right) \quad u_{2}(\mathbf{k}, s)=\left(\frac{k_{0}-\bar{M}}{2 \bar{M}}\right)^{1 / 2}\left(\begin{array}{c}
\chi_{s} \\
\frac{\sigma \cdot \mathbf{k}_{\chi_{s}}}{\left(k_{0}-\bar{M}\right)}
\end{array}\right)
$$

as quais conservam a invariância por reflexão de nosso sistema e onde $\bar{M}$ é o parâmetro de expansão introduzido na equação (4.5). As soluções de Dirac (5.4) obedecem às relações

$$
\begin{aligned}
& \bar{u}_{1}\left(\mathbf{k}, s^{\prime}\right) u_{1}(\mathbf{k}, s)=-\bar{u}_{2}\left(\mathbf{k}, s^{\prime}\right) u_{2}(\mathbf{k}, s)=\delta_{s, s^{\prime}} \\
& \bar{u}_{1}\left(\mathbf{k}, s^{\prime}\right) u_{2}(\mathbf{k}, s)=\bar{u}_{2}\left(\mathbf{k}, s^{\prime}\right) u_{1}(\mathbf{k}, s)=0 \\
& u_{1}^{\dagger}\left(\mathbf{k}, s^{\prime}\right) u_{1}(\mathbf{k}, s)=u_{2}^{\dagger}\left(\mathbf{k}, s^{\prime}\right) u_{2}(\mathbf{k}, s)=\frac{k_{0}}{M} \delta_{s, s^{\prime}} \\
& u_{1}^{\dagger}\left(\mathbf{k}, s^{\prime}\right) u_{2}(-\mathbf{k}, s)=u_{2}^{\dagger}\left(\mathbf{k}, s^{\prime}\right) u_{1}(-\mathbf{k}, s)=0 .
\end{aligned}
$$

Substituindo (4.3), (4.5), (5.3) e (5.4) em (5.2) e utilizando-se as propriedades dos spinores (5.5), podemos fazer a integraçào espacial e obtemos assim a hamiltoniana do plasma escalar

$$
H=H_{\mathrm{D}}+H_{\mathrm{kG}}+H_{\mathrm{l}}
$$

onde 


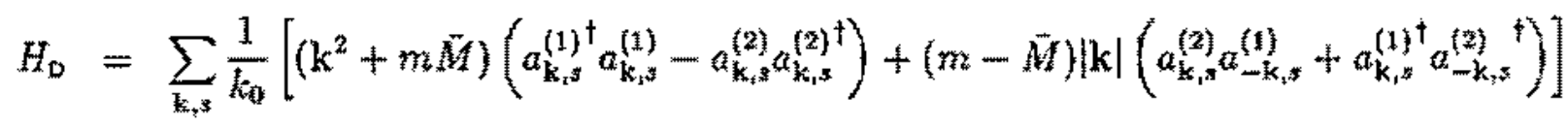

$$
\begin{aligned}
& H_{\mathrm{KG}}=\frac{1}{16 \pi} \sum_{\mathrm{p}} \frac{1}{\left(\mathrm{p}^{2}+\Omega^{2}\right)^{1 / 2}}\left\{\left((4 \pi)^{2}\left(\mathrm{p}^{2}+\Omega^{2}\right)+\left(\mathrm{p}^{2}+\mu^{2}\right)\right]\left(b_{\mathrm{p}} b_{\mathrm{p}}^{\dagger}+b_{\mathrm{p}}^{\dagger} b_{\mathrm{p}}\right)\right. \\
& \left.+\left\{\left(\mathrm{p}^{2}+\mu^{2}\right)-(4 \pi)^{2}\left(\mathrm{p}^{2}+\Omega^{2}\right)\right]\left(b_{\mathrm{p}} h_{-\mathrm{p}}+b_{\mathrm{p}}^{\dagger} b_{-\mathrm{p}}^{\dagger}\right)\right\} \\
& H_{1}=-g \sum_{s, s^{3}} \sum_{\mathbf{k}, \mathbf{p}} \frac{\bar{M}}{\left(k_{0}(\mathrm{k}) k_{0}(\mathrm{p}-\mathrm{k})\right)^{1 / 2}} \frac{1}{\left(2 V p_{0}\right)^{1 / 2}} \\
& \times\left\{\bar{u}\left(\mathrm{k}-\mathrm{p}, s^{t}\right) u(\mathrm{k}, s) a_{\mathrm{k}, \mathrm{p}, s^{\prime}}^{(1)}(t) a_{\mathrm{k}, s}^{(\mathrm{l})}(t)\left(\mathrm{b}_{-\mathrm{p}}(t)+b_{\mathrm{p}}^{\dagger}(t)\right)\right. \\
& +n\left(-\mathrm{k}+\mathrm{p}, s^{\prime}\right) v(-\mathrm{k}, s) a_{-\mathrm{k}+\mathrm{p}, \mathrm{s}^{t}}^{(2)}(t) a_{-k, s}^{(2)}(t)\left(b_{-\mathrm{p}}(t)+b_{\mathrm{p}}^{\dagger}(t)\right) \\
& +\bar{u}\left(\mathrm{k}-\mathrm{p}, s^{\prime}\right) u(-\mathrm{k}, s) a_{\mathrm{k}-\mathrm{p}, s^{\prime}}^{(1)}(t) a_{-\mathrm{k}, \mathrm{s}}^{(2)}(t)\left(b_{-\mathrm{p}}(t)+b_{\mathrm{p}}^{\dagger}(t)\right) \\
& \left.+\tilde{w}\left(-\mathrm{k}+\mathrm{p}, s^{t}\right) u(\mathrm{k}, s) a_{-\mathrm{k}+\mathrm{p}, s^{t}}^{(2)}(t) a_{\mathrm{k}, 5}^{(1)}(t)\left(b_{m} \mathrm{p}(t)+b_{\mathrm{p}}^{\dagger}(t)\right)\right\} \text {. }
\end{aligned}
$$

A partir da hamiltoniana $(5.6-5.9)$ e da matriz densidade $\mathcal{F}_{0}(t)$ dada por $(4.37-4.39)$, podemos obter a dinămica efetiva das variáveis de interesse, on seja, do valor esperado do campo bosốnico e das matrizes densidade estendidas de um corpo $\mathcal{R}_{\mathrm{I}} \mathrm{e} \mathcal{R}_{\mathrm{F}}$ na aproximaçăo de campo médio para o plasma escalar.

Conecemos reescrevendo as equaçōes $(4.47-4.49)$ que descrevem a dinảmica das variáveis bosônicas na aproximaçăo de campo médio

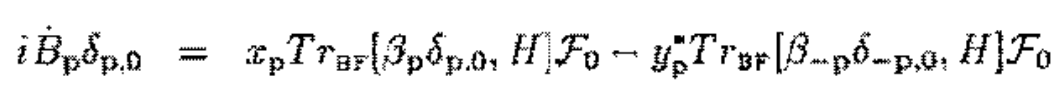

$$
\begin{aligned}
& \ddot{i \hat{\nu}_{\mathrm{p}}}=\operatorname{Tr}_{\mathrm{BF}}\left[\beta_{\mathrm{p}}^{\dagger} \beta_{\mathrm{p}} \cdot H\right] \mathcal{F}_{0} \\
& i\left(1+2 \nu_{\mathrm{p}}\right)\left(\dot{x}_{\mathrm{p}} z_{\mathrm{p}}-\alpha_{\mathrm{p}} \hat{y}_{\mathrm{p}}\right)=T_{\gamma_{\mathrm{BF}}}\left[\beta_{\mathrm{p}}^{\dagger} \beta_{-\mathrm{p}}^{\dagger}, H\right] F_{0}
\end{aligned}
$$


onde $\nu_{\mathrm{p}}$ é a ocupação de quasimbósons de Bogoliubov e $x_{\mathrm{p}}$ e $y_{\mathrm{p}}$ são coeficientes da transformaçầo de Bogoliubov.

Para as variáveis fermiônicas, a dinânica efetrva é dada pelas equaçòes $(4.50-4.51)$

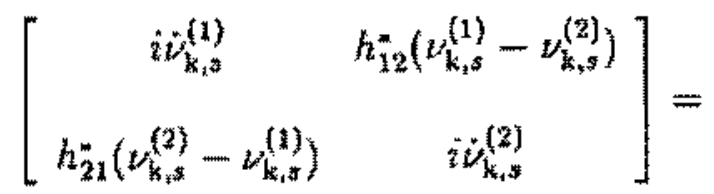

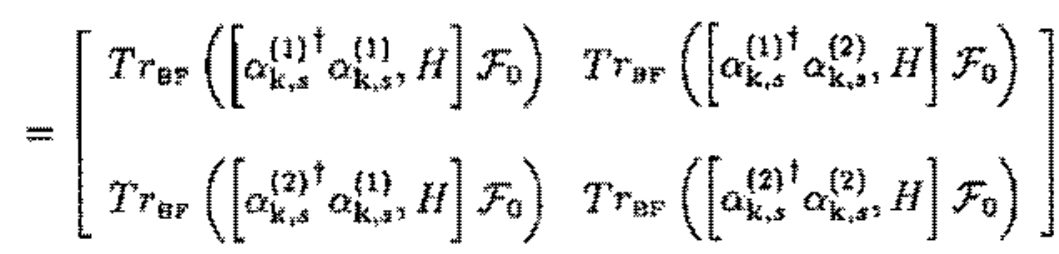

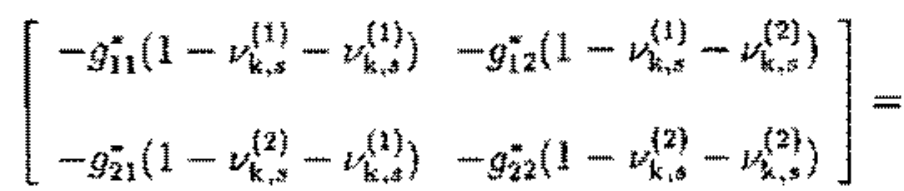

$$
\begin{aligned}
& =\left[\begin{array}{cc}
T r_{\mathrm{er}}\left(\left[\alpha_{-k, s}^{(1)} \alpha_{k, s}^{(1)}, H\right] \mathcal{F}_{0}\right) & \operatorname{Trar}\left(\left[\alpha_{-k, s}^{(1)} \alpha_{k, s}^{(2)}, H\right] \mathcal{F}_{0}\right) \\
\operatorname{Tr}_{\mathrm{EF}}\left(\left[\alpha_{-\mathrm{k}, s}^{(2)} \alpha_{k, s}^{(1)}, H\right] \mathcal{F}_{0}\right) & \operatorname{Tr}\left(\left[\alpha_{-k, s}^{(2)} \alpha_{k, s}^{(2)}, H\right] \mathcal{F}_{0}\right)
\end{array}\right],
\end{aligned}
$$

onde $t_{k, s}^{(1)}$ e $v_{k, s}^{(2)}$ são as ocupasões de quasi-férmions de Nambu e $h_{i j}$ e $g_{i j}$ são os elementos das matrizes $h_{k, s}$ e $g_{k, s}$,

$$
\begin{aligned}
& \mathrm{l}_{\mathrm{k}, 5}=-i\left(\begin{array}{cc}
\dot{U}_{11}^{*} U_{11}+\dot{V}_{21} V_{21}^{*} & 0 \\
0 & \hat{V}_{12} V_{12}^{*}+\dot{U}_{22}^{*} b_{22}
\end{array}\right) \\
& \mathrm{g}_{\mathrm{k}, \mathrm{s}}=-i\left(\begin{array}{cc}
0 & \dot{V}_{21} U_{22}^{*}+\dot{U}_{11} V_{12} \\
\dot{V}_{12} U_{11}^{*}+\dot{U}_{22}^{*} V_{21} & 0
\end{array}\right) .
\end{aligned}
$$

Para calcular os traços das equações (5.10 - 5.14) temos inicialmente que escrever a

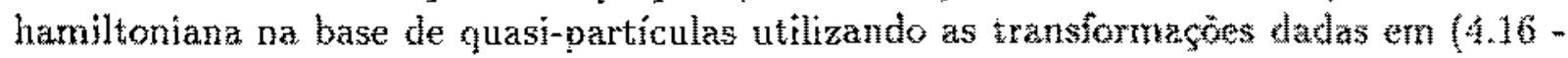
$4.17) \in(4.21)$. 
Escolhemos a seguinte reparametrizaçăo para os coeficientes $x_{\mathrm{p}}$ e $y_{\mathrm{p}}$ da transformação da Bogoliubov dos operadores bosónicos

$$
\begin{aligned}
& x_{p}=\cosh \kappa_{p}+i \frac{\eta_{p}}{2} \\
& y_{p}=\sinh \kappa_{p}+i \frac{\eta_{p}}{2}
\end{aligned}
$$

consistente com as relacoues de normalizaçăo e completeza (4.20).

No caso dos férmions, verifica-se que independente da reparametrizaço utilizada, obtém-se dinămica apenas para duas variaveis, enquanto as outras se comportam como fases globais que se simplificam. Ou seja, é necessário somente duas variàveis para dest crever a dinâmica das densidades fermionicas. Utilizaremos a seguinte reparametrização consistente com as equaçoes de unitariedade (4.24)

$$
\begin{aligned}
& U_{11}=U_{22}=\cos \varphi_{\mathrm{k}, \mathrm{s}} \\
& V_{12}=-V_{2 !}=\sin \varphi_{\mathrm{k}, s} e^{i \eta_{\mathrm{H}_{2}} s} .
\end{aligned}
$$

A partir de $(5.6-5.9)$ escrita na base de quasi-partículas podemos calcular os tracos das equaçoes $(5.10-5.12)$. Para a amplitude $B(t)$ encontramos

$\dot{E}=\frac{1}{4 \pi} \frac{\left(\mu^{2} B_{\mathrm{R}}+i \Omega^{2} B_{\mathrm{t}}\right)}{\Omega}+\frac{g}{(2 V \Omega)^{1 / 2}} \sum_{k, 4} \frac{\bar{M}}{k_{0}}\left[\cos 2 \varphi_{\mathrm{k}, s}+\frac{|k|}{M} \sin 2 \varphi_{\mathrm{k}_{\mathrm{s}}} \cos \gamma_{k, 9}\right]\left(1-\nu_{\mathrm{k}, s}^{(1)}-\nu_{\mathrm{k}, s}^{(2)}\right)$

Separando as partes real e imaginária e escrevento em termos do condensado $(\omega)$ e do momento conjugado $(\Pi)$ temos

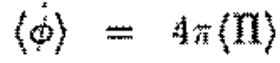

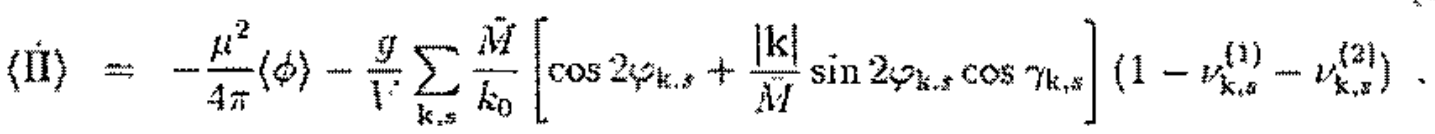


Calculando os comutadores das equaçoes $(5.11-5.12)$, com a hamiltoniana (5.6 - 5.9) eserita na base de quasi-partículas, e tomando o traço em $\mathcal{F}_{0}=\mathcal{F}_{0}^{B} \mathcal{F}_{0}^{F}$, dadas em (4.38 4.39), obtemos as equaçöes

$$
\begin{aligned}
\dot{i}_{\mathrm{p}} & =0 \\
-i \dot{\kappa}_{\mathrm{p}}+\frac{e^{-\kappa_{\mathrm{p}}}}{2}\left(\dot{\eta}_{\mathrm{p}}+\eta_{\mathrm{p}} \dot{\kappa}_{\mathrm{p}}\right) & =4 \pi\left(\mathrm{p}^{2}+\Omega^{2}\right)^{1 / 2}\left[\left(\frac{e^{2 \kappa_{\mathrm{p}}}}{2}-\frac{1}{(4 \pi)^{2}} \frac{\left(\mathrm{p}^{2}+\mu^{2}\right)}{\left(\mathrm{p}^{2}+\Omega^{2}\right)} \frac{e^{-2 \kappa_{\mathrm{p}}}}{2}\right)-\frac{\eta_{\mathrm{p}}^{2}}{2}+i \eta_{\mathrm{p}} e^{\kappa_{\mathrm{p}}}\right] .
\end{aligned}
$$

Separando as partes real e imaginária da equaçăo (5.20) temos

$$
\begin{aligned}
\dot{\kappa}_{\mathrm{p}} & =-4 \pi\left(\mathrm{p}^{2}+\Omega^{2}\right)^{1 / 2} \eta_{\mathrm{p}} e^{\alpha_{\mathrm{p}}} \\
\dot{\eta}_{\mathrm{p}} e^{-\kappa_{\mathrm{p}}} & =\left(\mathrm{p}^{2}+\Omega^{2}\right)^{1 / 2}\left[4 \pi e^{2 \kappa_{\mathrm{p}}}-\frac{1}{4 \pi} \frac{\left(\mathrm{p}^{2}+\mu^{2}\right)}{\left(\mathrm{p}^{2}+\Omega^{2}\right)} e^{-2 \alpha_{\mathrm{p}}}\right] .
\end{aligned}
$$

Analogamente, a partir de (5.6-5.9) escrita na base de quasi-partículas, podemos calcular os comutadores das equaçós cinéticas $(5.13-3.11)$ para em seguida tomar o traço ern $F_{0 .}$ Com isso obtemos

$$
\dot{u}_{k, s}^{(\lambda)}=0
$$

$$
\begin{aligned}
& \left(i \hat{\varphi}_{k, s}+\dot{\gamma}_{k, s} \sin \varphi_{k, s} \cos \varphi_{k, s}\right) e^{-s_{k, s}}=\frac{\left(k^{2}+m \bar{M}\right)}{k_{0}} \sin 2 \varphi_{k, s} e^{-i \gamma_{k, s}} \\
& +(\bar{M}-m) \frac{|\mathrm{k}|}{k_{k_{0}}}\left[\left(\cos ^{2} \varphi_{\mathrm{k}, s}-\sin ^{2} \varphi_{\mathrm{k}, \mathrm{s}} \mathrm{e}^{-2 i \eta_{\mathrm{k}, s}}\right)\right] \\
& -g \frac{\hat{M}}{k_{0}}\langle\phi\rangle\left[\sin 2 \varphi_{\mathrm{k}, s} e^{-i \gamma_{k, s}}-\frac{|\mathrm{k}|}{M}\left(\cos ^{2} \varphi_{\mathrm{k}, \mathrm{s}}-\sin ^{2} \varphi_{\mathrm{k}, \mathrm{e}} e^{-2 i \gamma_{\mathrm{k}, s}}\right)\right] \text {. }
\end{aligned}
$$


Nas equaçóes abaixo näo colocaremos $o$ índice de spin $s$ nass variáveis $\varphi_{k, s}$ e $\gamma_{k, s}$ pois as equações dinâmicas (5.17) e (5.23) dependem apenas de $\mathrm{k}$ e năo de s. Isto se deve ao fato de năo termos misturado spin na transformaçẩo de Bogoliubov dos férmions.

Separando as partes real e imaginária da eq. $(5.23)$ temos

$$
\begin{aligned}
& \dot{\rho}_{\mathrm{k}}=\frac{|\mathrm{k}|}{k_{0}}\left[\vec{M}-(m-g(\phi)) \sin \gamma_{\mathrm{k}}\right. \\
& \dot{\gamma}_{\mathrm{k}}=2 \frac{\left[\mathrm{k}^{2}+(m-g(\phi)) \bar{M}\right]}{k_{0}}+2 \frac{[\bar{M}-(m-g(\phi\rangle)]}{k_{0}}|\mathrm{k}| \cot 2 \varphi_{\mathrm{k}} \cos \gamma_{\mathrm{k}} .
\end{aligned}
$$

Observe que as equacões $(5.18),(0.79),(5.21),(5,22)$ e $(5,24)$ säo equaçò do tho cinético que descrevem a evolugão temporal das ocupacỏes de quasi-bósons, quasi-fémions e dos coeficientes de Bogoliuboy reparametrizados $k_{p}, \eta_{p}, \varphi_{\mathrm{k}}$ e $\gamma_{\mathrm{k}}$, ou seja estas equaçoes de movimento descrevem a evoluçäo temporal de todas as variáveis de um corpo.

As equaçöes de movimento na aproximaçäo de campo médio conservam a energia média do sistemá,

$$
\begin{aligned}
& \frac{\langle H\rangle}{V}=\operatorname{Tr} H \mathcal{F}_{0}
\end{aligned}
$$

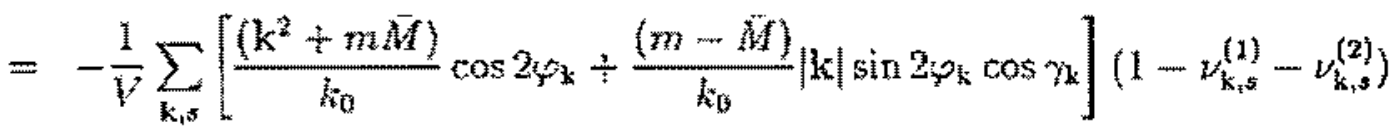

$$
\begin{aligned}
& +\frac{1}{4 \pi}\left(\frac{\mu^{2}}{2}\langle\phi\rangle^{2}+\frac{\langle\Pi\rangle^{2}}{2}\right)
\end{aligned}
$$

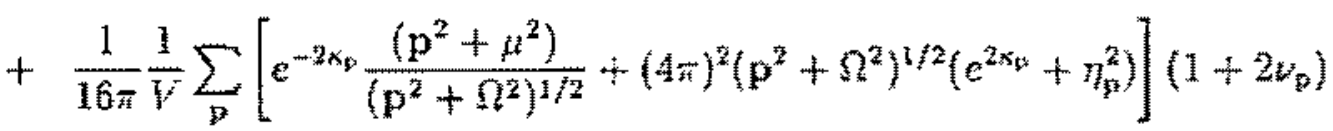

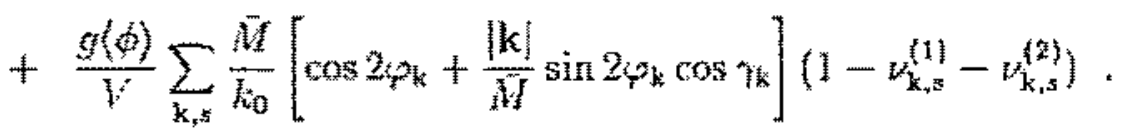

Derivando temporalmente (5.25) e substituindo as equaçöes cinéticas $(5.18),(5.19),(5.21)$, (5.22) e (5.24), podemos verificar a conservaçào de energía do sistema,

$$
\frac{d}{d t}(H)=0
$$


Estudaremos agora o estado fundamental do plasma escalar uniforme na aproximaça de campo médio. Para isto vamos considerar a soluça estacionária das equaçós cinétícas $(5.18),(5.21)$ e $(5.24)$.

\subsection{Renormalização}

As equações obtidas na seçäo anterior contèm integrais divergentes de modo que um processo de renormalizaçâo toma-se necessário. Com o objetivo de renormalizar a teoria,

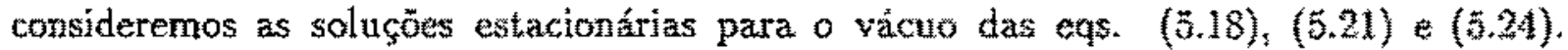
Tomando $\dot{\gamma}_{\mathrm{k}}=\dot{\varphi}_{\mathrm{k}}=\hat{t}_{\mathrm{p}}=\dot{\eta}_{\mathrm{p}}=(\dot{\phi})=(\dot{I})=0$ e $z_{\mathrm{p}}=k_{k, \mathrm{k}}=0$ nas equaçöes que descrevem a dinâmica do plasma escalar nniforme na aproximação de campo médio,

$$
\begin{aligned}
& {\left.[\bar{M}-(m-g(\omega))] \sin \gamma_{\mathrm{k}}\right|_{\mathrm{eq}}=0} \\
& \left.\cot 2 \varphi_{\mathrm{k}}\right]_{\mathrm{eq}}=-\frac{\left[\mathrm{k}^{2}+\left(m-\left.g(\phi)\right|_{\mathrm{eq}}\right) M\right]}{\left[M-\left(m-\left.g(\phi)\right|_{\mathrm{eq}}\right)\right] \mathrm{k}\left|\cos \gamma_{\mathrm{k}}\right|_{\mathrm{eq}}} \\
& \left.\eta_{\mathrm{p}}\right|_{\mathrm{rq}_{\mathrm{q}}}=0 \\
& 4 \pi\left(\mathrm{p}^{2}+\mathrm{n}^{2}\right) e^{2 \kappa}-\frac{1}{4 \pi}\left(\mathrm{p}^{2}+\hat{t}^{2}\right) e^{-2 \kappa_{\mathrm{p}}}=0 \\
& (I))_{\mathrm{r}}=0 \\
& \left.\langle\phi\rangle\right|_{\mathrm{eq}}=-\frac{4 \pi g}{V \mu^{2}} \sum_{\mathrm{k}_{\mathrm{k}} s} \frac{\ddot{M}}{k_{\mathrm{o}}}\left(\left.\cos 2 \varphi_{\mathrm{k}}\right|_{\mathrm{eq}}+\left.\left.\frac{|\mathrm{k}|}{\bar{M}} \sin 2 \varphi_{k}\right|_{\mathrm{kq}} \cos \gamma_{\mathrm{k}}\right|_{\mathrm{eq}}\right) .
\end{aligned}
$$

Primeitamente vanos estudar a relaçäo entre o parâmetro de expansâo dos campos fermionicos $\bar{M}$ e massa ma la lagrangiana. A equaçào (5.27) tem duas soluçoes

$$
\begin{gathered}
1^{*} \text { soluçào: } \quad M=m-\left.g(\phi) \Longrightarrow \tan \varphi_{\mathrm{k}}\right|_{\text {+a }}=0 \\
\text { com }\left.\sin \gamma_{k}\right|_{\text {eq }} \neq 0
\end{gathered}
$$




$$
\begin{array}{ll}
2^{\mathrm{a}} \text { soluçă } \quad & \left.\sin \gamma_{\mathrm{k}}\right|_{\mathrm{eq}}=0 \\
& \operatorname{com} \ddot{M} \neq m-g(\phi) .
\end{array}
$$

Imediatamente podemos interpretar a equação (5.33) como a soluçầ trivial do sistema. Substituindo (5.33) nu transformação de Nambu $(4.23-4.25)$, utilizando a parametrizaça (5.16), verificamos que a transformaçăo de Nambu toma-se uma matriz identidade. Ou seja, para ejta solução não há dinâmica efetiva nas variâvêt fermiônicas de Nambu.

Estudemos a segunda soluçăo. Substituindo $(5.34)$ na eq. (5.28) obtemos

$$
\begin{aligned}
& \left.\cos 2 \psi_{k}\right]_{\epsilon q}=-\frac{\left(\mathrm{k}^{2}+M M\right)}{\left[\left(\mathrm{k}^{2}+M M\right)^{2}+(M-M)^{2} \mathrm{k}^{2}\right]^{1 / 2}} \\
& \left.\sin 2 \varphi_{\mathrm{k}}\right]_{* 4}=\frac{\left.(-1)^{n}(M-M) \mid \mathrm{k}\right)}{\left[\left(\mathrm{k}^{2}+M M\right)^{2}+(M-M)^{2} \mathrm{k}^{2}\right]^{1 / 2}}
\end{aligned}
$$

onde definimos

$$
M \equiv m-g(\phi)]_{\text {特 }} \text {. }
$$

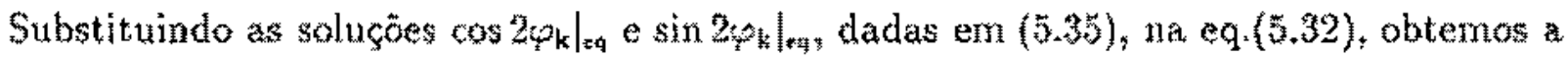
equaçào

$$
\left.\langle\phi\rangle\right|_{\mathrm{c4}}=\frac{4 \pi g}{\mu^{2}} \sum_{3} \frac{1}{(2 \pi)^{3}} \int t^{3} \mathrm{k} \frac{M}{\left(\mathrm{k}^{2}+M^{2}\right)^{1 / 2}}
$$

que contém uma integnal divergente. Observe que transformamos a soma discreta sobre os momentos em uma soma contínua azendo

$$
\sum_{\mathrm{k}} \rightarrow \frac{\mathrm{V}}{(2 \pi)^{3}} \int \mathrm{d}^{3} \mathrm{k}
$$

Caso tivéssemos escolhido o paràmetro de expansão $\bar{M}$ igual a massa $m$ da laggrangiana, obteriamos a mesma equação de gap (5.37). Portanto mostramos que para o modelo do plasma escalar existe somente uma possivel expansäo para os campos fermionicos dada 
pela massa $m$ da lagrangiana. Consequentemente, tomaremos $\bar{M}=m$ no restante do traballho.

Agora, com o objetiwo de renormalizar a teoria (eliminar suas divergencias) introdu zimos os seguintes contratermos

$$
4 \pi \mathcal{C}_{c}=\frac{A}{1 !} \phi+\frac{\delta_{i^{2}}^{2}}{2 !} \phi^{2}+\frac{C}{3 !} \phi^{3}+\frac{D}{4 !} \phi^{4}
$$

na densidade de lagrangiana (4.1) do plasma escalar. Neste caso, refazendo os cálculos com os contralemos, a equaçăo $(5.37)$ transforma-se na equaçào de gap

$$
\begin{aligned}
& \left(A+\frac{C}{4} \frac{1}{(2 \pi)^{3}} \int d^{3} \mathrm{p} \frac{\left.e^{-2 \kappa_{\mathrm{p}}}\right|_{\mathrm{Lq}}}{\left(\mathrm{p}^{2}+\Omega^{2}\right)^{1 / 2}}\right)+\left.\left(\mu^{2}+\delta \mu^{2}+\frac{D}{4} \frac{1}{(2 \pi)^{3}} \int d^{3} \mathrm{p} \frac{\left.e^{-2 \kappa_{\mathrm{p}}}\right|_{\mathrm{eq}}}{\left(\mathrm{p}^{2}+\Omega^{2}\right)^{1 / 2}}\right)\langle\phi\rangle\right|_{\mathrm{Cq}}+ \\
+ & \frac{C}{2}\left\langle\left.\phi\right|_{\mathrm{eq}} ^{2} \div \frac{D}{6}\left\langle\left.\phi\right|_{\mathrm{eq}} ^{3}=-8 \pi g \frac{1}{(2 \pi)^{3}} \int d^{3} \mathrm{k} \frac{M}{\left(\mathrm{k}^{2}+M^{2}\right)^{1 / 2}}\right.\right.
\end{aligned}
$$

onde $M$ é definida em (5.36). É conveniente fazer a mudança de variável

$$
\frac{1}{\left.\left(\mathrm{p}^{2}+\Omega_{\mathrm{p}}^{2}\right)_{\mathrm{w}}\right)^{1 / 2}}=\frac{1}{4 \pi} \frac{\left.e^{-2 \kappa_{p}}\right)_{\infty}}{\left(\mathrm{p}^{2}+\Omega^{2}\right)^{1 / 2}}
$$

Dessa forma, a nova variảvel dinâmica $\Omega_{p}^{2}$ pode ser interpretada como variável dinâmica no sentido de massa efetiva para o modo de momento $p$. Veste caso, as equaçoes (5.29) e (5.30) com os contratermos no equillorio säo datas por

$$
\begin{aligned}
& \left.\eta_{p}\right|_{\text {es }}=0
\end{aligned}
$$

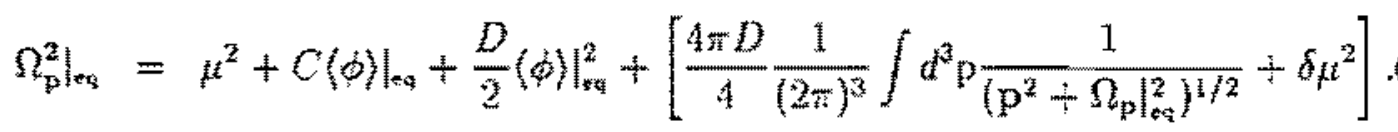

Neste ponto fxamos o parandro de expáns em (4.3). como sendo a massa efetiva $\left.\Omega_{\mathrm{p}}\right|_{\mathrm{kq}}$ dada em $(5,42)$. observando que no equilibrio esta variáyel dinânica independe de $p$, isto é, 


$$
\Omega^{2}=\mu^{2}+\left.C\langle\phi\rangle\right|_{\mathrm{eq}}+\left.\frac{D}{2}\langle\phi\rangle\right|_{* q} ^{2}+\left[\frac{4 \pi D}{4} \frac{1}{(2 \pi)^{3}} \int d^{3} \mathrm{p} \frac{1}{\left(p^{2}+\Omega^{2}\right)^{1 / 2}}+\delta \mu^{2}\right] .
$$

Portanto, no caso estático o fato da massa efetiva bosônica $\Omega_{p}$, definida em $(5.40)_{2}$, independer do momento $p_{2}$ implica que temos parâmetros a mais en nossa teoria, o que justifica termos fixado o parămetro de expansăo $\Omega=\left.\Omega_{\mathrm{p}}\right|_{\text {eq }}$ na equaçào aç̉ma. Obviamente, to caso dinâmico este procedimento năo é possivel, já que a massa efetiva $\Omega_{p}$ depende do momento.

Resta-nos ajustar os coeficientes $A, \delta \mu^{2}{ }_{2} C$ e $D$ de modo a absorver as divergências através de uma redefinição dos parămetros da densidade de lagrangiana (4.1). Fazendo isto, os contratermos que renormalizam a equação de gap (5.39) e a energia sâo dados por

$$
\begin{aligned}
D & =48 \pi g^{4} L(m) \\
\delta \mu^{2} & =-96 \pi^{2} g^{4} L(m) G(\mu)-16 \pi g^{2} G(0)+24 \pi m^{2} g^{2} L(m) \\
C & =-48 \pi m g^{3} L(m) \\
A & =96 \pi^{2} m g^{3} L(m) G(\mu)+16 \pi m g\left(0(0)-8 \pi m^{3} g L(m)\right.
\end{aligned}
$$

onde

$$
\begin{aligned}
& L(m) \equiv \frac{1}{(2 \pi)^{3}} \int d^{3} \mathrm{k} \frac{1}{2 \mathrm{k}^{2}\left(\mathrm{k}^{2}+m^{2}\right)^{1 / 2}} \\
& G(m) \equiv \frac{1}{(2 \pi)^{3}} \int d^{3} \mathrm{k} \frac{1}{2\left(\mathrm{k}^{2}+m^{2}\right)^{1 / 2}} \\
& G(\mu) \equiv \frac{1}{(2 \pi)^{3}} \int d^{3} \mathrm{p} \frac{1}{2\left(\mathrm{p}^{2}+\mu^{2}\right)^{1 / 2}}
\end{aligned}
$$

Substituindo A e $C$ no primeiro termo da equaçào (5.39) temos 


$$
\left(A+\frac{4 \pi C}{4} \frac{1}{(2 \pi)^{3}} \int d^{3} p \frac{1}{\left(p^{2}+\Omega^{2}\right)^{1 / 2}}\right)=16 \pi m g G(m)+24 \pi m g^{3} L(m)[G(u)-G(\Omega)]
$$

onde verificamos que o primeiro termo cancela uma parte da divergência do lado direito da equação (5.39). O segundo termo deve se anular para que a equação (5.39) seja finita, de modo que escolhemos o parâmetro de expansäo do campo bosônico $\Omega=\mu$. Ou seja, a equação de gap (5.39) e o esquema de renomalização exigem que no equilíbrio o paràmetro de expansāo $\Omega$ seja igual a massa $\mu$ da lagrangiana,

$$
\frac{1}{\left(p^{2}+\Omega^{2}\right)}=\frac{1}{\left(p^{2}+\left.\Omega_{p}\right|_{e q} ^{2}\right)^{1 / 2}}=\frac{e^{-2 x_{p} l_{e q}}}{\left(p^{2}+\Omega^{2}\right)^{1 / 2}} \rightarrow \frac{1}{\left(p^{2}+u^{2}\right)^{1 / 2}}
$$

0 que implica em $\kappa_{p}=0$. Isto quer dizer que no equilibrio as equaçós para as variáveis bosônicas são $\eta_{p}=0$ e $\kappa_{p}=0$. Em outras palavras, os termos

$$
\frac{1}{4 \pi} \frac{C}{3 !} \theta^{3}+\frac{1}{4 \pi} \frac{D}{4 !} t^{4}
$$

săo necessários para a renormalizaçâo da teoria, mas nầ geram dinâmica para as variáveis de emparelhamento bosonicas. Portanto, daqui em diante, nào consideraremos mais as equaz̧o ô das variaveis de emparelhamento bosônicas.

Voltemos agora a equaça de gop para os férmions (5.39). Substituindo $A, \delta \mu^{2}, C$ e $D$ dados em $(5.44-5.47)$ nos outros termos da eq. $(5.39)$, temos

$$
\begin{aligned}
& \left(-3 \pi m g \frac{1}{(2 \pi)^{3}} \int d^{3} \mathrm{k} \frac{1}{\left(\mathrm{k}^{2}+m^{2}\right)^{1 / 2}}\right) \\
+ & \left.\left(h^{2}-8 \pi g^{2} \frac{1}{(2 \pi)^{3}} \int d^{3} \mathrm{k} \frac{1}{\mathrm{k}}+12 \pi m^{2} g^{2} \frac{1}{(2 \pi)^{3}} \int d \mathrm{k}^{3} \frac{1}{\mathrm{k}^{2}\left(\mathrm{k}^{2}+m^{2}\right)^{1 / 2}}\right)(\phi)\right|_{\mathrm{eq}} \\
- & \left(\left.2 \pi m g^{3} \frac{1}{(2 \pi)^{3}} \int d^{3} \mathrm{k} \frac{1}{\mathrm{k}^{2}\left(\mathrm{k}^{2}+m^{2}\right)^{1 / 2}}(0)\right|_{\mathrm{eq}}+\left.4 \pi g^{4} \frac{1}{(2 \pi)^{3}} \int d^{3} \mathrm{k} \frac{1}{\mathrm{k}^{2}\left(\mathrm{k}^{2}+m^{2}\right)^{1 / 2}}(\phi)\right|_{\mathrm{eq}} ^{3}=\right. \\
= & 8 \pi g \frac{1}{(2 \pi)^{3}} \int d^{3} \mathrm{k} \frac{M^{2}}{\left(\mathrm{k}^{2}+M^{2}\right)^{1 / 2}} .
\end{aligned}
$$


Calculando as integrais obtemos a equaçâo de gap renormalizada

$$
\left.\frac{\pi}{2} \mu^{2}\langle\phi\rangle\right|_{\log }-g M^{3}\left[\ln \left(\frac{M}{m}\right)+\frac{1}{2}\right]=0
$$

Reagrupando os resultados, a situação de equilíbrio do vácuo do plasma escalar è dada pelas equações finitas

$$
\begin{aligned}
& \left.\sin \gamma_{k}\right|_{\kappa q}=0 \\
& \left.\cot 2 \varphi_{\mathrm{k}}\right|_{ \pm q}=-\frac{\left(\mathrm{k}^{2}+M m\right)}{|\mathrm{k}|(m-M)} \\
& \left.\eta_{p}\right|_{\text {eq }}=0 \\
& \Omega_{\mathrm{p}} \mathrm{L}_{\mathrm{c}}=\Omega=\mu \\
& \langle\Pi\rangle_{e q}=0 \\
& \frac{\pi}{2} m^{2}\left(\phi||_{* 4}-g M^{3}\left[\ln \left(\frac{M}{m}\right)+\frac{1}{2}\right]=0\right.
\end{aligned}
$$

Finalmente, a energia do vácuo de nosso sistema, incluindo os contratemos que rew nornalizam a teoria, é dada por

$$
\begin{aligned}
& \frac{(H)}{V}=\left.\left(\frac{A}{4 \pi}+\frac{C}{4} \frac{1}{(2 \pi)^{3}} \int t^{3} \mathrm{p} \frac{1}{\left(\mathrm{p}^{2}+\mu^{2}\right)^{1 / 2}}\right)(\phi)\right|_{\text {eq }}
\end{aligned}
$$

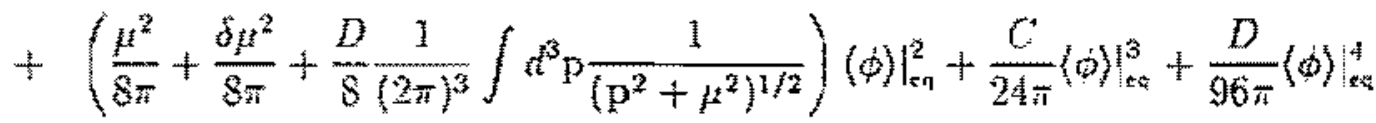

$$
\begin{aligned}
& +\frac{1}{8 \pi} \frac{1}{(2 \pi)^{3}} \int d^{3} \mathrm{p}\left(\mathrm{p}^{2}+\mu^{2}\right)^{1 / 2}+\frac{2}{(2 \pi)^{3}} \int d^{3} \mathrm{k}\left(\mathrm{k}^{2}+M^{2}\right)^{1 / 2} \\
& +\left[\frac{\pi D}{s} \frac{1}{(2 \pi)^{3}} \int d^{3} \mathrm{p} \frac{1}{\left(\mathrm{p}^{2}+\mu^{2}\right)^{1 / 2}}+\frac{\partial \mu^{2}}{4}\right] \frac{1}{(2 \pi)^{3}} \int d^{3} \mathrm{p} \frac{1}{\left(\mathrm{p}^{2}+\mu^{2}\right)^{1 / 2}} \text {. }
\end{aligned}
$$


Tomando $\langle\phi\rangle=0$, obtemos

$$
\begin{aligned}
\left.\frac{\langle H\rangle}{V}\right|_{\langle\varphi\rangle=0} & =\frac{1}{8 \pi} \frac{1}{(2 \pi)^{3}} \int d^{3} \mathrm{p}\left(\mathrm{p}^{2}+\mu^{2}\right)^{1 / 2}+\frac{2}{(2 \pi)^{3}} \int d^{3} \mathrm{k}\left(\mathrm{k}^{2}+m^{2}\right)^{1 / 2} \\
& +\left[\frac{\pi D}{8} \frac{1}{(2 \pi)^{3}} \int d^{3} \mathrm{p} \frac{1}{\left(\mathrm{p}^{2}+\mu^{2}\right)^{1 / 2}}+\frac{\delta \mu^{2}}{4}\right] \frac{1}{(2 \pi)^{3}} \int d^{3} \mathrm{p} \frac{1}{\left(\mathrm{p}^{2}+\mu^{2}\right)^{1 / 2}}
\end{aligned}
$$

que pode ser interpretada como a energia de ponto zero do vácuo. Portanto, subtraindo e somando $\frac{2}{(2 \pi)^{3}} \int d^{3} k\left(k^{2}+m^{2}\right)^{1 / 2}$ na equação (5.59), temos

$$
\begin{aligned}
& \frac{\langle H\rangle}{V}=\left.\left(\frac{A}{4 \pi}+\frac{G}{4} \frac{1}{(2 \pi)^{3}} \int d^{a} \mathrm{p} \frac{1}{\left(\mathrm{p}^{2}+h^{2}\right)^{1 / 2}}\right)(\phi)\right|_{\text {eq }} \\
& +\left.\left(\frac{\mu^{2}}{8 \pi}+\frac{\delta \mu^{2}}{8 \pi}+\frac{D}{8} \frac{1}{(2 \pi)^{3}} \int \mu^{3} \mathrm{p} \frac{1}{\left(\mathrm{p}^{2}+\mu^{2}\right)^{1 / 2}}\right)(\omega)\right|_{\mathrm{sq}} ^{2}+\left.\frac{C}{24 \pi}(\phi)\right|_{\mathrm{kq}} ^{3}+\left.\frac{D}{96 \pi}(\phi)\right|_{\mathrm{Q}} ^{4} \\
& +\frac{2}{(2 \pi)^{3}} \int d^{3} \mathrm{k}\left(\mathrm{k}^{2}+M^{2}\right)^{1 / 2}-\frac{2}{(2 m)^{3}} \int d^{3} \mathrm{k}\left(\mathrm{k}^{2}+m^{2}\right)^{1 / 2} \\
& +\frac{1}{8 \pi} \frac{1}{(2 \pi)^{3}} \int h^{a} p\left(p^{2}+n^{2}\right)^{1 / 2}+\frac{2}{(2 \pi)^{3}} \int k^{3} k\left(k^{2}+m^{2}\right)^{1 / 2} \\
& +\left[\frac{\pi D}{8} \frac{1}{(2 \pi)^{3}} \int d^{3} \mathrm{p} \frac{1}{\left(\mathrm{p}^{2}+\mu^{2}\right)^{1 / 2}}+\frac{\delta \mu^{2}}{4}\right] \frac{1}{(2 \pi)^{3}} \int d^{3} \mathrm{p} \frac{1}{\left(\mathrm{p}^{2}+\mu^{2}\right)^{1 / 2}}
\end{aligned}
$$

onde os três áltimos termos de (艹).6l) correspondem a energia de ponto zero do vácuo, de modo que redefinimos o zero da escala de energia subtraindo a densidade da energia do văcuo,

$$
\begin{aligned}
\frac{\langle H\rangle}{V} & =\left.\left(\frac{A}{4 \pi}+\frac{C}{4} \frac{1}{(2 \pi)^{3}} \int d^{3} \mathrm{p} \frac{1}{\left(\mathrm{p}^{2}+\mu^{2}\right)^{1 / 2}}\right)\langle\phi\rangle\right|_{\mathrm{eq}} \\
& \left.+\left.\left(\frac{\mu^{2}}{8 \pi}+\frac{\delta \mu^{2}}{8 \pi}+\frac{D}{8} \frac{1}{(2 \pi)^{3}} \int d^{3} \mathrm{p} \frac{1}{\left(\mathrm{p}^{2}+\mu^{2}\right)^{1 / 2}}\right)\langle\phi\rangle\right|_{\mathrm{tq}} ^{2}+\frac{C}{24 \pi}\langle\phi\rangle\right\rangle_{\mathrm{*}}^{3}+\left.\frac{D}{96 \pi}(\phi)\right|_{q q} ^{4}
\end{aligned}
$$




$$
+\frac{2}{(2 \pi)^{3}} \int d^{3} k\left(\mathrm{k}^{2}+M^{2}\right)^{1 / 2}-\frac{2}{(2 \pi)^{3}} \int d^{3} k\left(\mathrm{k}^{2}+m^{2}\right)^{1 / 2}
$$

Substituindo os contratermos $(5.44-5.47)$ an $(5.62)$ e calculando as integrais, obtemos finalmente a energia de campo médio renormalizada

$$
\frac{(H)}{V}=\frac{1}{8 \pi^{2}}\left[\frac{\pi \mu^{2}}{g^{2}}(m-M)^{2}+M^{4} \ln \left(\frac{M}{m}\right)+\frac{\left(M^{4}-m^{4}\right)}{4}\right]
$$

Portanto ${ }_{1}$ os contratermos dados em $(5.44-3.47)$ tornam finitas todas as quantidades de equilibrio de nosso sistema. Definindo $\left.\equiv g(\omega)\right|_{* a} / m$ e $E(x) \equiv\left(8 \pi^{2} / m^{4}\right)(H) / V$ na equaçăo $(5.63)$ obtemos

$$
E(x)=\frac{\pi u^{2}}{g^{2} m^{2}} x^{3}+(1-x)^{4}\left[\ln (1-x)+\frac{1}{4}\right]-\frac{1}{4}
$$

A equação de gap (5.58) pode ser reescrita como

$$
\frac{\pi \mu^{2}}{2 d^{2} m^{2}} x-(1-x)^{3}\left[\ln (1-x)+\frac{1}{2}\right]=0
$$

O comportamento da energia do estado fundamental do sistema ern função de $\left.\langle\phi\rangle\right|_{\text {eq }} \dot{e}$ mostrado na Figura 5.1 para alguns valores de $g$ e $\mathrm{m} / \mu$. 

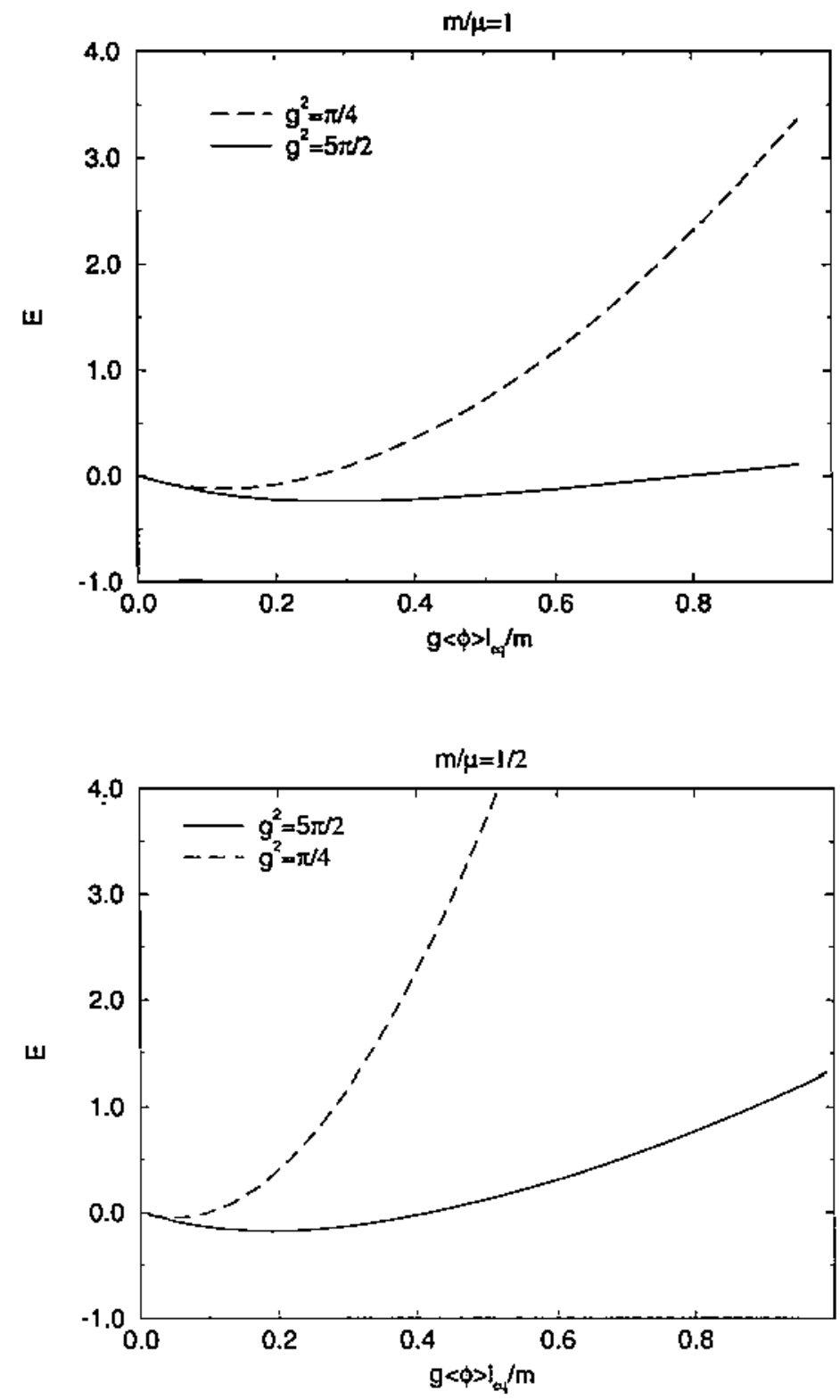

Figura 5.1: Gráfico da energia em função de $\left.g\langle\phi\rangle\right|_{\text {eq }}$ para $m / \mu=1,1 / 2$ e $g^{2}=\pi / 4,5 \pi / 2$. 
Tendo obtido a situaça de equilibrio para o estado fundamental do vắcuo do plasma escalar, dada pelas equaços (5.53 - 5.58), e sua energia de equilitrio dada em (5.63), estudaremos no capitulo seguinte a dinâmica deste sistema. 


\section{Capítulo 6}

\section{Linearização da Dinâmica de Campo Médio}

Neste capitulo obteremos a dinâmica de pequenas oscilacôes em torno da solução estacionária. Devido a semelhança destas equaçöes linearizadas com a equaçäto de LippmannSchwinger, estudaremos a fisica de quasi-equilibrio de nosso sistema através de uma analogia com a Teoria de Espaltamento, obtendo analitjcamente as amplitudes efrequência de oscilaça do sistema. Finalmente, a partir dos polos da matriz $T$ analisaremos a existência de estados ligados de quasi-férmions neste regime.

\subsection{Linearização das Equações Cinéticas}

No caso dinâmico devemos introduzir os seguintes contratermos para renormalizar a teonia

$$
4 \pi \mathcal{L}_{\mathrm{C}}=\frac{A}{1 !} \phi+\frac{\delta \mu^{2}}{2 !} \phi^{2}+\frac{C}{3 !} \phi^{3}+\frac{D}{4 !} \phi^{A}-\frac{Z}{2}(\partial \phi)^{2}
$$

onde acrescentamos à lagrangiana de contratermos do caso estático (5.38). um terno de renormalizaçāo da função de onda. En [23] J. Dias Alonso e R. Hakim moxtmm que os contratermos dados em (6.1) renomalizarn as equaçós que descrevem o regime de pequenas oscilaços em torno do equilibrio para o sistema do plasma escalat. Refazendo os callulos do capítulo 5 com os contratermos dados em (6.1), obtemos para a dirtumica efetiva do vácuo do plasma escalar na aproximaça de campo médio as seguintes equaçoes de movimento 


$$
\begin{aligned}
\dot{\varphi}_{\mathrm{k}} & =g(\phi) \frac{|\mathrm{k}|}{k_{0}} \sin \gamma_{\mathrm{k}} \\
\dot{\psi_{\mathrm{k}}} & =2 k_{0}-2 g m\langle\phi\rangle \frac{1}{k_{0}}\left(1-\frac{|\mathrm{k}|}{m} \cot 2 \varphi_{\mathrm{k}} \cos \gamma_{\mathrm{k}}\right) \\
\langle\dot{\phi}\rangle & =\frac{4 \pi}{(1+Z)}\langle\mathrm{I}\rangle \\
(\mathrm{II}) & =-\left(\frac{A}{4 \pi}+\frac{C}{2} G(\mu)\right)-\left(\frac{\mu^{2}}{4 \pi}+\frac{\delta \mu^{2}}{4 \pi}+\frac{D}{2} G(\mu)\right)\langle\phi\rangle \\
& -\frac{C}{8 \pi}\langle\phi\rangle^{2}-\frac{D}{24 \pi}\langle\phi\rangle^{3}-\frac{2 g}{(2 \pi)^{3}}\left[I_{1}(m)+I_{2}(m)\right]
\end{aligned}
$$

onde as integrais divergentes $G(\mu), I_{1}(m)$ e $I_{2}(m)$ săo daclas por

$$
\begin{aligned}
G(\mu) & =\frac{1}{(2 \pi)^{3}} \int d^{3} \mathrm{p} \frac{1}{2\left(\mathrm{p}^{2}+\mu^{2}\right)^{1 / 2}} \\
I_{1}(m) & =\int d^{3} \mathrm{k} \frac{m}{\left(\mathrm{k}^{2}+m^{2}\right)^{1 / 2}} \cos 2 \varphi_{\mathrm{k}} \\
I_{2}(m) & =\int d^{3} \mathrm{k} \frac{|\mathrm{k}|}{\left(\mathrm{k}^{2}+m^{2}\right)^{1 / 2}} \sin 2 \varphi_{\mathrm{k}} \cos \gamma_{\mathrm{k}}
\end{aligned}
$$

Primeiramente observamos que as equaçōes dinämicas acima se reduzem às equaçóes $(5.53-5.58)$ no regime estático, 0 que mostra que realmente o contratermo $-\frac{Z}{2}(\partial \phi)^{2}$ não é necessário neste caso. Verificamos tămbém, consistentemente com a discussão realizada no capítulo anterior durante o processo de renormalizaçäo [veja equaçoes (5.48 - 5.50)], que nảo há dinamica para as variáveis de emparelhamento bosônicas $\eta_{\mathrm{p}}$ e $\Omega_{\mathrm{p}}$ no caso de um sistema relativístico descrito pelo modelo do plasma escalar. Enfim, as equaçỏes cinéticas acima forman um sistema complicado nào linear de equaçöes diferenciais acopladas de modo que somente soluçōes numéricas săo possiveis.

Calcularemos a dinâmica efetiva de $\mathcal{R}^{r}$ no regime de pequenas oscilações em torno do equilibrio. As equaçòes cinéticas obtidas neste regime säo conhecidas como equações de campo médio linearizadas e são equivalentes às equaçöes de RPA [36]. Veremos que será possivel obter soluçōes analíticas para a dinâmica das variáveis fermionticas de nosso sistema neste regime. 
Com o objetivo de estudar o regime de pequenas oscilacöes en torno do equilíbrio, vamos inicialmente expandir as varižveis dinâmicas fermionnicas e do condensado até primeira ordern em torno das soluções estacionárias

$$
\begin{aligned}
& \phi_{k}=\phi_{\mathrm{k}}^{(0)}+\delta \phi_{\mathrm{k}} \\
& \gamma_{\mathrm{k}}=\gamma_{\mathrm{k}}^{(0)}+\delta \gamma_{\mathrm{k}} \\
& (\phi)=\left(\omega^{(0)}+\delta(\phi)\right. \\
& (\Pi)=(\Pi)^{(0)}+\delta(\Pi)
\end{aligned}
$$

onde $\varphi_{\mathrm{k}}^{(0)}=\left.\varphi_{\mathrm{k}}\right|_{\mathrm{eq}}, \gamma_{\mathrm{k}}^{(0)}=\left.\gamma_{\mathrm{k}}\right|_{\mathrm{eq}},\langle\phi\rangle^{(0)}=\left.\langle\phi\rangle\right|_{\mathrm{kq}} \mathrm{e}(\Pi\rangle^{(0)}=\left.\langle\Pi\rangle\right|_{\mathrm{eq}}$ săo as soluçōes estacionávias de equilíbrio, enquanto $\delta \varphi_{k}, \delta \gamma_{k}, \delta\langle\phi\rangle$ e $\delta\langle\Pi\rangle$ sz̃o pequenas oscilações em torno das soluçōes de equilíbrio.

Devemos notar que temos infinitas soluçôes estacionatrias para as variáveis fermionicas devido ao fator $n$ [veja eq. (5.35)]. Isto se deve ao fato de utilizarmos variáveis do tipo ângulo-açào de modo que estes infinitos pontos de equilíbrio são equivalentes. Tomemos portanto $n=0$. As soluçoes de equilíbrio sấo então dadas por

$$
\begin{aligned}
\cos 2 \varphi_{\mathrm{k}}^{(0)} & =-\frac{\left[\left(\mathrm{k}^{2}+m^{2}\right)-m g(\phi)^{(0)}\right]}{\left(\mathrm{k}^{2}+m^{2}\right)^{1 / 2}\left(\mathrm{k}^{2}+M^{2}\right)^{1 / 2}} \\
\sin 2 \varphi_{k}^{(0)} & =\frac{\left.g(\phi)^{(0)} \mid \mathrm{k}\right)}{\left(\mathrm{k}^{2}+m^{2}\right)^{1 / 2}\left(\mathrm{k}^{2}+M^{2}\right)^{1 / 2}} \\
m^{(0)} & =0 \\
(\Pi)^{(0)} & =0 \\
\frac{m \mu^{2}}{2}(\phi)^{(0)} & =g M^{*}\left[\ln \left(\frac{M}{m}\right)+\frac{1}{2}\right] .
\end{aligned}
$$


Para linearizar as equaçöes $(6.2-6.5)$ temos que expandir algumas funções de $\varphi_{k}$ e $\gamma_{k}$ em torno do equilíbrio até primeira ordem. Expandindo as funçóes de $\gamma_{k}$ temos

$$
\begin{aligned}
& \sin \gamma_{k}=\sin \gamma_{k}^{(0)}+\cos \gamma_{k}^{(0)} \delta \gamma_{k}+\mathcal{O}\left(\delta \gamma_{k}\right)^{2}=\delta \gamma_{k} \\
& \cos \gamma_{k}=\cos \gamma_{k}^{(0)}-\sin \gamma_{k}^{(0)}+\delta\left(\delta \gamma_{k}\right)^{2}=1
\end{aligned}
$$

e para $\varphi_{\mathrm{k}}$ temos

$$
\begin{aligned}
& \sin 2 \varphi_{k}=\sin 2 \varphi_{k}^{(0)}+2 \cos 2 \varphi_{k}^{(0)} d \varphi_{k}+O\left(\delta \varphi_{k}\right)^{2} \\
& \cos 2 y_{k}=\cos 2 \gamma_{k}(0)-2 \sin 2 \varphi_{k}^{(0)} b_{p_{k}}+\phi\left(\delta p_{k}\right)^{2} .
\end{aligned}
$$

As integrais divergentes $l_{1}(m)$ e $l_{2}(m)$ também devem ser expandidas uma vez que são funçóes de $\varphi_{k}$ e $\gamma_{k}$,

$$
\begin{aligned}
& I_{1}=I_{1}^{(0)}+I_{1}^{(1)}+\mathcal{O}\left(\hat{b}_{\mathrm{p}}\right)^{2}
\end{aligned}
$$

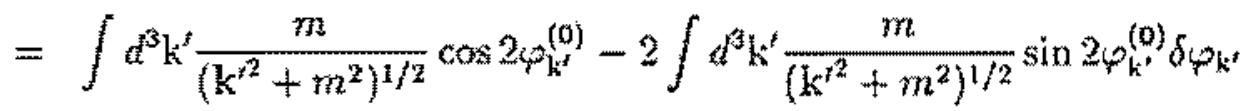

$$
\begin{aligned}
& I_{2}=I_{2}^{(0)}+I_{2}^{[1]}+\mathcal{O}\left(\delta \psi_{\mathrm{k}}^{2}, \delta \gamma_{\mathrm{k}}^{2}, \delta \varphi_{\mathrm{k}}, \delta \gamma_{\mathrm{k}}\right) \\
& =\int d^{3} \mathrm{k}^{\prime} \frac{\left|\mathrm{k}^{\prime}\right|}{\left(\mathrm{k}^{\prime^{2}}+m^{2}\right)^{1 / 2}} \sin 2 \varphi_{\mathrm{k}^{\prime}}^{(0)} \\
& +2 \int d^{3} k^{\prime} \frac{\left|k^{\prime}\right|}{\left(k^{\prime 2}+m^{2}\right)^{1 / 2}}\left(\cos 2 \varphi_{k^{\prime}}^{(0)} \delta \varphi_{k^{3}}-\sin 2 \varphi_{k}^{(0)} \sin \eta_{k}^{(0)} b_{k}\right)
\end{aligned}
$$

Substituindo as formas linearizadas acima nas equaçös $(6.2-6.5)$ obtemos a formetinearizada destas equaçôes cinéticas 


$$
\begin{aligned}
& \delta_{\varphi_{\mathrm{k}}}=g\langle\phi\rangle^{(0)} \frac{|\mathrm{k}|}{k_{0}} \delta \gamma_{\mathrm{k}} \\
& g(\phi)^{(0)}|\mathrm{k}| \delta \gamma_{\mathrm{k}}=-4 k_{0}\left(\mathrm{k}^{2}+M^{2}\right) \delta \varphi_{\mathrm{k}}-2 g|\mathrm{k}| k_{0} \delta\langle\phi\rangle \\
& \delta(\dot{\phi})=\frac{4 \pi}{(1+Z)} \delta(I I) \\
& \delta(\dot{\Pi})=-\left(\frac{\mu^{2}}{4_{\pi}}+\frac{\delta \mu^{2}}{4 \pi}+\frac{D}{2} G(\mu)\right) \delta(\phi) \\
& -\frac{C}{4 \pi}\langle\phi\rangle^{(0)} \delta\langle\phi\rangle-\frac{D}{8 \pi}\langle\phi)^{(0)^{2}} \delta\langle\phi\rangle+\frac{4 g}{(2 \pi)^{3}} \int d^{3} k^{\prime} \frac{\left|k^{2}\right|}{\left(k^{2^{2}}+M^{2}\right)^{1 / 2}} \delta \phi_{\mathrm{k}} .
\end{aligned}
$$

Observamos que as equaçòes de campo médjo linearizadas $(6.14-6.17)$ sào homogêneas com coeficientes que năo dependem explicitamente do tempo. Logo, as suas soluçōes são do tipo

$$
\begin{aligned}
& \delta \varphi_{\mathrm{k}}=\Phi_{\mathrm{k}} e^{i \omega t} \\
& \delta \gamma_{\mathrm{k}}=\Gamma_{\mathrm{k}} e^{i \omega t} \\
& \delta\langle\phi\rangle=\Phi e^{i \omega t} \\
& \delta\langle\Pi\rangle=\Pi e^{i \omega t},
\end{aligned}
$$

onde $\omega$ é a oscilação do emparelhamento em tomo do equilíbrio e $\Psi_{k}, \Gamma_{k}, \Phi$ e II são as amplitudes de pequenas oscilaçöes. Substituindo as soluçōes (6.15) em (6.14 - 6.17), obtemos equaçoses para as amplitudes de oscilação $\Psi_{k}, \Gamma_{k}$, $\Phi$ e $\Pi$,

$$
\begin{aligned}
i \omega \Psi_{k} & =g\langle\partial\rangle^{(0)} \frac{|\mathrm{k}|}{k_{0}} \Gamma_{k} \\
i \omega g\langle\phi\rangle^{(0)}|\mathrm{k}| \Gamma_{k} & =-4 k_{0}\left(\mathrm{k}^{2}+M^{2}\right) \Psi_{k}-2 g|k| k_{0} \Phi
\end{aligned}
$$




$$
\begin{aligned}
i \omega \Phi & =\frac{4 \pi}{(1+Z)} \Pi \\
i \omega \Pi & =-\left(\frac{\mu^{2}}{4 \pi}+\frac{\delta \mu^{2}}{4 \pi}+\frac{D}{2} G(\mu)\right) \Phi \\
& -\frac{C}{4 \pi}\langle\phi\rangle^{(0)} \Phi-\frac{D}{8 \pi}\langle\phi\rangle^{(0)^{2}} \Phi+4 g \frac{1}{(2 \pi)^{3}} \int d^{3} \mathrm{k}^{\prime} \frac{\left|\mathrm{k}^{\prime}\right|}{\left(\mathrm{k}^{\prime 2}+M^{2}\right)^{1 / 2}} \Psi_{\mathrm{k}^{\prime}}
\end{aligned}
$$

Na seção seguinte resslveremos analiticamente este sistema de equaçōes acopladas para as amplitudes de oscilação através de uma analogia corn a Teoria de Espalhamento.

\subsection{Solução das Equações Cinéticas de Campo Médio Linearizadas}

Nesta seção vamos resolver analiticamente o sistema de equaşos acopladas $(6.19 \cdot 6.22)$, as quais descrevem a dinămica efetiva de pequenas oscilaçoes do vácuo do plasma escalar em torno da soluçăo estacionária na aproximação de campo médio. Para isto faremos uma analogia com a Teoria de lispalhamento, já que as equaçóe linearizadas obtidas são semelhantes a equaçăo de Lippmann-Schwinger. A partir deste analogia, reinterpretamos o nosso problema de pequenas oscilaçòes do emparelhamento, utilizando o teorema de Thouless [31], o qual diz que a uma perturbação linear corresponde um par emparelhado de quasi-partículas em adiçâo ao vácuo gaussiano, como um problema de espalhamento de quasi-férmions gerados da flutuaçẵo do vácuo.

Substituindo a equaçào $(6.20) \mathrm{cm}(6.19)$ temos

$$
-\omega^{2} \Psi_{\mathrm{k}}=-4\left(\mathrm{k}^{2}+M^{2}\right) \Psi_{\mathrm{k}}-2 g|\mathrm{k}| \Phi
$$

e substituindo (6.22) em (6.21) obtemos

$$
\begin{aligned}
(1+Z) \omega^{2} \Phi & =\left(\mu^{2}+\delta \mu^{2}+\frac{D}{4} \frac{1}{(2 \pi)^{3}} \int d^{3} \mathrm{p} \frac{1}{\left(\mathrm{p}^{2}+\mu^{2}\right)^{1 / 2}}\right) \Phi+C\langle\phi\rangle^{(0)} \Phi \\
& +\frac{D}{2}\langle\phi\rangle^{(0)^{2}} \Phi-16 \pi g \frac{1}{(2 \pi)^{3}} \int d^{3} \mathrm{k}^{3} \frac{\left|\mathrm{k}^{\prime}\right|}{\left(\mathrm{k}^{z^{2}}+M^{3}\right)^{1 / 2}} \Psi_{\mathrm{k}^{t}}
\end{aligned}
$$


Finalmente, substituindo $\Phi$ da equação (6.23) em (6.24) obtemos a equaçäo para a amplitude de oscilaçäo $w_{k}$

$$
\left[(1+2) \omega^{2}-N\right] \frac{\left[\omega^{2}-4\left(k^{2}+M^{2}\right)\right]}{2 g|k|} \Psi_{k}=-16 \pi g \frac{1}{(2 \pi)^{3}} \int d^{3} k^{\prime} \frac{|k|}{\left(k^{2}+M^{2}\right)^{1 / 2}} \Psi_{k^{*}}
$$

orde

$$
N=\left(\mu^{2}+\partial_{n^{2}}^{2}+\frac{D}{2} 4 \pi G(\mu)\right)+C(\varphi)^{(0)}+\frac{D}{2}(\phi)^{(0)^{2}}
$$

A equaçäo $(6.25)$ pode ser reescrita como

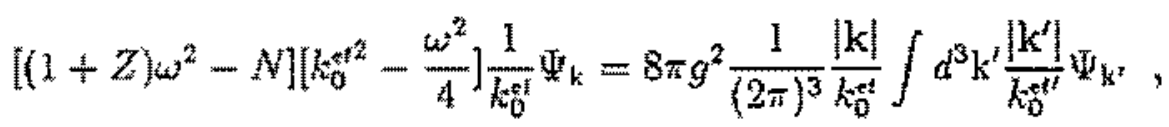

onde

$$
k_{0}^{+1}=\left(k^{2}+M^{2}\right)^{1 / 2}
$$

Devemos observar que o termo potencial da equaçào do tipo Schrötinger (6.27) é separável, podendo ser escrito como

$$
\begin{aligned}
\langle\mathrm{k}|l| \mathrm{k}\rangle & =v(\mathrm{k}) v^{*}\left(\mathrm{k}^{\prime}\right) \\
& =8 \pi g^{2} \frac{1}{(2 m)^{3}} h(\mathrm{k}) h\left(\mathrm{k}^{3}\right)
\end{aligned}
$$

onde

$$
h(k)=\frac{|\mathrm{k}|}{k_{0}^{k+1}}
$$

A solução da equaçào de Schrơdinger com potencial separável é conhecida da Teoria de Espalhamento [37]. onde a equaça de Lipppman-Schwinger pode ser resolvida de forma 
fechada. Em analogia com a Teoria de Espalhamento, a solução geral para a equação (6.27) tem duas componentes. A primeira corresponde a solução trivial do problema quando $g=0$. A segunda componente da soluçăo da amplitude $\Phi_{\mathrm{k}}$ é a parte nâa trivial, que contém a influència do termo potencial. Esta componente corresponde ao termo de onda espalhada na Teoria de Espalhamento.

Portanto, a soluçăo da equaçẫo (6.27) pode ser escrita como uma equação integral na forma de uma equação de Lippmann-Schwinger,

$$
\begin{aligned}
& \frac{|\mathrm{k}|}{k_{0}} \Psi(\mathrm{k}, \mathrm{q} ; w)=\alpha \delta(\mathrm{q}-\mathrm{k})+
\end{aligned}
$$

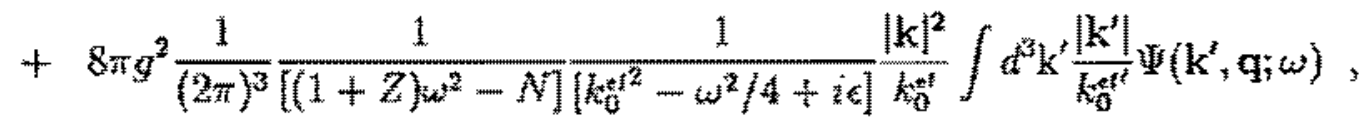

onde q é o momento relativo de dois quasi-fếrmions incidentes e $\alpha$ é um fator de fase inicial do sistema. Escolhemos para a soluçâo da equaçăo (6.27) a condição de contorno de ondas emergentes $(+i \epsilon)$. Observamos que agora devemos interpretar $\Psi_{\mathrm{k}}$ como uma função de onda de espalhamento dos dois quasi-férmions provenientes da flutuaçào do vácuo, enquanto $\omega$ é o espectro de excitação do vácuo gaussiano cono veremos adiante.

Para resolvermos a equaça integral (6.29), integramos en $k$ e fazemos a mudança $\mathrm{k} \leftrightarrow \mathrm{k}^{z}$ no termo potencial. Obtemos entă,

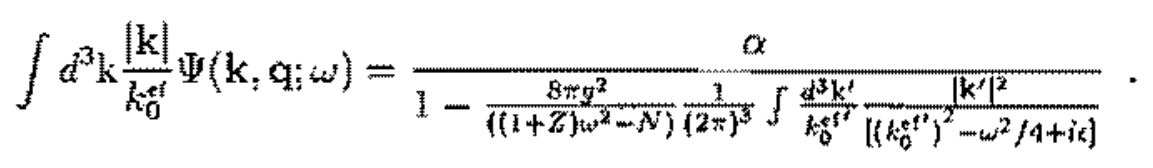

Substituindo este resultado na equaçăo $(6.29)$, temos a soluçăo para $\Psi_{k}$

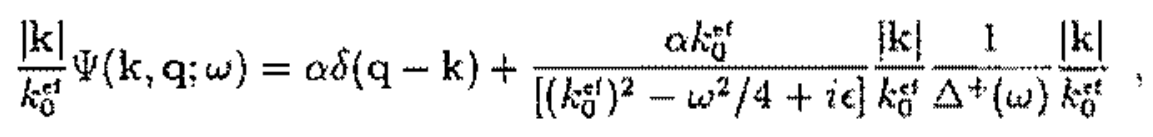

onde

$$
\Delta^{+}(\omega)=(2 \pi)^{3} \frac{\left[(1+Z) \omega^{2}-N\right]}{8 \pi g^{2}}-\int d^{3} k^{3} \frac{1}{\left[\left(k_{0}^{z^{\prime}}\right)^{2}-\omega^{2} / 4+i k\right]} \frac{\left|\mathbf{k}^{\prime}\right|^{2}}{k_{0}^{t^{\prime}}}
$$

Substituindo a solução geral (6.31). $\Psi_{\mathrm{k}}$, na equação (6.25). obtemos a frequência w de petuenas oscilações do emparelhamento em torno do equilibrio para o plasma escalar 


$$
w=2 q_{0}^{* 4}=2\left(q^{2}+M^{2}\right)^{1 / 2}
$$

onde $\mathrm{q}$ é o momento relativo de dois quasi-férmions incidentes e a frequência $\omega$ pode ser reinterpretada como a energia de centro de massa de dois quasi-férmions espalhados, ou ainda, o espectro de excitação do vácuo.

\subsection{Estados Ligados de Quasi-Férmions}

Nesta seçào estudaremos a possibilidade de existência de estados ligados de quasi-férmions (provenientes da flutuação do vácuo) no regime de pequenas oscilações em torno do equilibrio, a partir da analogia com a Teoria de Espalhamento. Da Teoria de Espalhamento temos para a matriz de transição $T$, no caso de potencial separável, a seguinte forma [37]

$$
r\left(k, k^{\prime} ; \omega\right) \propto h\left(k^{\prime}\right) \frac{1}{\Delta^{+}(\omega)} h(\mathbf{k})
$$

onde $h(\mathbf{k})$ é dado em $(6.28)$ e $\Delta^{+}(\omega)$ em $(6,32)$. Como estamos interessados na possibjlidade de existência de estados ligados de quasi-férmions, devemos estudar os polos da matriz de transição $T$ que correspondern aos zeros de $\Delta^{+}(\omega)$. Observamos portanto que a condição para existência de estados ligados depende da energia relativa $\omega$ dos quasjférmions incidentes. Ou seja, dada a energia relativa $\omega$, temos que verificar a existencia de $\Delta^{+}(\omega)$ nulo, o que corresponderia a um estado ligado. Se $\Delta^{+}(\omega)$ não é nulo, significa que os dois quasi-férmions sảo espallhados no contínuo.

A partir de (6.32) verificamos que $\Delta^{+}(\omega)$ contền divergências que säo canceladas pelos contratermos en $\left[(1+Z) \omega^{2}-N\right]$. Substituindo os contratermos $\delta z^{2}, C$ e $D$ dados em $(5.44-5.47)$ en $N$ dado pela equaçăo $(6.26)$ e escolnendo $Z$ da forma

$$
Z=4 \pi g^{2} L(m)
$$

obtemos

$$
\Delta^{+}(\omega)=\frac{1}{8 \pi g^{2}}\left[\left(\mathbf{i}+4 \pi g^{2} L(m)\right) \omega^{2}-\mu^{2}+16 \pi g^{2} G(0)-24 \pi g^{2} M^{2} L(m)\right]-I_{\omega},
$$

onde $I_{\omega}$ e a integral divergente

$$
I_{\omega}=\frac{4 \pi}{(2 \pi)^{3}} \int_{0}^{\Lambda} d k \frac{k^{2}}{\left(k^{2}+M^{2}\right)^{1 / 2}} \frac{1}{\left(k^{2}+M^{2}-\omega^{2} / 4\right)} .
$$


Temos agora que analisar a integral $I_{\omega}$. Verificarnos que para $0 \leq \omega<2 M$ năo há polos no eixo real, enquanto no caso em que $\omega \geq 2 M$ a integral $l_{\text {: }}$ apresenta polos no eixo real.

i) $0 \leq w<2 M$. Neste caso temos (vide apêndice C)

$$
\begin{aligned}
I_{\omega} & =\frac{1}{2 \pi^{2}}\left(\frac{\Lambda^{2}}{2}+\frac{M^{2}}{4}+\left(\frac{\omega^{2}}{4}-\frac{3 M^{2}}{2}\right) \ln \left(\frac{2 N}{M}\right)\right. \\
& \left.+\frac{\left(4 M^{2}-\omega^{2}\right)^{3 / 2}}{4 \omega} \arctan \left[\frac{\omega}{\left(4 M^{2}-w^{2}\right)^{1 / 2}}\right]\right\}
\end{aligned}
$$

Substituindo este resultado na equaçăo $(6.35)$, obtemos

$$
\Delta^{+}(\omega)=F(\omega)-\frac{\pi \mu^{2}}{g^{2} m^{2}}
$$

onde

$$
\begin{aligned}
F(\omega) & =\frac{\pi \omega^{2}}{g^{2} m^{2}}+\left[\left(\frac{\omega}{m}\right)^{2}-6\left(1-\frac{\left.g\langle\phi\rangle\right|_{\mathrm{eq}}}{m}\right)^{2}\right] \ln \left(1-\frac{\left.g\langle\phi\rangle\right|_{\mathrm{eq}}}{m}\right)-\left(1-\frac{\left.g(\phi\rangle\right|_{\mathrm{eq}}}{m}\right)^{2} \\
& -\frac{m}{\omega}\left[4\left(1-\frac{\left.g(\phi)\right|_{\mathrm{eq}}}{m}\right)^{2}-\left(\frac{\omega}{m}\right)^{2}\right]^{3 / 2} \arctan \left[\frac{\omega / m}{\left[\left(1-\left.g(\phi)\right|_{\mathrm{eq}} / m\right)^{2}-(\omega / m)^{2}\right]^{1 / 2}}\right]
\end{aligned}
$$

Neste ponto devemos observat que substituindo $(6,38-6.39)$ em (6.31) temos uma solução analítica renormalizada para a dinâmica de pequenas oscilaçóes em torno da situação de equilibrio do vácuo do plasma escalar quando $0 \leq \omega<2 M$.

Procuremos em seguida os zeros de $\mathrm{A}^{+}(\mathrm{t}, \mathrm{t})$. Para um dado valor da constante de

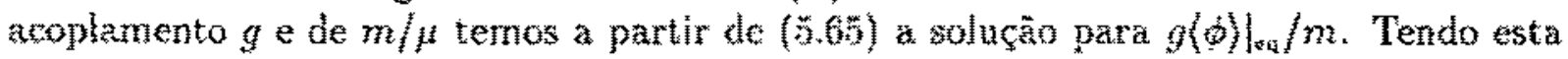
solução, substitumos na equaç̆o (6.39) e obternos a tunção $F(\omega)$. Nas Figuras abaixo mostramos o comportamento da funçäo $F(t ;)$ e $\pi h^{2} / g^{2} m^{2}$ para $m / n=1 / 2$ e 1 com constante de acoplamento $g^{2}=\pi / 4$ e $5 / 2$. Para $m / \mu=1$ temos estado ligado de dois quasi-férmions. enquanto que para m/ $t=1 / 2$ näo ha estado ligado. 
$m u=1 \mathrm{eg}^{2}=\mathrm{g}$

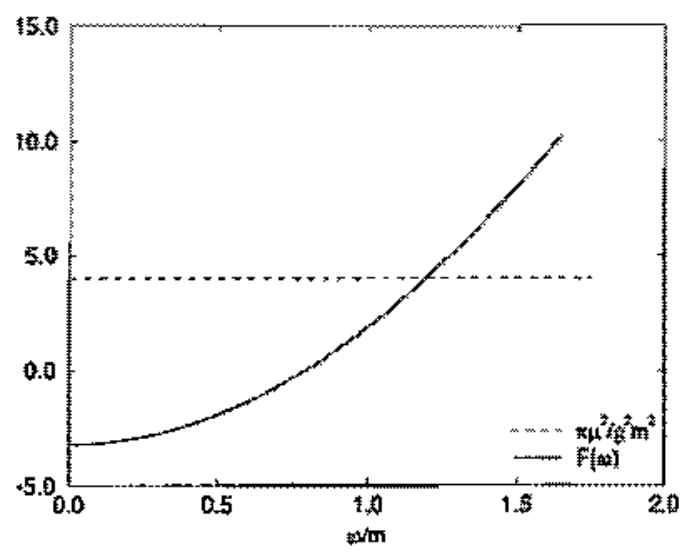

$m / n=1+g^{2}=5 \pi / 2$

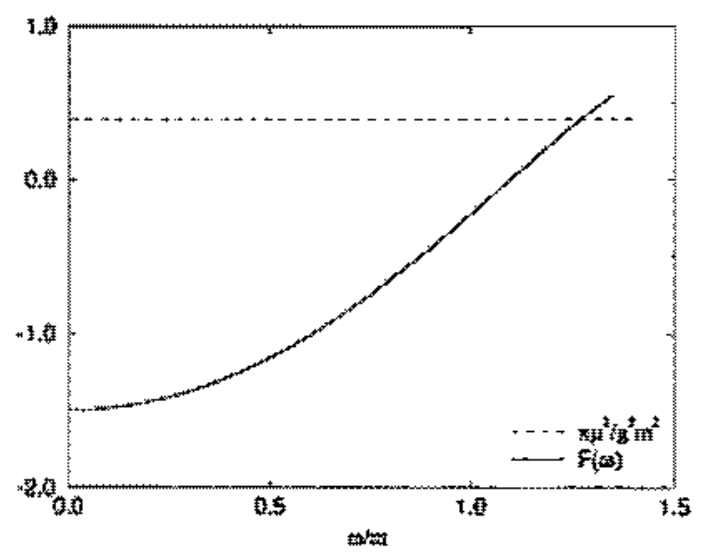

Figura 6.1: Função $F(\omega)$ e $\pi \mu^{2} / g^{2} m^{2}$ para $m / \mu=1$ e $g^{2}=\pi / 4,5 \pi / 2$. Para $g^{2}=\pi / 4$ a energia do estado ligado de quasi-férmions na escala de massa $m$ é $\omega / m=1,19$. Para $g^{2}=5 \pi / 2$ a energia do estado ligado é $\omega / m=1.27$.
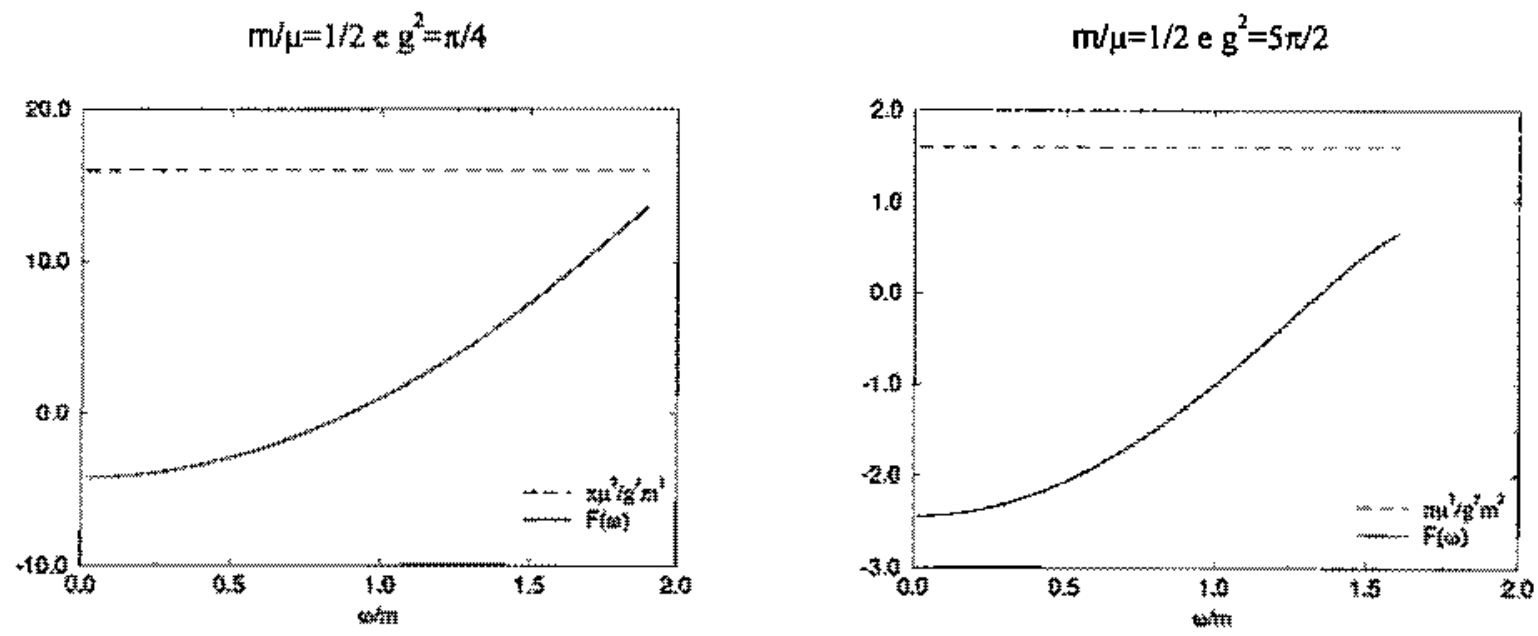

Figura 6.2: Funçầ $F(w)$ e $\pi \mu^{2} / g^{2} m^{2}$ para $m / \mu=1 / 2$ e $g^{2}=\pi / 4,5 \pi / 2$. Neste caso näo há estado ligado. 
Verifcames na Figura 6.1 que quanto maior a constante de acoplamento, maior é a energia do estado ligado de dois quasi-férmions, como era esperado. Verificarnos ainda pelas Figuras 6.1 e 6.2 que a existencia de estados ligados de dois quasi-férmions está relacionada com a massa $\mu$. Sendo $\mu$ a rnassa do potencial de Yukawa, à medida que $\mu$ aumenta, o alcance do potencial de Yukawa diminui. Isto explica o fato de não termos estado ligado para $m / \mu=1 / 2$.

ii) $\omega \geq 2 M$. Neste caso a integral $I_{\omega} \operatorname{tem}$ uma singularidade em $k=\sqrt{\omega^{2} / 4-M^{2}}$. Utilizando-se a Teoria de Residuos obtemos

$$
I_{\omega}=V P \int_{0}^{4} d \mathrm{k} \frac{k^{4}}{\left(\mathrm{k}^{2}+M^{2}\right)^{1 / 2}}\left[k^{2}-\left(\omega^{2} / 4-M^{2}\right)\right]+i \pi \frac{\omega^{2}}{8}\left[1-\frac{4 M^{2}}{\omega^{2}}\right]
$$

onde VP é o valor principal de Cauchy da integral $l_{w}$. A partir da tabela de integrais do apendice $\mathrm{C}_{3}$ calculamos o VP de Cauchy de $I_{w}$, obtendo

$$
\begin{aligned}
I_{w} & =\frac{1}{2 \pi^{2}}\left\{\frac{A^{2}}{2}+\frac{\left(6 M^{2}-w^{2}\right)}{4}\left[-\ln 2+\ln \left(\frac{M}{N}\right)\right]\right. \\
& \left.-\frac{w^{2}}{8}\left(1-\frac{4 M^{2}}{w^{2}}\right)^{3 / 2} \ln \left[\frac{w+\left(\omega^{2}-4 M^{2}\right)^{1 / 2}}{\omega-\left(w^{2}-4 M^{2}\right)^{1 / 2}}\right]+\frac{2 \pi}{8 \omega}\left(w^{2}-4 M^{2}\right)^{3 / 2}\right\}
\end{aligned}
$$

Substituindo este resultado na equação $(6.35)$ temos

$$
\begin{aligned}
\Delta^{+}(\omega) & =\frac{1}{S \pi^{2}}\left\{\left(\omega^{2}-6 M^{2}\right) \ln \left(\frac{M}{m}\right)-\frac{\mu^{2} \pi}{g^{2}}+\frac{\pi \omega^{2}}{g^{2}}\right\} \\
& \left.-\frac{1}{\omega}\left(\omega^{2}-4 M^{2}\right)^{3 / 2} \ln \left[\frac{\omega+\left(\omega^{2}-4 M^{2}\right)^{1 / 2}}{\omega+\left(\omega^{2}-4 M^{2}\right)^{1 / 2}}\right]+\frac{i \pi}{2 \omega}\left(\omega^{2}-4 h^{2}\right)^{3 / 2}\right\}
\end{aligned}
$$

Verificamos a partir de $(6.42)$ que $\Delta^{+}(\omega)$ nao se anula para $\omega>2 M$. Ou seja, nào há estados ligados de dois quasi-férmions se a energia relativa incidente destes for maior que $2 M$. 


\section{Capítulo 7}

\section{Conclusões e Perspectivas}

Neste trabalho estudamos a extensão da aproximaçâo de campo médio, dada una condiçâo inicial, para a evolução temporal de um sistema composto de férmions e bósons que interagem. Para isto usamos uma técrica de projeçäo dependente do tempo através da qual obtemos equaçóes de movimento do tipo cinético para o conjunto de variáveis dinâmicas de um corpo. Este formalismo possibilita incluir correlaçòes dinâmicas às equaçöes de campo médio, além de permitir o cálculo com matéria e com temperatura. Na primeira parte do trabalho aplicamos a técnica para um sistema descrito pelo modelo de Jaynes-Cummings e na segunda estudamos o modelo do plasma escalar relativistico.

No capítulo 3 obtivemos as equaçôes que descrevem a dinâmica na aproximaçăa com termos de correlaçầo para o modelo de Jaynes-Cummings. A evolução temporal da inversão atômica e da polarização intrínsica obtidas nesta aproximaçào foram comparadas com a soluçăo exata, mostrando que a inclusão dos termos de correlaçâo săo fundarnentais para gerar o amortecimento das oscilaçôes de Rabi resultante da correlaçào entre os dois subsistemas.

No capitulo 4 adaptamos o formalismo aplicado ao modelo de Jaynes-Cummings ao estudo de sistemas relativísticos. Apresentamos assim, um tratamento nầo perturbativo para o problema da condição inicial na Teoria Quântica de Campos para um sistema de férmions interagindo com bósons.

No capitulo b obtivemos as equaçôes cinéticas que descrevem a dinâmica efetiva das variáveis de um corpo para o plasma escalar relativístico na aproximaçấo de campo médio. A partir das soluçōes estacionárias para o vácuo das equaçöes cinéticas, renormalizamos a teoria incluindo contratermos. Verificamos neste processo de renomalização que o modelo do plasma escalar relativistico não pernite dinâmica de emparelhamento para as variàveis bosônicas. Enfim, estudamos o comportarnento da energia do vácuo gaussiano sleste modelo em função do condensado para diferentes valores da constante de acoplamento 
No capitulo 6 figemos uma aplicaçäo das equaçoes cinéticas obtidas no capitulo Estudamos a dinämica efetiva de pequenas oscilaçôes em torno da solução extacionária. Resolvemos estas equaçós analiticamente através de uma anaiogia com a teoria de espalhamento, obtendo as amplitudes de oscilação do emparelhamento e suas respectivas frequêneias. Desta analogia reinterpretamos o problema de pequenas oscilaçỏes do emparelhamento como um problema de espalhamento de dois quasi-férmions gerados da flututuçăo do vícuo. Finalmente, estudamos a condição de existência de estados ligados de quasi-férmions (provenientes da flutuação do vắcuo) neste regime.

Como perspectiva de futuros trabalhos, devemos ressaltar que todos os cálculos do plasma escalar relativístico foram feitos para o vácuo do sistema $\left(\nu_{k, s}^{(1)}=\nu_{k, s}^{(2)}=0\right)$. Dando continuidade a este trabalho, o primeiro passo seria estudar as equaçōes com matéria, o que introduz uma dependencia com relaçäo a densidade. Este tipo de cálculo consiste em tomar para $\mathcal{F}_{0}^{F}$ ocupaçöes nẫo rulas para estados de energia positiva até o momento de Fermi $k_{F}$ e ocupaçöes nulas acima de $k_{\mathrm{F}}$.

Outra extensăo imediata deste trabalho e incluir termos de correlação no estudo da dinâmica efetiva do plasma escalar relativistico como fó feito no modelo de JaynesCummings. 


\section{Apêndice A}

\section{Cálculo de traços}

Neste apendice mostramos alguns traços utilizados em nosso cálculo. Começamos calculando tracos das densidades bosônicas

1) $\mathcal{T}_{\mathrm{w}}\left(\beta_{a} \beta_{t} \mathcal{F}_{0}\right)$

$$
\begin{aligned}
& \operatorname{Tr}_{\mathrm{BF}}\left(\beta_{a}^{\dagger} \beta_{b} \mathcal{F}_{0}\right)=\operatorname{Tr}_{\mathrm{B}}\left(\beta_{a}^{\dagger} \beta_{b} \mathcal{F}_{0}^{\mathrm{B}} \mathcal{F}_{0}^{z}\right)
\end{aligned}
$$

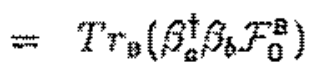

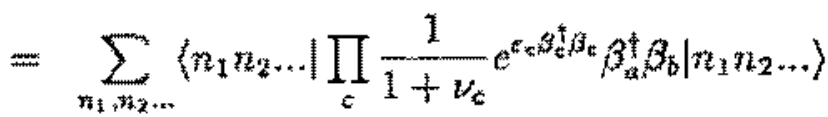

Para $a \neq b$ o traço é nulo e para $a=b$ obtemos

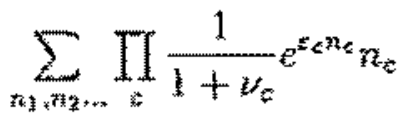

que pode ser reescrito como

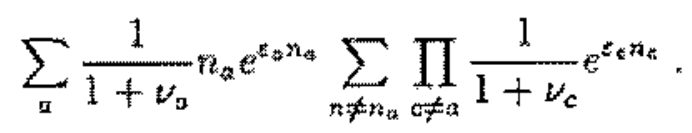

Utilizando a relaçâo 


$$
\sum_{n_{e}} e^{z_{e} n_{e}}=\frac{1}{1-e^{z_{c}}}
$$

e chamando

$$
e^{\epsilon_{c}}=\frac{\nu_{c}}{1+\nu_{0}}
$$

a parte que não depende de a é

$$
\sum_{n \neq n_{a}} \prod_{c \neq u} \frac{1}{1+\nu_{c}} \frac{1}{1-e^{z_{c}}}=1
$$

Logo, o traço é dado por

$$
\sum_{n_{a}} \frac{1}{1+\nu_{a}} n_{a} e^{e_{o} n_{a}}=\sum_{n_{a}} \frac{1}{1+\nu_{a}} \nu_{a x}\left(1+\nu_{a}\right)=\nu_{a}
$$

e portanto,

$$
\operatorname{Tr}_{\mathrm{BF}}\left(\beta_{a}^{\dagger} \beta_{b} \mathcal{F}_{0}\right)=\nu_{a} \delta_{a b}
$$

2) $\operatorname{Tr}_{\mathrm{BR}}\left(\beta_{\mathrm{a}}^{\dagger} \beta_{b}^{\dagger} \beta_{\mathrm{c}} \beta_{\mathrm{d}} \mathcal{F}_{0}\right)$

Temos três casos no qual este traço é diferente de zero:

i) $a=b=c=4$. Neste caso temos

$$
\operatorname{Tr}_{\mathrm{BF}}\left(\beta_{\mathrm{a}}^{\dagger} \beta_{a}^{\dagger} \beta_{a} \beta_{a} \mathcal{F}_{0}\right)=\operatorname{Tr}_{\mathrm{BF}}\left(\beta_{a}^{\dagger} \beta_{a} \beta_{a}^{\dagger} \beta_{a} \mathcal{F}_{0}\right)-\operatorname{Tr}_{\mathrm{gF}}\left(\beta_{a}^{\dagger} \beta_{a} \mathcal{F}_{0}\right)
$$

onde $T_{\mathrm{tar}}\left(\beta_{a} \beta_{\mathrm{B}} \mathcal{F}_{0}\right)=w_{a}$. O primeiro termo é

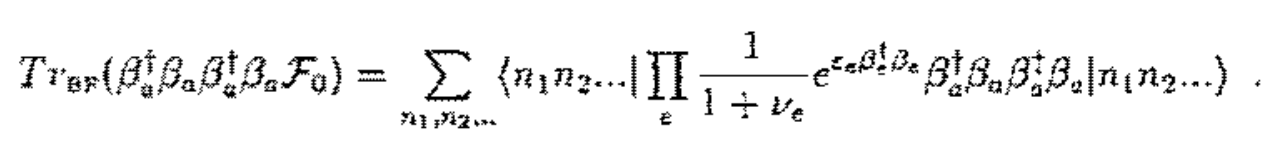

Usando a relação 


$$
\sum_{n_{a t}} e^{z_{a} n_{a}} n_{a}^{2}=v_{t}\left(1+t_{a}\right)\left(1+2 v_{a}\right),
$$

obtemos

$$
\operatorname{Tr}_{\mathrm{aF}}\left(\beta_{a}^{\dagger} \beta_{b}^{\dagger} \beta_{c} \beta_{d} \mathcal{F}_{0}\right)=2 \nu_{a}^{2}
$$

ii) $a=c$ e $b=d$. Neste caso escrevemos

$$
\beta_{a}^{\dagger} \beta_{b}^{\dagger} \beta_{c} \beta_{d}=\beta_{a}^{\dagger} \beta_{c} \beta_{b}^{\dagger} \beta_{d}-\beta_{a}^{\dagger} \beta_{d} \delta_{b c} .
$$

O segundo termo não contribui para o traço e o primeiro termo pode ser calculado de maneira análoga ao caso anterior. Obtemos para esse traço

$$
\operatorname{Tr}_{\mathrm{Br}}\left(\beta_{\mathrm{a}}^{\dagger} \beta_{b}^{\dagger} \beta_{c} \beta_{\mathrm{d}} \mathcal{F}_{0}\right)=\nu_{\alpha} \nu_{b} .
$$

iii) $a=b e c=d$. Fazendo o cálculo de maneira análoga ao caso anterior, obtemos

$$
\operatorname{Tr}_{B F}\left(\beta_{a}^{\dagger} \beta_{b}^{\dagger} \beta_{c} \beta_{d} \mathcal{F}_{0}\right)=\nu_{a} \nu_{b}\left(\delta_{\alpha t} \delta_{k d}+\delta_{a d} \delta_{b G}\right)
$$

Calculemos agora os traços das densidades fermionicas.

1) $\operatorname{Tr}_{\mathrm{p}}\left(\alpha_{\lambda}^{\dagger} \alpha_{\lambda} \mathcal{F}_{0}\right)$

$$
\begin{aligned}
& \operatorname{Tr}_{\mathrm{BF}}\left(\alpha_{\lambda}^{\dagger} \alpha_{\lambda} \mathcal{F}_{0}\right)=\operatorname{Tr}_{\mathrm{BF}}\left(\alpha_{\lambda}^{\dagger} \alpha_{\lambda} \mathcal{F}_{0}^{\mathrm{B}} \mathcal{F}_{0}^{\mathrm{F}}\right) \\
& =\operatorname{Tr}_{\mathrm{p}}\left(\alpha_{\lambda}^{\dagger} \alpha_{\lambda} \mathcal{F}_{0}^{\mathrm{F}}\right) \\
& =\operatorname{Tr}\left\{\alpha_{\lambda}^{\dagger} \alpha_{\lambda}\left[p_{\lambda} \alpha_{\lambda}^{\dagger} \alpha_{\lambda}+\left(1-p_{\lambda}\right) \alpha_{\lambda} \alpha_{\lambda}\right] \times\right.
\end{aligned}
$$

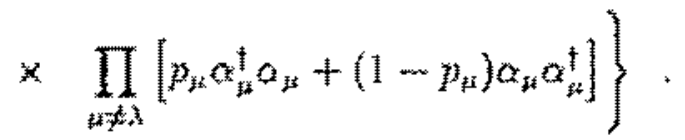

Observamos que

$$
\operatorname{Tr}_{\mathrm{BF}}\left\{\prod_{\mu \neq \lambda}\left[p_{\mu} \alpha_{\mu}^{\dagger} \alpha_{\mu}+\left(1-p_{\mu}\right) \alpha_{\mu} \alpha_{\mu}^{\dagger}\right]\right\}=1
$$


pois o cálculo deste traço consiste em somar todos os elementos diagonais de uma base no espaço de Fock, ou seja, na base dos autoestados do operador número $n_{\mu}$ com autovalores $p_{p}$. Portanto;

$$
\operatorname{Tr}_{B F}\left(\alpha_{\lambda}^{\dagger} \alpha_{\lambda} \mathcal{F}_{0}\right)=\operatorname{Tr}_{F}\left\{\alpha_{\lambda}^{\dagger} \alpha_{\lambda}\left[p_{\lambda} \alpha_{\lambda}^{\dagger} \alpha_{\lambda}+\left(1-p_{\lambda}\right) \alpha_{\lambda} \alpha_{\lambda}^{\dagger}\right]\right\}=p_{\lambda}
$$

onde usamos o fato de que $T T_{F}\left(\alpha_{\lambda}^{\dagger} \alpha_{\lambda} \alpha_{\lambda} \alpha_{\lambda}^{\dagger}\right)=0$, decorrente das regras de anticomutaçäo para os $\alpha_{\lambda}$.

2) $\operatorname{Tr}_{\mathrm{BF}}\left(\alpha_{k t}^{\dagger} \alpha_{\nu}^{\dagger} \alpha_{\lambda} \alpha_{p} F_{0}\right)$. Para densidades de dois corpos temos

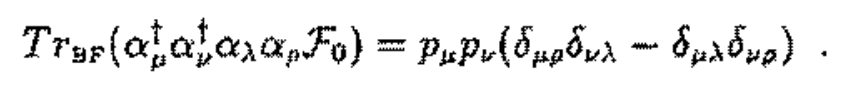




\section{Apêndice B}

\section{Aproximação para $\mathcal{F}^{\prime}(t)$ na Representação de Heisenberg}

Neste apêndice mostramos a aproximaçäo feita para $\mathcal{F}^{\prime}(t)$. Como vimos no capitulo 3 , cálculo de efeitos de correlaçăo das equaçöes dinâmicas envolvem traços do tipo

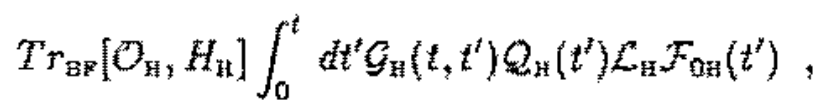

onde o indice $H$ indica a representaçäo de Heisenberg, $O$ corresponde aos operadores bosônticos e fermionnicos $\mathrm{e}$

$$
\mathcal{G}_{\mathrm{H}}\left(t, t^{\prime}\right)=T \exp \left\{i \int_{z^{z}}^{t} d \tau \mathcal{P}_{\mathrm{H}}(\tau) \mathcal{C}_{\mathrm{H}}(\tau)\right\}
$$

é o propagador na representação te Heisenberg.

Lembrando $q u O_{\mathrm{y}}=e^{i H} \mathrm{O}_{\mathrm{s}} \mathrm{e}^{-\mathrm{i} H t}$, podemos escrever o termo de correlação como

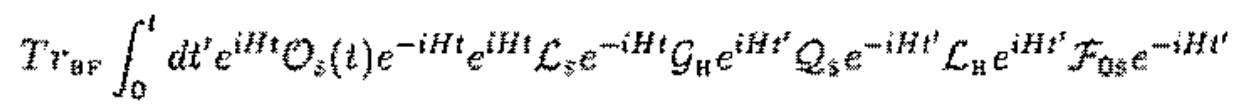

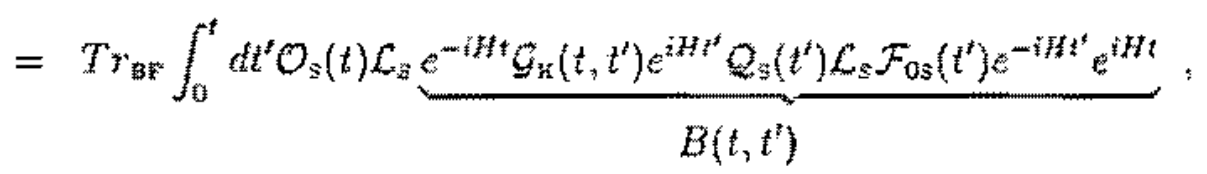

onde na última passagem usamos a propriedade éclica do traço. 
Examinemos $B\left(t, t^{*}\right)$ atravếs da equação

$$
B\left(t, t^{\prime}\right)=\mathcal{G}_{\mathrm{H}}\left(t, t^{\prime}\right) Q_{\mathrm{H}}\left(t^{\prime}\right) \mathcal{L}_{\mathrm{H}} \mathcal{F}_{\mathrm{OH}}\left(t^{3}\right)
$$

Derivando com relação jo tempo

$$
\begin{aligned}
\frac{d}{d t} B\left(t, t^{\prime}\right) & =i\left(-i H B\left(t, t^{\prime}\right)+i B\left(t, t^{\prime}\right) H\right)+ \\
& +i e^{-i H t_{i}} P_{\mathrm{H}} \mathcal{L}_{\mathrm{B}} \mathcal{G}_{\mathrm{B}}\left(t, t^{\prime}\right) e^{i H t^{t}} \mathcal{F}_{03}\left(t^{\prime}\right) e^{-i H t^{t}} e^{i H t} \\
& =\left(H B\left(t, t^{\prime}\right)-B\left(t, t^{\prime}\right) H\right)-e^{-i H t} \mathcal{P}_{\mathrm{s}} e^{i H t} e^{-i H t} \mathcal{L}_{\mathrm{H}} e^{i H t} B\left(t, t^{\prime}\right) \\
& =\mathcal{L}_{\mathrm{s}} B\left(t, t^{\prime}\right)-\mathcal{P}_{\mathrm{B}} \mathcal{L}_{\mathrm{S}} B\left(t, t^{\prime}\right) \\
& =Q_{\mathrm{B}} B\left(t, t^{\prime}\right)
\end{aligned}
$$

A solução da eq. (B.4) é dada por

$$
\begin{aligned}
& B\left(t, t^{t}\right)=T \exp \left\{-i \int_{t^{*}}^{t} d T \mathcal{Q}_{\mathcal{S}}(\tau) \mathcal{L}_{\mathrm{s}}\right\} \mathcal{Q}_{\mathrm{s}}\left(t^{t}\right) \mathcal{L}_{\mathrm{s}} \mathcal{F}_{6 s} \\
& =\mathcal{G}_{3}\left(t, t^{\prime}\right) \mathcal{Q}_{\mathrm{s}}\left(t^{t}\right) \mathcal{L}_{3} \mathcal{F}_{03} \text {. }
\end{aligned}
$$

Logo, na representacäo de Schrödinger temos a mesma expressäo para o termo de correlação

$$
\operatorname{Tr} \int_{0}^{t} d t^{\prime} \mathcal{O}_{s}(t) \mathcal{L}_{s} \mathcal{C}_{s}\left(t, t^{\prime}\right) Q_{s} \mathcal{C}_{s} \mathcal{F}_{d s}
$$

mas neste caso a funcào de Green é dada por

$$
\mathcal{G}_{s}\left(t, t^{t}\right)=T \exp \left\{-i \int_{i^{t}}^{t} d \tau Q_{s}(\tau) \mathcal{L}_{s}(\tau)\right\} .
$$


Seguindo Lin [33] implementamos na representaçâo de Heisenberg uma versão modificada da aproximaçã de majs baixa ordem da referência [26]. Neste trabalho, os autores mostraram uma maneira de aproximar o propagador $9\left(q_{1}, t^{\prime}\right)$ na representaçào de Sehrödinger. Eles expandem $\mathcal{G}\left(t, t^{t}\right)$ en séries de potências de um propagador más simples definido por

$$
G_{\mathrm{s}}\left(t, t^{\prime}\right)=T \exp \left\{-i \int_{t^{\prime}}^{t} d r \mathcal{L}_{0 s}(\tau)\right\}
$$

onde dividimos

$$
H=H_{0}+H_{1}
$$

e definimos o operador de Liouville

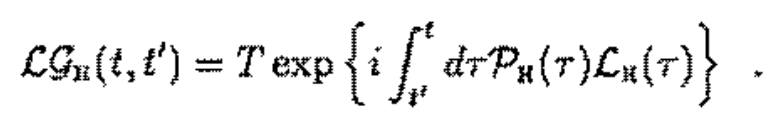

Os primeiros termos da série säo

$$
\begin{aligned}
& G_{\mathbb{s}}^{(\omega)}\left(t, t^{\prime}\right)=G_{\mathrm{s}}\left(t, t^{\prime}\right) \\
& \mathcal{G}_{S}^{(1)}\left(t, t^{\prime}\right)=\mathcal{G}_{0 s}\left(t, t^{\prime}\right)-i \int_{t^{*}}^{t} d t^{n} \mathcal{G}_{05}\left(t^{\prime}, t h\right) \mathcal{Q}_{s}\left(t^{t}\right) \mathcal{L}_{1}\left(t^{\prime \prime}\right) G_{0 \mathrm{a}}\left(t^{*}, t^{t}\right)
\end{aligned}
$$

Na representaçăo de Heisenberg temos

$$
O_{\xi} \simeq e^{-i H_{0 t}} O_{H} e^{i H_{0} t}
$$

onde aproximamos a evoluça do operador por $H_{0} . A$ aproximaçäo de mais baisa orden dessa série para o traço $(\mathrm{B} .6)$ é 


$$
\begin{aligned}
& T_{r_{\mathrm{ar}}} \int_{0}^{t} d t_{1} \mathcal{O}_{\mathrm{s}}(t) \mathcal{L}_{\mathrm{s}}(t) \mathcal{G}_{\mathrm{s}}^{(0)}\left(t_{1} t_{1}\right) Q_{\mathrm{s}}\left(t_{1}\right) \mathcal{L}_{\mathrm{s}}\left(t_{1}\right) F_{\mathrm{OS}}\left(t_{1}\right) \\
& =\operatorname{Tr}_{r_{\mathrm{BF}}} \int_{0}^{t} d t_{1} \mathcal{O}_{\mathrm{B}}(t) \mathcal{L}_{\mathrm{H}}(t) e^{i x_{0} t} \mathcal{G}_{\mathrm{s}}^{(0)}\left(t, t_{1}\right) e^{-i H_{0} t_{1}} \mathcal{Q}_{\mathrm{H}}\left(t_{1}\right) \mathcal{L}_{\mathrm{B}}\left(t_{1}\right) F_{\mathrm{OH}}\left(t_{1}\right) e^{i H_{0} t_{t}} e^{-i H_{0} t}
\end{aligned}
$$

onde usamos a equaç̃̃o (B.12) além da invariância cicliça do traco. O próximo passo é definir

$$
A\left(t, t_{1}\right) \equiv e^{i H_{0} t} \mathcal{G}_{S}^{(0)}\left(t, t_{1}\right) e^{-i H_{0} t_{1}} \mathcal{Q}_{\mathrm{H}}\left(t_{1}\right) \mathcal{L}_{\mathrm{H}}\left(t_{1}\right) \mathcal{F}_{\mathrm{OH}}\left(t_{1}\right) e^{i H_{0} t_{1}} e^{-i H_{\mathrm{O}} t}
$$

Portanto,

$$
A\left(t_{1}, t_{1}\right)=Q_{1}\left(t_{1}\right) C_{1}\left(t_{1}\right) F_{0 H}\left(t_{1}\right)
$$

Usando (B.8) e derivando a eq.(B.14), temos

$$
i f\left(t, t_{1}\right)=0
$$

cuja solução com a condição inicial $(\mathrm{B} .15)$ é dada por

$$
A\left(t_{1} t_{1}\right)=Q_{A}\left(t_{1}\right) L_{\mathrm{H}}\left(t_{1}\right) \mathcal{F}_{0 \mathrm{H}}\left(t_{1}\right)
$$

Logo, a aproximação de ordem mais baixa para $\mathcal{G}_{H}\left(t, t_{1}\right)$ de $(B .1)$ é

$$
G_{n}\left(t, t_{1}\right)=I
$$

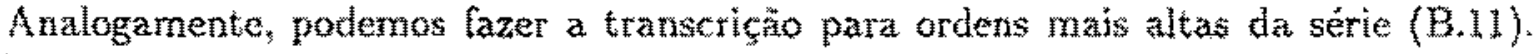
Enfim, a densidade pole ser escrita como tuma expansăo em $H_{0}$

$$
\begin{aligned}
\mathcal{F}^{\prime}(t) & =-i \int_{0}^{t} d t_{1} Q\left(t_{1}\right) \mathcal{L} \mathcal{F}_{0}\left(t_{1}\right) \\
& -\int_{0}^{t} d t_{1}\left(\int_{t_{1}}^{t} d t_{2} Q\left(t_{2}\right) \mathcal{L}_{1}\left(t_{2}\right)\right) Q\left(t_{1}\right) \mathcal{L Q}\left(t_{1} \mathcal{L}_{0} \mathcal{F}_{0}\left(t_{1}\right)+\ldots\right.
\end{aligned}
$$




\section{Apêndice C}

\section{Tabela de Integrais}

Escrevemos abaixo as integrais utilizadas neste trabalho.

$$
\begin{aligned}
& \int d x \frac{1}{\left(x^{2}+a^{2}\right)^{1 / 2}}=\ln \left[x+\left(x^{2}+a^{2}\right)^{1 / 2}\right] \\
& \int d x \frac{x^{2}}{\left(x^{2}+a^{2}\right)^{1 / 2}}=\frac{x\left(x^{2}+a^{2}\right)^{1 / 2}}{2}-\frac{a}{2} \ln \left[x+\left(x^{2}+a^{2}\right)^{1 / 2}\right] \\
& \int d x x^{2}\left(x^{2}+a^{2}\right)^{1 / 2}=\frac{x\left(x^{2}+a^{2}\right)^{3 / 2}}{4}-\frac{a^{2} x\left(x^{2}+a^{2}\right)^{1 / 2}}{8}-\frac{a^{4}}{8} \ln \left[x+\left(x^{2}+a^{2}\right)^{1 / 2}\right] \\
& \int d x \frac{x^{2}}{\left(x^{2}+a^{2}\right)^{3 / 2}}=-\frac{x}{\left(x^{2}+a^{2}\right)^{1 / 2}}+\ln \left[x+\left(x^{2}+a^{2}\right)^{1 / 2}\right]
\end{aligned}
$$




$$
\begin{aligned}
& \int d x \frac{x^{4}}{\left(x^{2}+b\right)^{1 / 2}\left(x^{2}+(x)\right)}= \\
& =\frac{x\left(b+x^{2}\right)^{1 / 2}}{2}-\frac{(b-2 a)}{2} \ln \left[x+\left(b+x^{2}\right)^{1 / 3}\right] \\
& -a\left(\frac{a}{2(a+b)}\right)^{1 / 2} \ln \left[\frac{x(a+b)^{1 / 2}+a^{1 / 2}\left(b+x^{2}\right)^{1 / 2}}{x(a+b)^{1 / 2}-a^{1 / 2}\left(b+x^{2}\right)^{1 / 2}}\right] \text { se } a-b>0 \\
& =\frac{x\left(x^{2}+b\right)^{1 / 2}}{2}-\frac{(b+2 a)}{2} \ln \left[x+\left(x^{2}+b\right)^{(1 / 2)}\right] \\
& +\frac{a^{3 / 2}}{(b-a)^{1 / 2}} \arctan \left[\frac{(b-a)^{1 / 2}}{b^{1 / 2}\left(a+x^{2}\right)^{1 / 2}}\right] \text { se } b-a>0
\end{aligned}
$$




\section{Bibliografia}

[1] F. Haake, em Springer Tracts in Modern Physics Vol.66, Springer-Verlag, Berlin, 1973.

[2] R. Zwanzig, en Lectures in Theoretical Physics Vol.3, Interscience, New York, 1961.

[3] C. Willis e R. Picard, Phys. Rev. A9, 1343 (1974).

[4] P. Bonche, S. Koonin e W. Negele, Phys. Rev. C13, 1226 (1976);

H. Reinhardt, Nucl. Phys. A.331, 353 (1979);

A. Kerman e S. Levit, Phys. Rev. C24, 1029 (1981).

[5] A. Kerman e T. Troudet, Ann. of Phys, 154, 456 (1984).

[6] C. Wong e H. Tang, Phys Rev. Lett. 40, 1070 (1978).

(7) H. Orland e R. Shaeffer, z. Phys. A290, 191 (1979);

S. Ayik, Z. Phys. A298, 83 (1980);

P Gragé, H. Weindenmüller e G. Wolschin, Ann. Phys. (N.Y.) 136, 190 (1981);

P. Buck e H. Feldmeier, Phys. Lett. B129, 172 (1983);

S. Wang e W. Cassing, Ann. Phys. (N.Y.) 159, 328 (1985).

[8] A.F.R. de Toledo Piza, em Time Dependent Hartree-Fock and Beyond, Lectures Notes in Physics Vol.171, Springer-Verlag, Berlin, 1982;

M.C. Nemes e A.F.R. de Toledo Piza, Phys. Rev. C27, 862 (1983);

M.C. Nemes e A.F.R. de Toledo Piza, Phys. Rev. C31, 613 (1985); M.C. Nemese A.F.R. de Toledo Piza, Phys. A.137, 367 (1986);

B. Carison, M.C. Nemes e A.F.R. de Toledo Piza, Nucl. Phys. A457, 261 (1986);

A.F.R. de Toledo Piza, Publicaçăo IFUSP/P-659 (1987). 
[9] Y. Nambu e G. Jona-Lasinio, Phys. Rev. 122, 345 (1961); idem 124, 246 (1961);

J. Walecka, Ann. Phys. (N.Y.) 83, 491 (1974);

S. Chang, Phys. Rev. D12, 1071 (1975);

S.A. Chin, Ann. Phys. (N.Y.) 108, 301 (1977);

R. Hakim, Riv. N. Cim., 1, 1 (1978);

L. Celenza e C. Shakin, Phys. Rev. C24, 2704 (1981);

J. Alonso, Phys. Rev. D31, 1315 (1985);

B. Serot e J. Walecka, em The relativistic Nuclear Many-Body Problem, Advances in Nuclear Physics - volume 16, Plenum Press, New York (1986);

B. Serot, Rep. Prog. Phys. 55, 1855 (1992);

R. Furnstahl, H.-B. Tang e B. Serot, Phys. Rev. C52, 1368 (1995).

[10] A. Kerman e D. Vautherin, Ann. Phys. (N.Y.) 192, 408 (1989).

[11] S. Coleman, R. Jackiw e H.D. Politzer , Phys. Rev. D10, 2491 (1974);

D. Gross e A. Neveu, Phys. Rev. D10, 3235 (1974).

[12] J. Alonso e R. Hakim, Phys. Rev. D29, 2690 (1984).

[13] A. Starobinsky, Phys. Lett. B91, 99 (1980);

A. Guth, Phys. Rev. D23, 347 (1981);

A. D. Linde, Particle Physics and Inflationary Cosmology, Harwood Academic Publishers (1990).

[14] O. Éboli, R. Jackiw e So-Yong Pi, Phys. Rev. D37, 3557 (1988);

M. Samiullah, O. Éboli e So-Yong Pi, Phys. Rev. D44, 2335 (1991);

D. Boyanovsky, H.J. de Vega e R. Holman, Phys. Rev. D49, 2769 (1994);

F. Braghin, C. Martin e D. Vautherin, Phys. Lett. B348, 343 (1995);

Érica Regina Takano. dissertação de mestrado IFUSP.-1992, Prof. Orientador O. Éboli.

[15] H.-Th. Else,M. Gyulassy e D. Vasak, Phys. Lett. B177, 402 (1986).

[16] R. Horsley e W. Shoenmaker, Nucl. Phys. B280. 716 (1987). 
[17] C. Miug Ko, Qi Li e R. Wang Phys. Rev. Lett. 59, 1084 (1987);

W. Cassing, G. Batko, B. Blättel, V. Koch, A. Lang, U. Mosel, K. Niita, K. Weber e G. Wolf, Nucl. Phys. A519, $357 \mathrm{f}$ (1990);

G. Baym, Ultrarelativistic Heavy-Ion Collisions, em Proceeding of IV International Summer School, la Rábida, Spain, Springer Verlag (1992).

[18] J. Providência, M. Ruivo e C. Souza, Phys. Rev. D36, 1882 (1987);

W. Cassing, A. Lang, S. Tèis e K. Weber, Nucl. Phys. A545, 123c (1992);

[19] P.L. Natti e A.F.R. de Toledo Piza, Phys. Rev. D54, 7867 (1995).

[20] P. Stevenson, B. Allès and R. Tarrach, Phys. Rev. D35, 2407 (1987);

A. Kovner and B. Rosenstein, Phys. Rev. D39, 2332 (1989).

[21] A. Kerman e C.-Yong Lin, Ann. Phys. (N.Y.) 241, 185 (1995).

[22] M. Ploszajczak, M. Rhodes-Brown, Phys. Rev. D33, 3686 (1986);

J. Alonso e A. Canyellas, Nucl. Phys. A526, 623 (1991).

[23] J. Alonso e R. Hakim, Phys. Rey. D38, 1780, (1988).

[24] S.J. Wang, W. Cassing, Nucl. Phys. A495, 371: (1989);

[25] C.Y. Lin e A.F.R. de Toledo Piza, Phys. Rev. D46, 742 (1992).

[26] P. Buck, H. Feldmeier e M.C. Nemes, Ann. Phys. (N.Y.) 185, 170 (1988).

[27] E.T. Jaynes and F.W. Cummings, Proc. IEEE 51, 89 (1963)

Pierre Meystre em Cavity Quantum Optics and the Quantum Measurement process, Progress in Optics, Vol. XXX, pg. 261. Edited by E. Wolf, Elsevier Publishers, 1992.

[28] G. Kalman, Phys. Rev. D9, 1656 (1974).

[29] E.R. Takano Natti e A.F.R. de Toledo Piza, Phys, A236, 321 (1997).

[30] E.R. Takano Natti e A.F.R. de Toledo Piza, em Iritinl-conditons problem for a relativistatic quantum sealar plasma. Publicaçào IFUSP/P-xxxx (1997), HEP-NET xxxx: Submetido a Phys. Rev. D.

[31] P. Ring e P. Schuck, The Many-Body Problem. Springer Verlag (1980). 
[32] J. des Cloiseaux, em Problème à N Corps, École d'été de Physique Théorique les Houches, Gordon and Breach (1967).

[33] C.Y. Lin e A.F.R. de Toledo Piza, Mod. Phys. Lett. A5, 1605 (1990).

[34] H.M. Nussenzveig, Introduction to Quantum Optics, Gordon and Breach Science Publishers (1973).

[35] L.C. Yong Tese de Doutoramento IFUSP (1991).

[36] D. Rowe, Nucl. Phys. 80, 209 (1966)

M.C. Nemes e A.F.R. de Toledo Piza, Z. Phys. A310, 339 (1983)

M.C. Nemes e A.F.R. de Toledo Piza, Phys. Rev. C31, 613 (1985).

[37] R. Newton, Scattering Theory of Waves and Particles, McGraw-Hill Book Company (1966).

[38] P.L. Natti A.F.R. de Toledo Piza, Phys. Rev. D55, 3403 (1997). 